\title{
RANDOM WALKS ON DISCRETE GROUPS OF POLYNOMIAL VOLUME GROWTH
}

\author{
By Georgios K. Alexopoulos \\ Université de Paris-Sud
}

\begin{abstract}
Let $\mu$ be a probability measure with finite support on a discrete group $\Gamma$ of polynomial volume growth. The main purpose of this paper is to study the asymptotic behavior of the convolution powers $\mu^{* n}$ of $\mu$. If $\mu$ is centered, then we prove upper and lower Gaussian estimates. We prove a central limit theorem and we give a generalization of the Berry-Esseen theorem. These results also extend to noncentered probability measures. We study the associated Riesz transform operators. The main tool is a parabolic Harnack inequality for centered probability measures which is proved by using ideas from homogenization theory and by adapting the method of Krylov and Safonov. This inequality implies that the positive $\mu$-harmonic functions are constant. Finally we give a characterization of the $\mu$-harmonic functions which grow polynomially.
\end{abstract}

1. Introduction and statement of the results. Let $\Gamma$ be a finitely generated discrete group of polynomial volume growth, let $\mu$ be a probability measure with finite support on $\Gamma$ and let $\mu^{* n}=\mu * \mu * \cdots * \mu$ be the $n$th convolution power of $\mu$.

The main purpose of this paper is to study the asymptotic behavior of $\mu^{* n}$. We obtain generalizations of certain results concerning the lattice valued distributions in $\mathbb{R}^{n}$ (cf. [20, 34]). We shall also extend certain results of [2, 15, 23] to nonsymmetric probability measures.

The measure $\mu$ can be either centered or not centered. It turns out that if $\mu$ is not centered, then we can conjugate $\mu$ by a multiplicative function and obtain another centered measure $\mu^{\prime}$. So it is enough to consider only centered measures.

According to a famous theorem of Gromov [22], $\Gamma$ is a finite extension of a nilpotent subgroup $\Gamma_{N} \triangleleft \Gamma$. By considering a subgroup of $\Gamma_{N}$ if necessary we can assume that $\Gamma_{N}$ can be embedded as a lattice in a simply connected nilpotent Lie group $N$. We can associate with $\mu$ a centered left invariant sub-Laplacian on $N$ denoted by $L_{H \mu} . L_{H \mu}$ is defined by a formula similar to the one we have in classical homogenization theory (cf. $[13,26]$ ).

Let $p_{t}^{H \mu}(x, y)$ be the heat kernel $L_{H \mu}$ [i.e., of the fundametal solution of the associated heat equation $\left(\frac{\partial}{\partial t}+L\right) u=0$ ]. Comparing $\mu^{* n}$ with $p_{t}^{H \mu}(x, y)$, we can obtain information on the distribution of the mass of $\mu^{* n}$. Using this information, together with a result of Varopoulos [49] which gives a uniform upper bound

Received September 1999; revised August 2001.

AMS 2000 subject classifications. 43A80, 60J15, 60B15, 20F65, 22E25, $22 \mathrm{E} 30$.

Key words and phrases. Random walk, group, convolution, Harnack inequality, heat kernel. 
on $\mu^{* n}$, it is possible to adapt the method of Krylov and Safonov (cf. [29, 30, 41]) and obtain a parabolic Harnack inequality.

Applying this inequality we can obtain upper and lower Gaussian bounds for $\mu^{* n}$. We can also prove, adapting some ideas of Bergström (cf. $\left.[14,41]\right)$, that as $n \rightarrow \infty$, the values of $\mu^{* n}$ approach the values of $p_{t}^{H \mu}(x, y)$ with uniform speed $1 / n^{\gamma / 2}$, for some $\gamma \in(0,1]$. Of course by the classical Berry-Esseen theorem (cf. $[20,34]$ ), the optimal rate of convergence is $1 / \sqrt{n}$. This is proved with the same method a posteriori, once we have the appropriate estimate for the space differences of $\mu^{* n}$.

The Berry-Esseen estimate implies that, on large balls, the $\mu$-harmonic functions look like $L_{H \mu}$-harmonic functions. Using this observation, we can adapt some ideas of Avellaneda and Lin [3, 4, 8-10] and prove a Taylor formula for the $\mu$-harmonic functions. This formula gives Harnack inequalities for the time and space differences of $\mu^{* n}$. It can also be used to obtain a caracterization of the $\mu$-harmonic functions which grow polynomially.

Finally, we prove Berry-Esseen estimates for the time and space differences of $\mu^{* n}$. We apply these estimates to study the associated Riesz transform operators.

1.1. Centered probability measures. The group $\Gamma /[\Gamma, \Gamma]$ is finitely generated and Abelian and hence it can be written as a direct product $\mathbb{Z}^{k} \times A$, where $A$ is finite and Abelian. Let $\pi$ be the canonical projection $\pi: G \rightarrow \mathbb{Z}^{k}$ and let $H=\operatorname{Ker}(\pi)$.

Let $\mu$ be a probability measure on $\Gamma$ whose support is finite and generates $\Gamma$. We say that $\mu$ is centered if the first order moments of its projection $\pi(\mu)$ on $\mathbb{Z}^{k}$ vanish, that is, if $\sum_{x \in \Gamma} \pi(x)_{i} \mu(x)=0,1 \leq i \leq k$, where $y_{i}$ is the $i$ th coordinate of the element $y=\left(y_{1}, \ldots, y_{k}\right) \in \mathbb{Z}^{k}, 1 \leq i \leq k$.

1.2. The passage from a noncentered to a centered probability measure. We say that $\chi: \Gamma \rightarrow \mathbb{R}^{+}$is multiplicative if $\chi(x y)=\chi(x) \chi(y), x, y \in \Gamma$. Note that then $\chi$ can be written as

$$
\chi=\phi \circ \pi \quad \text { with } \phi(x)=e^{\langle b, x\rangle},
$$

where $\pi$ is the quotient map $\pi: G \rightarrow \mathbb{Z}^{k} \cong G / H$ and where $\langle b, x\rangle=b_{1} x_{1}+\cdots+$ $b_{k} x_{k}$ for $b=\left(b_{1}, \ldots, b_{k}\right), x=\left(x_{1}, \ldots, x_{k}\right) \in \mathbb{R}^{k}$.

Let $\mu$ be a probability measure on $\Gamma$ whose support is finite and generates $\Gamma$. We have the following well-known lemma.

LEMMA 1.1. If $\mu$ is not centered, there are a multiplicative function $\chi$, a constant $\beta_{\mu}>0$ and another centered probability measure $\mu^{\prime}$ on $\Gamma$ such that

$$
\mu(x)=e^{-\beta_{\mu}} \mu^{\prime}(x) \chi(x), \quad x \in \Gamma .
$$


Note that (1.1) implies that

$$
\mu^{* n}(x)=e^{-\beta_{\mu} n} \mu^{\prime * n}(x) \chi(x), \quad x \in \Gamma .
$$

PROOF. Let $\pi(\mu)$ be the image of $\mu$ under the quotient map $\pi: \Gamma \rightarrow \mathbb{Z}^{k} \cong$ $G / H$ and let us consider the function

$$
F(a)=\sum_{x \in \mathbb{Z}^{k}} \pi(\mu)(x) e^{\langle a, x\rangle},
$$

where $\langle a, x\rangle=a_{1} x_{1}+\cdots+a_{k} x_{k}$ for $a=\left(a_{1}, \ldots, a_{k}\right), x=\left(x_{1}, \ldots, x_{k}\right) \in \mathbb{R}^{k}$.

We observe that $F$ is a positive smooth function on $\mathbb{R}^{k}$ and that $F(a) \rightarrow \infty$ as $|a| \rightarrow \infty$. So $F$ attains its minimum $b_{\varphi}=\min \left\{F(a): a \in \mathbb{R}^{k}\right\}$ at some point $a_{0} \in \mathbb{R}^{k}$. Also $F(0)=1$ and, since $\varphi$ is not centered, $\nabla F(0) \neq 0$. Hence $b_{\varphi}=$ $F\left(a_{0}\right)<1$ and $a_{0} \neq 0$. The lemma follows by taking

$$
\beta_{\mu}=-\log b_{\mu}, \quad \chi(x)=e^{-\left\langle a_{0}, \pi(x)\right\rangle}
$$

and

$$
\mu^{\prime}(x)=\frac{1}{b_{\mu}} \mu(x) e^{\left\langle a_{0}, \pi(x)\right\rangle}, \quad x \in \Gamma .
$$

The fact that $\nabla F\left(a_{0}\right)=0$ implies that $\mu^{\prime}$ is indeed a centered probability measure.

1.3. The geometry of $\Gamma$ and the sub-Laplacian $L_{H \mu}$. Let us fix a subset $U$ of $\Gamma$ such that the following hold:

1. $U$ is finite and generates $\Gamma$;

2. $e \in U$ ( $e$ is the identity element of $\Gamma)$;

3. $U$ is symmetric; that is, $x \in U$ if and only if $x^{-1} \in U$.

Let $U^{n}=\left\{x_{1} x_{2} \cdots x_{n}: x_{i} \in U, 1 \leq i \leq n\right\}$ and set

$$
|x|_{\Gamma}=\min \left\{n: x \in U^{n}\right\} .
$$

Also, let $|A|$ denote the number of elements of $A \subseteq \Gamma$.

In this article we assume that $\Gamma$ has polynomial volume growth, that is, that there are constants $c>0$ and $A \in \mathbb{N}$ such that $\left|U^{n}\right| \leq c n^{A}$, for all $n \in \mathbb{N}$. By a theorem of Gromov [22], this assumption implies that there is a nilpotent subgroup $\Gamma_{N} \triangleleft \Gamma$ such that $\left|\Gamma / \Gamma_{N}\right|<\infty$. Hence, by a theorem of Bass [11], there is an integer $D \geq 0$ such that

$$
\frac{1}{c} n^{D} \leq\left|U^{n}\right| \leq c n^{D}, \quad n \in \mathbb{N} .
$$

We call $D$ the homogeneous dimension of $\Gamma$. Note that $D$ does not depend on the choice of $U$. 
Let $\pi$ the quotient map $\pi: \Gamma \rightarrow \Gamma / \Gamma_{N}$ and let us choose elements $g_{0}=e$, $g_{1}, \ldots, g_{k} \in \Gamma$ such that

$$
\Gamma / \Gamma_{N}=\left\{\pi\left(g_{0}\right), \pi\left(g_{1}\right), \ldots, \pi\left(g_{k}\right)\right\} .
$$

Every element $g \in \Gamma$ can be written uniquely as $g=y g_{j}$, with $y \in \Gamma_{N}$, $0 \leq j \leq k$. We set

$$
\bar{g}=g_{j} \quad \text { and } \quad g_{N}=y .
$$

$\Gamma_{N}$ has a torsion-free subgroup $\Gamma_{N}^{1} \triangleleft \Gamma_{N}$ of finite index, that is, such that $\left|\Gamma_{N} / \Gamma_{N}^{1}\right|<\infty$ (cf. [35]). Let $\Gamma_{N}^{2}=\bigcap_{0 \leq i \leq k} g_{i} \Gamma_{N}^{1} g_{i}^{-1}$. Then $\Gamma_{N}^{2}$ is still nilpotent and torsion free. Furthermore, $\Gamma_{N}^{2} \triangleleft \Gamma$ and $\left|\Gamma / \Gamma_{N}^{2}\right|<\infty$. So by replacing $\Gamma_{N}$ with $\Gamma_{N}^{2}$, if necessary, we assume that $\Gamma_{N}$ has the following properties:

1. $\Gamma_{N} \triangleleft \Gamma$;

2. $\left|\Gamma / \Gamma_{N}\right|<\infty$;

3. $\Gamma_{N}$ is finitely generated, nilpotent and torsion free.

Let $U_{\Gamma_{N}} \subseteq \Gamma_{N}$ be a finite and symmetric subset which generates $\Gamma_{N}$ and set

$$
|x|_{\Gamma_{N}}=\min \left\{n \in \mathbb{N}: x \in U_{\Gamma_{N}}^{n}\right\}, \quad x \in \Gamma_{N} .
$$

Then there is a $c \geq 1$ such that

$$
\frac{1}{c}|x|_{\Gamma_{N}} \leq\left|x g_{i}\right|_{\Gamma} \leq c|x|_{\Gamma_{N}}
$$

for all $x \in \Gamma_{N}$ and $0 \leq i \leq k$, or more generally

$$
\frac{1}{c}\left|x^{-1} y\right|_{\Gamma_{N}} \leq\left|g^{-1} h\right|_{\Gamma} \leq c\left|x^{-1} y\right|_{\Gamma_{N}}
$$

for all $g=x g_{i}, h=y g_{j} \in \Gamma, x, y \in \Gamma_{N}, 0 \leq i, j \leq k$.

Property 3 above implies that $\Gamma_{N}$ is isomorphic to (and hence can be identified with) a uniform lattice in a simply connected nilpotent Lie group $N$ (cf. [35]). Note that $N / \Gamma_{N}$ is a compact neighborhood. Let us fix a fundamental domain $\Omega$ for $\Gamma_{N}$ and let $d g$ be the Haar measure on $N$ which satisfies dg-measure $(\Omega)=\operatorname{vol}\left(N / \Gamma_{N}\right)=1$.

Let $V$ be a compact neighborhood of the identity element $e$ of $N$ and set

$$
|x|_{N}=\min \left\{n \in \mathbb{N}: x \in V^{n}, x \in N\right\} .
$$

Then there is a $c \geq 1$ such that

$$
\frac{1}{c}|x|_{\Gamma_{N}} \leq|x|_{N} \leq c|x|_{\Gamma_{N}}, \quad x \in \Gamma_{N} .
$$

The isomorphisms $y \rightarrow g_{i} y g_{i}^{-1}, 0 \leq k \leq k$, can be extended to isomorphisms of $N$ (cf. [35]). So we can consider the group

$$
G=\left\{y g_{i}, y \in N, 0 \leq i \leq k\right\}
$$


with multiplication law defined by

$$
x g_{i} y g_{j}=x g_{i} y g_{i}^{-1}\left(g_{i} g_{j}\right)_{N} \overline{g_{i} g_{j}}, \quad x, y \in N, 0 \leq i, j \leq k .
$$

If $\Gamma$ is nilpotent, then a better way to proceed is to consider the torsion subgroup $\tau(\Gamma)$ of $\Gamma$ (cf. [12]). $\tau(\Gamma)$ is the set of elements of finite order in $\Gamma$, it is a normal subgroup of $\Gamma$ and $\Gamma / \tau(\Gamma)$ is torsion free. So, we can set $\Gamma_{N}=\Gamma / \tau(\Gamma)$.

Let $\mathfrak{n}$ be the Lie algebra of $N$. We identify $\mathfrak{n}$ with the left invariant vector fields on $N$.

By a left invariant sub-Laplacian on $N$, we mean an operator

$$
L=-\left(E_{1}^{2}+\cdots+E_{p}^{2}\right)+E_{0},
$$

where $E_{0}, E_{1}, \ldots, E_{p}$ are left invariant vector fields on $N$ and where the vector fields $E_{1}, \ldots, E_{p}$ satisfy Hörmander's condition; that is, they generate together with their successive Lie brackets $\left.\left[E_{i_{1}},\left[E_{i_{2}},\left[\ldots, E_{i_{k}}\right] \ldots\right]\right]\right], 1 \leq i_{j} \leq p, 1 \leq$ $j \leq k$, the Lie algebra $\mathfrak{n}$ of $N$.

We shall say that $L$ is centered if $E_{0} \in[\mathfrak{n}, \mathfrak{n}]$.

Let us fix a discrete probability measure $\mu$ on $\Gamma$, let $\operatorname{supp}(\mu)=\{g \in G$ : $\mu(g)>0\}$ and let us assume that the following hold:

1. $|\operatorname{supp}(\mu)|<\infty$;

2. $U \subseteq \operatorname{supp}(\mu)$;

3. $\mu$ is centered.

Our goal is to associate with $\mu$ a centered left invariant sub-Laplacian $L_{H \mu}$ on $N$, in such a way that the asymptotic behavior of the convolution powers $\mu^{* n}$ can be compared to the large-time behavior of the heat kernel $p_{t}^{H \mu}(x, y)$ of $L_{H \mu}$. If $\Gamma=\Gamma_{N}$ or $G$ is nilpotent, then the definition of $L_{H \mu}$ is rather straightforward (cf. [19]). In this case we use the notation $L_{\mu}$ and $p_{t}^{\mu}(x, y)$ instead of $L_{H \mu}$ and $p_{t}^{H \mu}(x, y)$, respectively. If $\Gamma$ is not nilpotent, then $L_{H \mu}$ is defined by a method inspired by the theory of the homogenization (cf. $[13,26]$ ). We call $L_{H \mu}$ the homogenized sub-Laplacian (associated with $\mu$ ).

1.4. Notation. Given another measure $v$ we define the convolution $\mu * v$ by $\mu * v(x)=\sum_{y \in \Gamma} \mu(y) v\left(y^{-1} x\right) d y, x \in \Gamma$.

Given a kernel $K(x, y)$ we set

$$
K(x, A)=\sum_{y \in A} K(x, y) \quad \text { and } \quad K f(x)=\sum_{y \in \Gamma} K(x, y) f(y) .
$$

If $S(x, y)$ is another kernel, then we denote by $K S$ the kernel

$$
K S(x, y)=\sum_{z \in \Gamma} K(x, z) S(z, y)
$$


We also set

$$
\begin{aligned}
\|K\|_{1} & =\sup \left\{\|K(x, .)\|_{1},\|K(., y)\|_{1} ; x, y \in \Gamma\right\}, \\
\|K\|_{\infty} & =\sup \{|K(x, y)|: x, y \in \Gamma\} .
\end{aligned}
$$

To simplify the notation, we set $\mu^{* n}=\mu^{n}, n \in \mathbb{N}$, and $\mu^{0}=\delta_{e}$, where $\delta_{x}$ is the Dirac mass at $x$. We also denote by $\mu^{n}$ the kernel

$$
\mu^{n}(x, y)=\mu^{* n}\left(x^{-1} y\right), \quad x, y \in \Gamma .
$$

For $n=1$, we just write $\mu(x, y)$ instead of $\mu^{1}(x, y)$.

Note that $\mu^{n+1}=\mu \mu^{n}$ and that

$$
\mu^{n} f(x)=\sum_{y \in \Gamma} \mu^{n}(x, y) f(y)=\sum_{y \in \Gamma} \mu^{* n}(y) f(x y) .
$$

We say that a function $u$ is $\mu$-harmonic in $A \subseteq \Gamma$ if $\mu u(x)=u(x), x \in A$.

We say that a function $u$ is a space-time $\mu$-harmonic function in $A \subseteq \mathbb{Z} \times \Gamma$ if $(\mu u(n, \cdot))(x)=u(n+1, x),(n, x) \in A$.

We denote by $\partial_{1}$ and $\partial_{z}, z \in \Gamma$, respectively the difference operators

$$
\partial_{1} u(n, x)=u(n+1, x)-u(n, x) \quad \text { and } \quad \partial_{z} u(n, x)=u(n, x z)-u(n, x) .
$$

Note that $u$ is a space-time $\mu$-harmonic function if and only if $\left(\partial_{1}+(I-\mu)\right) u=0$.

We also set

$$
\partial_{z} \mu^{* n}(x)=\mu^{* n}(x z)-\mu^{* n}(x) \quad \text { and } \quad \partial_{1} \mu^{* n}(x)=\mu^{*(n+1)}(x)-\mu^{* n}(x) .
$$

If $A \subseteq \Gamma$, then we set

$$
\nabla_{A} u(n, x)=\sup \left\{\left|\partial_{z} u(n, x)\right| ; z \in U\right\} .
$$

We say that a function $f$ is of type $\mathrm{P}$ if $f(x g)=f(g), x \in \Gamma_{N}, g \in \Gamma$.

If $f$ is such a function, then we denote by $\langle f\rangle$ its mean value

$$
\langle f\rangle=\frac{1}{k+1} \sum_{0 \leq i \leq k} f\left(g_{i}\right) .
$$

Note that if $f$ is a function of type $\mathrm{P}$, then $\mu f$ is also a function of type P. If we also have $\langle f\rangle=0$, then the function

$$
u=\sum_{n \geq 0} \mu^{n} f
$$

is well defined and satisfies

$$
(I-\mu) u=f
$$

If $\Gamma$ is nilpotent and we set $\Gamma_{N}=\Gamma / \tau(\Gamma)$, then the type $\mathrm{P}$ functions will just be the constant functions. 
If $K(x, y)$ is a kernel initially defined on $N$, then we use the same notation $K(x, y)$ to denote its restriction to $\Gamma_{N}$ and its extension to $\Gamma$. The extension of $K(x, y)$ to $\Gamma$ is defined by

$$
K\left(x g_{i}, y g_{j}\right)=\frac{1}{k+1} K(x, y), \quad x, y \in N, 0 \leq i, j \leq k .
$$

If $\Gamma$ is nilpotent and we set $\Gamma_{N}=\Gamma / \tau(\Gamma)$, then we extend $K(x, y)$ to $\Gamma$ by setting

$$
K(z x, w y)=\frac{1}{|\tau(\Gamma)|} K(\dot{x}, \dot{y}),
$$

where $z, w \in \tau(\Gamma)$ and $\dot{x}=x \tau(\Gamma), \dot{y}=y \tau(\Gamma), x, y \in \Gamma$.

In particular, we use this notation for the heat kernels $p_{t}^{H \mu}(x, y), p_{t}^{\mu}(x, y)$ and their derivatives $X_{1} \cdots X_{n} p_{t}^{H \mu}(x, y), X_{1} \cdots X_{n} p_{t}^{\mu}(x, y), X_{1}, \ldots, X_{n} \in \mathfrak{n}$.

A function $f$ on $\Gamma_{N}$ will be extended to $\Gamma$ by setting

$$
f\left(x g_{i}\right)=f(x), \quad x \in \Gamma_{N}, 0 \leq i \leq k .
$$

If $\Gamma$ is nilpotent and we set $\Gamma_{N}=\Gamma / \tau(\Gamma)$, then we extend $f$ to $\Gamma$ by setting $f(z x)=f(\dot{x})$, for $z \in \tau(\Gamma)$ and $\dot{x}=x \tau(\Gamma), x \in \Gamma$.

We do this, in particular, when $f$ is a harmonic function or a polynomial.

We denote by $\llbracket a, b \rrbracket$ the interval $[a, b] \cap \mathbb{Z}$.

Given a nonempty subset $A$ of $\Gamma$ we set $A^{0}=\{e\}$ and $A^{r}=A^{[r]}, r>0$.

The different constants are always denoted by the same letter $c$. When their dependence or independence is significant, it is clearly stated.

1.5. A parabolic Harnack inequality. The following Harnack inequality plays a central role in this article.

THEOREM 1.2. For all $a, b \geq 1$ there are $\beta>\alpha>1, c>1$ and $\lambda>0$ such that, for all $r \geq 1$ and all $u \geq 0$ satisfying

$$
\left(\partial_{1}+(I-\mu)\right) u=0 \quad \text { in } \llbracket 0,\left(\beta+b^{2}\right) r^{2} \rrbracket \times U^{c r},
$$

we have

$$
\sup \left\{u ; \llbracket \alpha r^{2},\left(\alpha+a^{2}\right) r^{2} \rrbracket \times U^{a r}\right\} \leq \lambda \inf \left\{u ; \llbracket \beta r^{2},\left(\beta+b^{2}\right) r^{2} \rrbracket \times U^{b r}\right\} .
$$

For the case when $\mu$ is symmetric [i.e., $\mu\left(x^{-1}\right)=\mu(x), x \in \Gamma$ ], the above inequality was proved in [23] by a different method.

We prove this inequality by adapting the method of Krylov and Safonov [29]. This method uses certain information on the growth of the positive space-time $\mu$-harmonic functions. To obtain this information we use the following three results. 
The first two results concern the distribution of the mass of the convolution powers $\mu^{* n}$ as $n \rightarrow \infty$ :

PROPOSITION 1.3. For all $a>1$ there are $r_{0} \geq 1$ and $\partial>0$ such that

$$
\sum_{y \in U^{r}} \mu^{n}(x, y)>\partial,
$$

for all $(n, x) \in \llbracket a^{-2} r^{2}, a^{2} r^{2} \rrbracket \times U^{a r}, r \geq r_{0}$.

PROPOSITION 1.4. For all $\partial>0$ there is an $a>1$ such that

$$
\sum_{y \notin U^{a r}} \mu^{n}(e, y)<\partial,
$$

for all $k \in \llbracket 1, r^{2} \rrbracket$.

The third result is a theorem of Varopoulos [49] which asserts that the convolution powers $\mu^{* n}$ decay with a certain uniform speed as $n \rightarrow \infty$ :

THEOREM 1.5 [49]. Let $\mu$ be a (not necessarily centered) probability measure whose support generates $\Gamma$. Then there is a constant $c>0$ such that

$$
\left\|\mu^{* n}\right\|_{\infty} \leq c n^{-D / 2}, \quad n \in \mathbb{N} .
$$

If $u$ is a function defined on $B \subseteq \mathbb{Z} \times \Gamma$, then let us set

$$
\operatorname{Osc}(u, B)=\sup \{|u(k, x)-u(m, y)|:(k, x),(m, y) \in B\} .
$$

To prove Theorem 1.2, we proceed as follows. Using the above three results, we prove an analogue of the first growth lemma of [29]. From this and arguing in the same way as in [29] we obtain a second growth lemma.

A direct consequence of the second growth lemma is the following:

PROPOSITION 1.6. There are $c \geq 1$ and $\gamma \in(0,1]$ such that, for all $t \in \mathbb{R}$, $r \geq 1$ and all functions $u$ satisfying $\left(\partial_{1}+(I-\mu)\right) u=0$ in $\llbracket t-c^{2} r^{2}, t \rrbracket \times U^{c r}$,

$$
\operatorname{Osc}\left(u, \llbracket t-r^{2}, t \rrbracket \times U^{r}\right) \leq \gamma \operatorname{Osc}\left(u, \llbracket t-c^{2} r^{2}, t \rrbracket \times U^{c r}\right) .
$$

Theorem 1.2 follows from the second growth lemma and the above proposition by a standard argument (see, e.g., [7, 30, 40, 44]). It can also be proved by arguing in the same way as in [29] (but this is less obvious).

An immediate consequence of (1.9) and (1.13) is the following:

COROLlARY 1.7. Every positive $\mu$-harmonic function is constant. 
1.6. Gaussian estimates. Making use of the Harnack inequality (1.9) we prove the following upper Gaussian estimate:

THEOREM 1.8. There is a constant $c>0$ such that

$$
\mu^{* n}(x) \leq c n^{-D / 2} \exp \left(-\frac{|x|_{\Gamma}^{2}}{c n}\right), \quad x \in \Gamma, n \in \mathbb{N} .
$$

Once we have the upper Gaussian estimate then, again by using the Harnack inequality (1.9), we can obtain a lower Gaussian estimate (cf. [23] and [50], pages $47-50)$ :

COROLlaRY 1.9. There is a constant $c>0$ such that

$$
\mu^{* n}(x) \geq \frac{1}{c} n^{-D / 2} \exp \left(-c \frac{|x|_{\Gamma}^{2}}{n}\right)
$$

for all $n \in \mathbb{N}$ and $x \in \Gamma$ satisfying $|x|_{\Gamma} \leq n / c$.

Combining (1.2) and (1.14) we have the following:

COROLlaRY 1.10. Let us assume that $\mu$ is not centered and let $\beta_{\mu}$ and $\chi$ be as in Lemma 1.1. Then there is a $c>0$ such that

$$
\mu^{* n}(x) \leq c n^{-D / 2} \exp \left(-\beta_{\mu} n\right) \chi(x) \exp \left(-\frac{|x|_{\Gamma}^{2}}{c n}\right)
$$

for all $x \in \Gamma$ and $n \in \mathbb{N}$.

1.7. A Taylor formula for the space-time $\mu$-harmonic functions. Using the exponential coordinates we shall identify $N$, as a differential manifold, with $\mathbb{R}^{q}$. So a monomial $P(x)$ on $N$ will be just a monomial on $\mathbb{R}^{q}$. A monomial $P(x)$ on $\Gamma_{N}$ will be just the restriction to $\Gamma_{N}$ of a monomial $P(x)$ on $N$. We extend the monomials $P(x)$ to $\Gamma$ by setting $P\left(x g_{i}\right)=P(x), x \in \Gamma_{N}, 1 \leq i \leq k$.

In the rest of this article, we do not make any distinction between the restriction of a monomial $P(x)$ to $\Gamma_{N}$ and its extension to $\Gamma$.

For every monomial $P(x)$, there are an integer $d \geq 0$ and a constant $c>0$ such that

$$
\frac{1}{c} n^{d} \leq \sup \left\{|P(x)|, x \in U^{n}\right\} \leq c n^{d}, \quad n \in \mathbb{N} .
$$

We say then that $P(x)$ has homogeneous degree $\operatorname{deg}_{H} P=d$.

We say that $P(t, x)$ is a monomial on $\mathbb{Z} \times \Gamma$ (resp. $\mathbb{R} \times G$ ) if $P(t, x)=t^{m} Q(x)$, with $Q(x)$ a monomial on $\Gamma$ (resp. $G$ ). We define the homogeneous degree $\operatorname{deg}_{H} P(t, x)$ of $P(t, x)$ by

$$
\operatorname{deg}_{H} P(t, x)=2 m+\operatorname{deg}_{H} Q(x) .
$$


By polynomials we of course mean linear combinations of monomials. The homogeneous degree of a polynomial is therefore the maximum of the homogeneous degrees of its monomials.

To fix the notation, we use

$$
P_{0}(t, x), P_{2}(t, x), \ldots, P_{v_{d}}(t, x)
$$

from now on to denote the monomials with homogeneous degree less than or equal to $d$. With every such monomial $P_{i}(t, x)$ we associate another, more convenient "corrected" monomial $Q_{P_{i}}^{\psi}(t, x)$ written as

$$
Q_{P_{i}}^{\psi}(t, x)=P_{i}(t, x)+\sum_{0 \leq j \leq v_{k-1}} \psi_{j}^{i}(x) P_{j}(t, x),
$$

where $k=\operatorname{deg}_{H} P_{i}$ and where the functions $\psi_{j}^{i}$ are of type P.

Note that when $\Gamma$ is nilpotent the $\psi_{j}^{i}$ will just be constant functions.

The following result gives a Taylor formula for the space-time $\mu$-harmonic functions.

THEOREM 1.11. For all $n \in \mathbb{N}$ there is a constant $c_{n}>0$ such that, for all $R \geq r \geq 1$ and all functions $u$ satisfying

$$
\left(\partial_{1}+(I-\mu)\right) u=0 \quad \text { in } \llbracket-R^{2}, R^{2} \rrbracket \times U^{R},
$$

we have

$$
\begin{aligned}
& \sup \left\{\left|u-\sum_{0 \leq i \leq \nu_{n}} A_{i} R^{-\operatorname{deg}_{H} P_{i}} Q_{P_{i}}^{\psi}\right| ; \llbracket\left[r^{2}, r^{2} \rrbracket \times U^{r}\right\}\right. \\
& \leq c_{n}\left(\frac{R}{r}\right)^{-(n+1)}\|u\|_{\infty},
\end{aligned}
$$

where the constants $A_{i}$ satisfy

$$
\left|A_{i}\right| \leq c_{n}\|u\|_{\infty}
$$

for all $0 \leq i \leq v_{n}$, and

$$
\left(\partial_{1}+(I-\mu)\right)\left(\sum_{v_{d-1}<i \leq v_{d}} A_{i} Q_{P_{i}}^{\psi}\right)=0,
$$

for all $1 \leq d \leq n$

The proof of the above result is based on ideas of Avellaneda and Lin (cf. [9, 10]). These ideas have already been used in the context of Lie groups in $[3,4,8]$. The interest of the method lies in the fact that we do not make use of any a priori control on the differences. 
1.8. $\mu$-harmonic functions of polynomial growth. We say that a function $u$ on $\Gamma$ grows polynomially if there is a $c>0$ such that

$$
\sup \left\{|u| ; U^{n}\right\} \leq c n^{c}, \quad n \in \mathbb{N} .
$$

The following result is a consequence of Theorem 1.11:

THEOREM 1.12. Every $\mu$-harmonic function $u$ which grows polynomially is equal to a linear combination of the monomials $Q_{P_{i}}^{\psi}$.

A result of this type was first proved by Avellaneda and Lin [10] in the case of differential operators with periodic coefficients in $\mathbb{R}^{n}$. It was generalized in [8] in the context of connected Lie groups of polynomial volume growth, where it was used to prove a Sobolev inequality. We state below the discrete analogue of that inequality. The proof is similar and is omitted.

COROLlaRY 1.13. Let $f$ be a function, not necessarily with compact support, such that $\nabla_{U} f \in L^{p}, 1<p<D$. Then there are a universal constant $c>0$ and a constant $C_{f}$ depending on $f$ such that $f-C_{f} \in L^{D p /(D-p)}$, and

$$
\left\|f-C_{f}\right\|_{D p /(D-p)} \leq c\left\|\nabla_{U} f\right\|_{p} .
$$

1.9. Harnack inequalities for the differences. A consequence of Theorem 1.11 is the following result:

THEOREM 1.14. For all $a, b \geq 1$ and all $k \in \mathbb{N}$ there are $\beta>\alpha>1, c>1$ and $\lambda>0$ such that, for all $z \in U, r \geq 1$ and all $u \geq 0$ satisfying

$$
\left(\partial_{1}+(I-\mu)\right) u=0 \quad \text { in } \llbracket 0, c r^{2} \rrbracket \times U^{c r},
$$

we have

$$
\begin{aligned}
& \sup \left\{\left|\partial_{1}^{k} \partial_{z} u\right| ; \llbracket \alpha r^{2},\left(\alpha+a^{2}\right) r^{2} \rrbracket \times U^{a r}\right\} \\
& \quad \leq \lambda r^{-2 k-1} \inf \left\{u ; \llbracket \beta r^{2},\left(\beta+b^{2}\right) r^{2} \rrbracket \times U^{b r}\right\}
\end{aligned}
$$

If $\Gamma$ is nilpotent, then we can also control higher order spacial differences:

THEOREM 1.15. If $\Gamma=\Gamma_{N}$, then for all $a, b \geq 1$ and all $k, m \in \mathbb{N}$ there are $\beta>\alpha>1, c>1$ and $\lambda>0$ such that, for all $z_{1}, \ldots, z_{m} \in U, r \geq 1$ and all $u \geq 0$ satisfying

$$
\left(\partial_{1}+(I-\mu)\right) u=0 \quad \text { in } \llbracket 0, c r^{2} \rrbracket \times U^{c r},
$$

we have

$$
\begin{aligned}
& \sup \left\{\left|\partial_{1}^{k} \partial_{z_{1}} \cdots \partial_{z_{m}} u\right| ; \llbracket \alpha r^{2},\left(\alpha+a^{2}\right) r^{2} \rrbracket \times U^{a r}\right\} \\
& \quad \leq \lambda r^{-2 k-m} \inf \left\{u ; \llbracket \beta r^{2},\left(\beta+b^{2}\right) r^{2} \rrbracket \times U^{b r}\right\} .
\end{aligned}
$$


Note that if $\Gamma$ is not nilpotent, then (1.21) is not necessarily true for $m \geq 2$. This is due to the existence of the functions $\psi_{j}^{i}$ in the definition of the monomials $Q_{P_{i}}^{\psi}$ [see Sections 1.7 and 19 as well as (1.30) below].

1.10. Berry-Esseen estimates. Let $p_{t}^{H \mu}(x, y)$ be the heat kernel of the homogenized sub-Laplacian $L_{H \mu}$ associated with $\mu$. As mentioned in Section 1.4, we extend $p_{t}^{H \mu}(x, y)$ to $G$, by setting

$$
p_{t}^{H \mu}\left(x g_{i}, y g_{j}\right)=\frac{1}{k+1} p_{t}^{H \mu}(x, y), \quad x, y \in N, 0 \leq i, j \leq k .
$$

We have the following analogue of the Berry-Esseen theorem (cf. [20, 26, 28, 34, 51]):

THEOREM 1.16. There is a $c>0$ such that

$$
\left|\mu^{n}(x, y)-p_{n}^{H \mu}(x, y)\right| \leq c n^{-(D+1) / 2}
$$

for all $x, y \in \Gamma$ and $n \in \mathbb{N}$.

The reader can observe that by a straightforward adaptation of the proof of the above result we can also obtain a similar $L^{1}$ estimate, that is, that there is a $c>0$ such that

$$
\left\|\mu^{n}-p_{n}^{H \mu}\right\|_{1} \leq c / \sqrt{n}, \quad n \in \mathbb{N} .
$$

It was proved in $[2,7]$ that there are constants $c, C_{L} \geq 0$ such that

$$
\left|p_{t}^{H \mu}(e, e)-C_{L_{H \mu}} t^{-D / 2}\right| \leq c t^{-(D+1) / 2}, \quad t \geq 1 .
$$

Combining (1.23) and (1.24) we have the following:

COROLlaRY 1.17. There are constants $c, C_{\mu} \geq 0$ such that

$$
\left|\mu^{* n}(e)-C_{\mu} n^{-D / 2}\right| \leq c n^{-(D+1) / 2}, \quad n \in \mathbb{N} .
$$

Combining (1.2) and (1.25) we have the following:

COROLLARY 1.18. Let us assume that $\mu$ is not centered and let $\beta_{\mu}$ be as in Lemma 1.1. Then there are constants $c, C_{\mu} \geq 0$ such that

$$
\left|\mu^{* n}(e)-C_{\mu} n^{-D / 2} e^{-\beta_{\mu} n}\right| \leq c n^{-(D+1) / 2} e^{-\beta_{\mu} n}, \quad n \in \mathbb{N} .
$$

By interpolating (1.14) and (1.22) we can have the following: 
COROLlaRY 1.19. For all $\varepsilon \in(0,1)$ there is a $c>0$ such that

$$
\left|\mu^{n}(x, y)-p_{n}^{H \mu}(x, y)\right| \leq c n^{-(D+\varepsilon) / 2} \exp \left(-\frac{\left|x^{-1} y\right|_{\Gamma}^{2}}{c n}\right)
$$

for all $x, y \in \Gamma$ and $n \in \mathbb{N}$.

Concerning the space and time differences we have the following results:

THEOREM 1.20. There is a constant $c>0$ such that

$$
\left|\partial_{1} \mu^{n}(x, y)-\partial_{1} p_{n}^{H \mu}(x, y)\right| \leq c n^{-(D+3) / 2}
$$

for all $x, y \in \Gamma$ and $n \in \mathbb{N}$.

THEOREM 1.21. If $\Gamma$ is nilpotent, then there is a constant $c>0$ such that, for all $z \in U$ and all $x, y \in \Gamma$ and $n \in \mathbb{N}$,

$$
\left|\partial_{z} \mu^{n}(x, y)-\partial_{z} p_{n}^{\mu}(x, y)\right| \leq c n^{-(D+2) / 2} .
$$

If $\Gamma$ is not nilpotent, then the situation is quite different. More precisely, if $X_{1}, \ldots, X_{q}$ is a convenient basis of $\mathfrak{n}$ and if $\psi^{1}, \ldots, \psi^{n_{1}}$ are the associated first order correctors (see Section 14 for the exact definitions), then we have the following result:

THEOREM 1.22. There is $a c>0$ such that, for all $z \in U$ and all $x, y \in \Gamma$ and $n \in \mathbb{N}$,

$$
\begin{aligned}
& \left|\partial_{z} \mu^{n}(x, y)-\partial_{z} p_{n}^{H \mu}(x, y)-\sum_{1 \leq j \leq n_{1}}\left(\partial_{z} \psi^{j}(x)\right) X_{j} p_{n}^{H \mu}(x, y)\right| \\
& \leq c n^{-(D+2) / 2} \text {. }
\end{aligned}
$$

Combining (1.14) and (1.30) we have the following:

COROLlary 1.23. For all $\varepsilon \in(0,1)$ there is a constant $c>0$ such that, for all $z \in U$ and all $x, y \in \Gamma$ and $n \in \mathbb{N}$,

$$
\begin{aligned}
& \left|\partial_{z} \mu^{n}(x, y)-\partial_{z} p_{n}^{H \mu}(x, y)-\sum_{1 \leq j \leq n_{1}}\left(\partial_{z} \psi^{j}(x)\right) X_{j} p_{n}^{H \mu}(x, y)\right| \\
& \quad \leq c n^{-(D+1+\varepsilon) / 2} \exp \left(-\frac{\left|x^{-1} y\right|_{\Gamma}^{2}}{c n}\right) .
\end{aligned}
$$

Inequalities (1.27) and (1.31) above actually hold with $\varepsilon=1$. This can be proved by arguing in a similar way as in the proofs of Theorems 1.16 and 1.22. However, the proofs become much more technical, while (1.27) and (1.31) are sufficient for the application that we have in mind, namely the proof of Theorem 1.24. 
1.11. Riesz transforms. Let us denote by $(I-\mu)^{-1 / 2}$ the operator defined by $(I-\mu)^{-1 / 2}=\sum_{n \geq 0} a_{n} \mu^{n}$, where the $a_{n}$ 's are as in $(1-t)^{-1 / 2}=\sum_{n \geq 0} a_{n} t^{n}$.

THEOREM 1.24. For all $z \in \Gamma$ the Riesz transform operators $R_{z}=\partial_{z}(I-$ $\mu)^{-1 / 2}$ and $R_{z}^{*}=(I-\mu)^{-1 / 2} \partial_{z}$ are bounded on $L^{p}$, for $1<p<\infty$ and from $L^{1}$ to weak- $L^{1}$.

If $\Gamma$ is nilpotent, then we can also consider higher order Riesz transforms.

THEOREM 1.25. If $\Gamma$ is nilpotent, then for all $z_{1}, \ldots, z_{k} \in \Gamma$ the Riesz transform operators $R_{k}=\partial_{z_{1}} \cdots \partial_{z_{k}}(I-\mu)^{-k / 2}$ and $R_{k}^{*}=(I-\mu)^{-k / 2} \partial_{z_{k}} \cdots \partial_{z_{1}}$, are bounded on $L^{p}$, for $1<p<\infty$ and from $L^{1}$ to weak- $L^{1}$.

If $\Gamma$ is not nilpotent then, as we can see from (1.31), the second order Riesz transforms $R_{2}=\partial_{z_{1}} \partial_{z_{2}}(I-\mu)^{-1}$ and $R_{2}^{*}=(I-\mu)^{-1} \partial_{z_{2}} \partial_{z_{1}}$ may be unbounded even on $L^{2}$ (cf. [3]).

2. Organization of the article. We have tried to give the proof of the results in the simplest possible context. The proof of the parabolic Harnack inequality (1.9) from Varopoulos's theorem (Theorem 1.5) and by assuming Propositions 1.3 and 1.4, does not use any particular result from the structure of $\Gamma$ and so it is given already in Section 3. The construction of the operator $L_{H \mu}$ is much simpler when $\Gamma=\Gamma_{N}$. So those proofs that are essentially the same, whether $\Gamma=\Gamma_{N}$ or not, are only given in the case $\Gamma=\Gamma_{N}$. This is the case for the Gaussian estimate (1.14), the Taylor formula (1.18) and the main part of the proof of Propositions 1.3 and 1.4 .

The proof of the Berry-Esseen estimate (1.23) is much more complicated when $\Gamma \neq \Gamma_{N}$. So, to illustrate the ideas better, we also give the proof in the case $\Gamma=\Gamma_{N}$.

3. The proof of the Harnack inequality from Varopoulos's theorem and Propositions 1.3 and 1.4. In this section we give the proof of Theorem 1.2 from Varopoulos's theorem (Theorem 1.5) and by assuming Propositions 1.3 and 1.4. This has already been done in [7] in the context of left invariant sub-Laplacians on connected Lie groups of polynomial volume growth. We give below an adaptation of that proof in the context of discrete groups.

We first prove an analogue of the first growth lemma of [29] by using (1.10), (1.11) and (1.12).

Next, we prove an analogue of the second growth lemma of [29]. To do this, we follow closely [29] and we adapt in our context their covering lemmas.

The proof of Proposition 1.6 and of Theorem 1.2 from the second growth lemma is standard in the literature (cf. [7, 30, 40, 44]; since it is also long, it will be omitted. We point out again that the argument given in [29] can also be used. 
If $A \subseteq \mathbb{Z} \times \Gamma$, then we denote by $|A|$ the number of its elements.

If $A \subseteq \mathbb{R} \times \Gamma$, then we set

$$
|A|=\sum_{x \in \Gamma}\left|A_{x}\right|,
$$

where $\left|A_{x}\right|$ is the Lebesgue measure of $A_{x}=A \cap \mathbb{R} \times\{x\}$.

3.1. The first growth lemma. If $B \subseteq \mathbb{Z} \times \Gamma, A \subseteq B$ and $(t, x) \in B$ then, adopting the notation of [29], we set

$$
\begin{array}{r}
\Psi((t, x), A, B)=\inf \{u(t, x): u \geq 0, u(s, y) \geq 1 \text { for }(s, y) \in A \\
\text { and } \left.\left(\partial_{1}+(I-\mu)\right) u=0 \text { in } B\right\} .
\end{array}
$$

If $A^{\prime} \subseteq B$, then we set

$$
\Psi\left(A^{\prime}, A, B\right)=\inf \left\{\Psi((t, x), A, B):(t, x) \in A^{\prime}\right\} .
$$

Note that if $v \geq 0, u=\mu v$ and $a=\min \{\mu(x), x \in U\}$, then

$$
u(x) \geq \operatorname{av}(x y), \quad y \in U .
$$

LEMMA 3.1 (First growth lemma). For all $a>1$, there are $r_{0} \geq 1, c>a$ and $\partial, \xi \in(0,1)$ such that

$$
\Psi\left(\llbracket a^{-2} r^{2}, a^{2} r^{2} \rrbracket \times U^{a r}, A, \llbracket 0, a^{2} r^{2} \rrbracket \times U^{c r}\right)>\delta
$$

for all $r \geq r_{0}$ and every $A \subseteq \llbracket 0, r^{2} \rrbracket \times U^{r}$ satisfying

$$
|A|>\xi\left|\llbracket 0, r^{2} \rrbracket \times U^{r}\right| .
$$

An immediate consequence of (3.1) and (3.2) is the following:

COROLlaRY 3.2. For all $a>1$ there are $c>a, r_{0} \geq 1, m \in \mathbb{N}$ and $\partial>0$ such that for all $r \geq r_{0}$ and all $u \geq 0$ satisfying

$$
\left(\partial_{1}+(I-\Phi)\right) u=0 \quad \text { in } \llbracket 0, a^{2} r^{2} \rrbracket \times U^{c r}
$$

we have

$$
\inf \left\{u ; \llbracket a^{-2} r^{2}, a^{2} r^{2} \rrbracket \times U^{a r}\right\} \geq \delta u(1, e) r^{-m} .
$$

Moreover, if for some $1 \leq R \leq r$,

$$
\inf \left\{u ; \llbracket 0, R^{2} \rrbracket \times U^{R}\right\} \geq 1,
$$

then

$$
\inf \left\{u ; \llbracket a^{-2} r^{2}, a^{2} r^{2} \rrbracket \times U^{a r}\right\} \geq \delta\left(\frac{R}{r}\right)^{m}
$$


3.2. Proof of Lemma 3.1. The following lemma is an immediate consequence of Theorem 1.5 and Proposition 1.3.

LEMMA 3.3. For all $a>1$ there are $r_{0} \geq 1, \partial>0$ and $\xi \in(0,1)$ such that

$$
\sum_{y \in A} \mu^{n}(x, y)>\delta
$$

for all $r \geq r_{0},(n, x) \in \llbracket a^{-2} r^{2}, a^{2} r^{2} \rrbracket \times U^{a r}$ and $A \subseteq U^{r}$ satisfying

$$
|A|>\xi\left|U^{r}\right| \text {. }
$$

Let $Z_{n}$ be the random walk with transition probabilities

$$
P\left[Z_{n+1}=y \mid Z_{n}=x\right]=\mu(x, y) .
$$

Let us also denote by $P_{x}, x \in G$, the probability measures satisfying

$$
P_{x}\left[Z_{0}=x\right]=1 \quad \text { and } \quad P_{x}\left[Z_{n}=y\right]=\mu^{n}(x, y) .
$$

If $r>0$ and $x \in \Gamma$, then we denote by $\tau_{r}^{x}$ the first exit time

$$
\tau_{r}^{x}=\min \left\{n \in \mathbb{N}: Z_{n} \notin x U^{r}\right\} .
$$

LEMMA 3.4. For all $\varepsilon>0$ there is a constant $c=c(\varepsilon)>0$ such that, for all $r \geq 1$,

$$
P_{x}\left[\tau_{c r}^{x} \leq r^{2}\right] \leq \varepsilon
$$

ProOF. By Proposition 1.3, there is a $\delta>0$ such that, for all $r \geq 1$,

$$
\sum_{y \in U^{r}} \mu^{n}(e, y) \geq \partial, \quad 1 \leq n \leq r^{2} .
$$

Let us fix $\varepsilon>0$. Then, by Proposition 1.4, there is a $c \geq 1$ such that, for all $r \geq 1$,

$$
\sum_{y \notin U^{c r}} \mu^{r^{2}}(e, y) \leq \varepsilon \delta .
$$

By choosing a larger constant $c$ if necessary we can also assume that $x U^{r} \cap U^{c r}$ $=\varnothing$ for $x \notin U^{2 c r}, r \geq 1$.

We have

$$
\begin{aligned}
\varepsilon \delta & \geq \sum_{y \notin x U^{c r}} \mu^{r^{2}}(x, y) \\
& =P_{x}\left[Z_{r^{2}} \notin x U^{c r}\right] \\
& \geq E^{P_{x}}\left[\mu^{r^{2}-\tau_{2 c r}^{x}}\left(Z_{\tau_{2 c r}^{x}}, G \backslash U^{c r}\right) ; \tau_{2 c r}^{x} \leq r^{2}\right]
\end{aligned}
$$




$$
\begin{aligned}
& \geq E^{P_{x}}\left[\mu^{r^{2}-\tau_{2 c r}^{x}}\left(Z_{\tau_{2 c r}^{x}}, Z_{\tau_{2 c r}^{x}} U^{r}\right) ; \tau_{2 c r}^{x} \leq r^{2}\right] \\
& =E^{P_{x}}\left[\mu^{r^{2}-\tau_{2 c r}^{x}}\left(e, U^{r}\right) ; \tau_{2 c r}^{x} \leq r^{2}\right] \\
& \geq \delta P_{x}\left[\tau_{2 c r}^{x} \leq r^{2}\right]
\end{aligned}
$$

and hence

$$
P_{x}\left[\tau_{2 c r}^{x} \leq r^{2}\right] \leq \varepsilon,
$$

which proves the lemma.

Proof of Lemma 3.1. Let $t_{0} \in \llbracket 0, \frac{1}{2} a^{-2} r^{2} \rrbracket$ and let

$$
A_{t_{0}}=A \cap\left\{t_{0}\right\} \times U^{r} .
$$

Let $c>a$ and let $u \geq 0$ satisfy $u(s, y) \geq 1$, for $(s, y) \in A$ and $\left(\partial_{1}+(I-\mu)\right)$ $u=0$ in $\llbracket 0, a^{2} r^{2} \rrbracket \times U^{2 c r}$. Then, for all $(t, x) \in \llbracket a^{-2} r^{2}, a^{2} r^{2} \rrbracket \times U^{a r}$,

$$
\begin{aligned}
u(t, x) & \geq E^{P_{x}}\left[u\left(t_{0}, Z_{t-t_{0}}\right) ; \tau_{c r}^{x}>t-t_{0}\right] \\
& \geq E^{P_{x}}\left[\mathbb{1}_{A_{t_{0}}}\left(Z_{t-t_{0}}\right) ; \tau_{c r}^{x}>t-t_{0}\right] \\
& =\mu^{t-t_{0}}\left(x, A_{t_{0}}\right)-P_{x}\left[\tau_{c r}^{x} \leq t-t_{0}\right] .
\end{aligned}
$$

Now, by Lemma 3.3, there are $\partial>0, r_{0} \geq 1$ and $\xi_{0} \in(0,1)$ such that

$$
\mu^{t-t_{0}}\left(x, A_{t_{0}}\right)>2 \delta
$$

for all $(t, x) \in \llbracket a^{-2} r^{2}, a^{2} r^{2} \rrbracket \times U^{a r}$, if $r \geq r_{0}$ and $A_{t_{0}}$ satisfies

$$
\left|A_{t_{0}}\right|>\xi_{0}\left|U^{r}\right|
$$

If we assume that $|A|>\xi\left|\llbracket 0, r^{2} \rrbracket \times U^{r}\right|$, with $\xi \in\left[\xi_{0}, 1\right)$ close enough to 1 , then $A$ will always have a section $A_{t_{0}}$ with $t_{0} \in \llbracket 0, \frac{1}{2} a^{-2} r^{2} \rrbracket$ and satisfying (3.8).

Also, by Lemma 3.4, if the constant $c$ is large enough, then there is a $\delta>0$ such that

$$
P_{x}\left[\tau_{c r}^{x} \leq t-t_{0}\right]<\delta, \quad t \in \llbracket a^{-2} r^{2}, a^{2} r^{2} \rrbracket,
$$

and hence, for all $(t, x) \in \llbracket a^{-2} r^{2}, a^{2} r^{2} \rrbracket \times U^{a r}$,

$$
\begin{aligned}
u(t, x) & \geq \mu^{t-t_{0}}\left(x, A_{t_{0}}\right)-P_{x}\left[\tau_{c r}^{x} \leq t-t_{0}\right] \\
& \geq 2 \delta-\delta=\delta,
\end{aligned}
$$

which proves the lemma. 
3.3. The second growth lemma.

LEMma 3.5 (Second growth lemma). For all $b>1$, there are $\beta>1, c>1$, $\delta>0$ and $m \in \mathbb{N}$ such that

$$
\begin{aligned}
& \Psi\left(\llbracket \beta r^{2},\left(\beta+b^{2}\right) r^{2} \rrbracket \times U^{b r}, A, \llbracket 0,\left(\beta+b^{2}\right) r^{2} \rrbracket \times U^{c r}\right) \\
& \quad>\delta\left(\frac{|A|}{\left|\llbracket 1, r^{2} \rrbracket \times U^{r}\right|}\right)^{m}
\end{aligned}
$$

for all $r \geq 1$ and every $A \subseteq \llbracket 1, r^{2} \rrbracket \times U^{r}$.

The above lemma will actually be a consequence of the following:

LEMMA 3.6. For all $b \geq 1$, there are $c, \beta>0, \delta>0, \delta^{\prime}>0, \theta>0$ and $m \in \mathbb{N}$ such that for all $r \geq 1$ and every $A \subseteq \llbracket 1, r^{2} \rrbracket \times U^{r}$ either

$$
\begin{aligned}
& \Psi\left(\llbracket \beta r^{2},\left(\beta+b^{2}\right) r^{2} \rrbracket \times U^{b r}, A, \llbracket 0,\left(\beta+b^{2}\right) r^{2} \rrbracket \times U^{c r}\right) \\
& \quad>\partial\left(\frac{|A|}{\left|\llbracket 1, r^{2} \rrbracket \times U^{r}\right|}\right)^{m}
\end{aligned}
$$

or there is an $A_{0} \subseteq \llbracket 1, r^{2} \rrbracket \times U^{r}$ such that

$$
\left|A_{0}\right|>(1+\theta)|A|
$$

and

$$
\Psi\left(A_{0}, A, \llbracket 0, r^{2} \rrbracket \times U^{c r}\right)>\delta^{\prime} .
$$

3.4. Proof of Lemma 3.6. We use the notation

$$
Q(s, t, x)=\llbracket t-\frac{1}{2} s^{2}, t+\frac{1}{2} s^{2} \rrbracket \times x U^{s} .
$$

By Lemma 3.1, there are $s_{0} \geq 1, \delta_{1}, \xi \in(0,1)$ and $c_{1}>4$ such that

$$
\Psi\left(\llbracket \frac{1}{16} s^{2}, 16 s^{2} \rrbracket \times U^{4 s}, V, \llbracket 0,16 s^{2} \rrbracket \times U^{c s}\right)>\delta_{1}
$$

for all $s \geq s_{0}$ and every measurable subset $V \subseteq \llbracket 0, s^{2} \rrbracket \times U^{s}$ satisfying

$$
|V| \geq \xi\left|\llbracket 0, s^{2} \rrbracket \times U^{s}\right| .
$$

Let us fix $r_{0}>3 s_{0}$. Then by (3.1) there are $k>r_{0}^{2}, c_{2} \geq 1$ and $\delta_{2}>0$ such that

$$
\Psi\left(Q\left(r_{0}, 2 k, x\right),\{(k, x)\}, \llbracket 0,2 k+\frac{1}{2} r_{0}^{2} \rrbracket \times x U^{c_{2} r_{0}}\right)>\delta_{2} .
$$

Let us assume that $r^{2} \geq 6 k, c>c_{1}+c_{2}$ and consider the sets

$$
A_{1}=A \cap \llbracket 3 k, r^{2}-3 k \rrbracket \times U^{r} \quad \text { and } \quad A_{2}=A \backslash A_{1} .
$$

Note that for $r^{2}<6 k$ the lemma follows from (3.1).

Let $\eta \in(0,1)$ be determined later. 
Case I $\left(\left|A_{1}\right| \leq \eta|A|\right)$. Then $\left|A_{2}\right|>(1-\eta)|A|$ and hence

$$
\begin{aligned}
\frac{|A|}{\left|\llbracket 1, r^{2} \rrbracket \times U^{r}\right|} & \leq \frac{1}{1-\eta} \frac{\left|A_{2}\right|}{\left|\llbracket 1, r^{2} \rrbracket \times U^{r}\right|} \\
& \leq \frac{1}{1-\eta} \frac{\left|\llbracket 1,3 k \rrbracket \times U^{r}\right|+\left|\llbracket r^{2}-3 k, r^{2} \rrbracket \times U^{r}\right|}{\left|\llbracket 1, r^{2} \rrbracket \times U^{r}\right|} \\
& \leq \frac{6 k+2}{(1-\eta) r^{2}} .
\end{aligned}
$$

If $A \neq \varnothing$, then there is a $(t, x) \in \llbracket 1, r^{2} \rrbracket \times U^{r}$ such that $u(t, x) \geq 1$ and so (3.10) follows from (3.3).

Case II $\left(\left|A_{1}\right|>\eta|A|\right)$. Let

$$
A_{k}=\left\{(t, x):(t-k, x) \in A_{1}\right\} .
$$

Then

$$
A_{k} \subseteq \llbracket 4 k, r^{2}-2 k \rrbracket \times U^{r} \quad \text { and } \quad\left|A_{k}\right|=\left|A_{1}\right|>\eta|A| .
$$

We set

$$
A_{\delta_{2}}=\bigcup_{(t, x) \in A_{k}} Q\left(r_{0}, t, x\right) \cap \llbracket 1, r^{2} \rrbracket \times U^{r} .
$$

Then of course $A_{k} \subseteq A_{\delta_{2}}$ and, by (3.14),

$$
\Psi\left(A_{\delta_{2}}, A, \llbracket 0, r^{2} \rrbracket \times U^{c r}\right)>\delta_{2} .
$$

We consider the set of balls

$$
\begin{aligned}
\mathcal{Q}= & \left\{\llbracket t, t+s^{2} \rrbracket \times x U^{s} \subseteq \llbracket 1, r^{2} \rrbracket \times U^{r}: s \geq s_{0},\right. \\
& \left.|x|_{\Gamma}+|y|_{\Gamma} \leq r, y \in U^{s} \text { and }\left|Q \cap A_{\delta_{2}}\right| \geq \xi|Q|\right\} .
\end{aligned}
$$

With every ball $Q=\llbracket t, t+s^{2} \rrbracket \times x U^{s} \in \mathcal{Q}$ we associate a ball $Q^{0}$ as follows:

If $s+|x|_{\Gamma}<r$, then we set $Q^{\prime}=\llbracket t, t+(s+1)^{2} \rrbracket \times x U^{s+1}$. If $s+|x|_{\Gamma} \geq r$, then we consider $y_{1}, \ldots, y_{|x|_{\Gamma}} \in U$ such that $x=y_{1} \cdots y_{|x|_{\Gamma}}$ and we set $x^{\prime}=$ $y_{1} \cdots y_{|x|_{\Gamma}-1}$ and $Q^{\prime}=\llbracket t, t+(s+1)^{2} \rrbracket \times x^{\prime} U^{s+1}$. If $Q^{\prime}=\left|Q^{\prime} \cap A_{\delta_{2}}\right|<\xi\left|Q^{\prime}\right|$, then we take $Q^{0}=Q^{\prime}$. If not, then we repeat the same proceedure.

We set

$$
\mathcal{Q}^{0}=\left\{Q^{0}: Q \in \mathcal{Q}\right\} \quad \text { and } \quad W^{0}=\bigcup_{Q^{0} \in Q^{0}} Q^{0} .
$$

Note that $A_{k} \subseteq W^{0}$. 
LEMMA 3.7. There is a $\theta_{1}=\theta_{1}(\xi)>0$ such that

$$
\left|W^{0}\right|>\left(1+\theta_{1}\right)\left|A_{k}\right|
$$

and hence

$$
\left|W^{0}\right|>\left(1+\theta_{1}\right) \eta|A| .
$$

The proof of the above lemma is given later.

By using (3.13) repeatedly, we can see that there are $\delta_{3}>0, \delta_{3} \leq \min \left(\delta_{1}, \delta_{2}\right)$ and $m \in \mathbb{N}$ such that, for all $\sigma \geq 1$ and $s \geq s_{0}$,

$$
\begin{aligned}
& \Psi\left(\llbracket s^{2}, 16 \sigma^{2} s^{2} \rrbracket \times U^{4 s}, \llbracket s^{2}, 16 s^{2} \rrbracket \times U^{4 s}, \llbracket s^{2}, 16 \sigma^{2} s^{2} \rrbracket \times U^{c \sigma s}\right) \\
& \quad \geq \delta_{3} \sigma^{-m} .
\end{aligned}
$$

Let us fix $\sigma>2$ such that

$$
\left(1+\theta_{1}\right) \frac{16(\sigma-1)^{2}-1}{16(\sigma-1)^{2}}>1+\frac{\theta_{1}}{2} .
$$

If $Q^{0}=\llbracket t, t+s^{2} \rrbracket \times x U^{s} \in \mathcal{Q}^{0}$, then we set

$$
Q^{1}=\llbracket t, t+16 \sigma^{2} s^{2} \rrbracket \times x U^{s}, \quad Q^{1}=\left\{Q^{1}, Q^{0} \in Q^{0}\right\}
$$

and

$$
W^{1}=\bigcup_{Q^{1} \in \mathcal{Q}^{1}} Q^{1}
$$

We also set

$$
Q_{\mathbb{R}}^{1}=\left(t, t+16(\sigma-1)^{2} s^{2}\right) \times x U^{s}
$$

and define $\mathcal{Q}_{\mathbb{R}}^{1}$ and $W_{\mathbb{R}}^{1}$ similarly.

If $Q^{1}=\llbracket t, t+16 \sigma^{2} s^{2} \rrbracket \times x U^{s} \in \mathcal{Q}^{1}$, then we set

$$
Q^{2}=\llbracket t+s^{2}, t+16 \sigma^{2} s^{2} \rrbracket \times x U^{s}, \quad Q^{2}=\left\{Q^{2}, Q^{1} \in Q^{1}\right\}
$$

and

$$
W^{2}=\bigcup_{Q^{2} \in Q^{2}} Q^{2}
$$

We also set

$$
Q_{\mathbb{R}}^{2}=\left(t+s^{2}, t+16(\sigma-1)^{2} s^{2}\right) \times x U^{s}
$$

and define $\mathcal{Q}_{\mathbb{R}}^{2}$ and $W_{\mathbb{R}}^{2}$ similarly.

It follows from (3.15) and (3.18) that if $\delta_{4}=\delta_{1} \delta_{3} \sigma^{-m}$, then

$$
\Psi\left(W^{2}, A, \llbracket 0, c^{2} r^{2} \rrbracket \times U^{c r}\right)>\delta_{4} .
$$

Let

$$
\gamma=\frac{|A|}{\left|\llbracket 1, r^{2} \rrbracket \times U^{r}\right|}
$$

and let $\omega \in(0,1)$ be determined later. 
Case IIa $\left(\left|W^{2} \backslash \llbracket 1, r^{2} \rrbracket \times U^{r}\right| \geq \omega|A|\right)$. This assumption implies that

$$
\left|W^{2} \backslash \llbracket 1, r^{2} \rrbracket \times U^{r}\right| \geq \omega \gamma\left|\llbracket 1, r^{2} \rrbracket \times U^{r}\right| .
$$

So there is a ball

$$
Q^{2}=\llbracket t+s^{2}, t+16 \sigma^{2} s^{2} \rrbracket \times x U^{s} \in Q^{2}
$$

such that

$$
16 \sigma^{2} s^{2} \geq \omega \gamma r^{2}
$$

Now, by Corollary 3.2, for all $a_{1}>0$, there are $c \geq a_{1}, \delta_{5}>0$ and $m \in \mathbb{N}$ such that if $s_{0}$ is chosen large enough and $R \geq 4 \sigma s$,

$$
\begin{aligned}
\Psi(\llbracket t & \left.+R^{2}, t+\left(1+a_{1}^{2}\right) R^{2} \rrbracket \times x U^{a_{1} R}, Q^{2}, \llbracket t, t+\left(1+a_{1}^{2}\right) R^{2} \rrbracket \times x U^{c R}\right) \\
& \geq \delta_{5}\left(\frac{4 \sigma s}{R}\right)^{m} \\
& \geq \delta_{5} \omega^{m / 2}\left(\frac{r}{R}\right)^{m} \gamma^{m / 2} .
\end{aligned}
$$

The lemma follows from (3.21) above, by taking $a_{1}$ large enough and by replacing $R$ by an appropriate multiple of $r$.

Case IIb $\left(\left|W^{2} \backslash \llbracket 1,1+r^{2} \rrbracket \times U^{r}\right|<\omega|A|\right)$. Let us first observe that

$$
\left|W^{0}\right| \leq\left|W_{\mathbb{R}}^{1}\right|
$$

and that

$$
\left|W_{\mathbb{R}}^{2}\right| \leq\left|W^{2}\right| .
$$

The following lemma is the analogue of Lemma 2.3 in [29], page 158.

LEMMA 3.8 .

$$
\left|W_{\mathbb{R}}^{1}\right| \leq \frac{16(\sigma-1)^{2}}{16(\sigma-1)^{2}-1}\left|W_{\mathbb{R}}^{2}\right| .
$$

The proof of the above lemma is given in Section 3.6.

Combining (3.19), (3.22), (3.23) and (3.24) we have

$$
\begin{aligned}
\left|W^{2}\right| & \geq \frac{16(\sigma-1)^{2}-1}{16(\sigma-1)^{2}}\left|W^{0}\right| \\
& \geq \frac{16(\sigma-1)^{2}-1}{16(\sigma-1)^{2}}\left(1+\theta_{1}\right) \eta|A| \\
& \geq\left(1+\frac{\theta_{1}}{2}\right) \eta|A| .
\end{aligned}
$$


We set

$$
A_{0}=W^{2} \cap \llbracket 1, r^{2} \rrbracket \times U^{r}
$$

Then

$$
\begin{aligned}
\left|A_{0}\right| & =\left|W^{2} \cap \llbracket 1, r^{2} \rrbracket \times U^{r}\right| \\
& =\left|W^{2}\right|-\left|W^{2} \backslash \llbracket 1, r^{2} \rrbracket \times U^{r}\right| \\
& \geq\left|W^{2}\right|-\omega|A| \\
& \geq\left(1+\frac{\theta_{1}}{2}\right) \eta|A|-\omega|A| \\
& \geq\left[\left(1+\frac{\theta_{1}}{2}\right) \eta-\omega\right]|A| .
\end{aligned}
$$

It follows that if we chose $\eta \in(0,1)$ so that

$$
\left(1+\frac{\theta_{1}}{2}\right) \eta>1+\frac{\theta_{1}}{4}
$$

and

$$
\omega \in\left(0, \frac{\theta_{1}}{8}\right)
$$

then we would have

$$
\left|A_{0}\right|>\left(1+\frac{\theta_{1}}{8}\right)|A|
$$

which proves (3.11).

3.5. Proof of Lemma 3.7. If $Q=Q(s, t, x)=\llbracket t-\frac{1}{2} s^{2}, t+\frac{1}{2} s^{2} \rrbracket \times x U^{s}$, then we denote by $Q^{*}$ the ball

$$
Q^{*}(s, t, x)=\llbracket\left[t-\frac{25}{2} s^{2}, t+\frac{25}{2} s^{2} \rrbracket \times x U^{5 s} .\right.
$$

Using a standard Vitalli type of argument (cf., e.g., [7, 27, 39]) we can prove that there is a finite sequence of balls $Q_{1}^{0}, Q_{2}^{0}, Q_{3}^{0}, \ldots, Q_{n}^{0} \in Q^{0}$ such that the following hold:

1. $Q_{i}^{0} \in \mathcal{Q}^{0}, 1 \leq i \leq n$;

2. $Q_{i}^{0} \cap Q_{j}^{0}=\varnothing, i \neq j, 1 \leq i, j \leq n$;

3. $W^{0} \subseteq \bigcup_{i=1}^{n} Q_{i}^{0 *}$.

By (1.3) there is a constant $c \geq 1$ such that

$$
\frac{1}{c} \leq \frac{\left|Q^{*}(s, t, x)\right|}{|Q(s, t, x)|} \leq c
$$


for all $s \geq 1$.

So

$$
\begin{aligned}
\frac{\left|W^{0}\right|}{\left|A_{k}\right|} & =\frac{\left|A_{k}\right|+\left|W^{0} \backslash A_{k}\right|}{\left|A_{k}\right|}=1+\frac{\left|W^{0} \backslash A_{k}\right|}{\left|A_{k}\right|} \\
& \geq 1+\frac{\left|W^{0} \backslash A_{\delta_{1}}\right|}{\left|W^{0}\right|} \geq 1+\frac{\left|W^{0} \backslash A_{\delta_{1}}\right|}{\left|\bigcup_{i=1}^{n} Q_{i}^{0 *}\right|} \\
& \geq 1+\frac{\left|W^{0} \backslash A_{\delta_{1}}\right|}{\sum_{i=1}^{n}\left|Q_{i}^{0 *}\right|} \geq 1+\frac{\left|W^{0} \backslash A_{\delta_{1}}\right|}{c \sum_{i=1}^{n}\left|Q_{i}^{0}\right|} \\
& \geq 1+\frac{\left|\bigcup_{i=1}^{n} Q_{i}^{0} \backslash A_{\delta_{1}}\right|}{c \sum_{i=1}^{n}\left|Q_{i}^{0}\right|}=1+\frac{\sum_{i=1}^{n}\left|Q_{i}^{0} \backslash A_{\delta_{1}}\right|}{c \sum_{i=1}^{n}\left|Q_{i}^{0}\right|} .
\end{aligned}
$$

Since $\left|Q^{i} \cap A_{\delta_{1}}\right|<\xi\left|Q_{i}^{0}\right|$, we have

$$
\begin{aligned}
\left|Q_{i}^{0} \backslash A_{\delta_{1}}\right| & =\left|Q_{i}^{0}\right|-\left|Q_{i}^{0} \cap A_{\delta_{1}}\right| \\
& \geq\left|Q_{i}^{0}\right|-\xi\left|Q_{i}^{0}\right|=(1-\xi)\left|Q_{i}^{0}\right| .
\end{aligned}
$$

Combining (3.25) and (3.26) we have that

$$
\begin{aligned}
\frac{\left|W^{0}\right|}{\left|A_{k}\right|} & \geq 1+\frac{\sum_{i=1}^{n}(1-\xi)\left|Q_{i}^{0}\right|}{c \sum_{i=1}^{n}\left|Q_{i}^{0}\right|} \\
& =1+\frac{1-\xi}{c}
\end{aligned}
$$

which proves the lemma.

3.6. Proof of Lemma 3.8. If $x \in U^{r}$, then we set

$$
W_{\mathbb{R} x}^{1}=W_{\mathbb{R}}^{1} \cap \mathbb{R} \times\{x\} \quad \text { and } \quad W_{\mathbb{R} x}^{2}=W_{\mathbb{R}}^{2} \cap \mathbb{R} \times\{x\}
$$

for $x \in U^{r}$.

It is enough to prove that

$$
\left|W_{\mathbb{R} x}^{1}\right| \leq \frac{(\sigma-1)^{2} \eta^{2}}{(\sigma-1)^{2} \eta^{2}-1}\left|W_{\mathbb{R} x}^{2}\right| .
$$

This follows from Lemma 2.2 in [29], page 157, by taking

$$
\kappa=\frac{16(\sigma-1)^{2} \eta^{2}}{16(\sigma-1)^{2} \eta^{2}-1}
$$

and by setting

$$
g\left(\left(t_{1}, t_{2}\right)\right)=\left(t_{2}-\kappa\left(t_{2}-t_{1}\right), t_{2}\right) .
$$


4. A first difference estimate. Repeated use of Proposition 1.6 yields the following:

THEOREM 4.1. There are $\gamma \in(0,1]$ and $c>0$ such that, for all $r \geq 1$, $z, x \in U$ and every function $u$ satisfying $\left(\partial_{1}+(I-\mu)\right) u=0$ in $\llbracket-r^{2}, 0 \rrbracket \times U^{r}$,

$$
\left|\partial_{z} u(0, x)\right| \leq c r^{-\gamma}\|u\|_{\infty}
$$

Combining this result with Varopoulos's theorem (Theorem 1.5) we have the following:

COROLLARY 4.2. There are $\gamma \in(0,1]$ and $c>0$ such that, for all $n \in \mathbb{N}$, $z \in U$

$$
\left\|\partial_{z} \mu^{* n}\right\|_{\infty} \leq c n^{-(D+\gamma) / 2}
$$

5. Results on the algebraic structure of $\boldsymbol{N}$. In this section we recall certain well-known results on the algebraic structure of $N$ (cf. [19, 21, 33, 45, 46, 50]).

5.1. The filtration of the Lie algebra. Let $\mathfrak{n}$ be the Lie algebra of $N$, which we identify with the left invariant vector fields on $N$.

We set $\mathfrak{n}_{1}=\mathfrak{n}$ and $\mathfrak{n}_{i+1}=\left[\mathfrak{n}_{1}, \mathfrak{n}_{i}\right], i \geq 1$. Since $\mathfrak{n}$ is nilpotent, we have the filtration

$$
\mathfrak{n}=\mathfrak{n}_{1} \supseteq \mathfrak{n}_{2} \supseteq \cdots \supseteq \mathfrak{n}_{m} \supseteq \mathfrak{n}_{m+1}=\{0\}, \quad \mathfrak{n}_{m} \neq\{0\} .
$$

We consider linear subspaces $\mathfrak{a}_{1}, \ldots, \mathfrak{a}_{m}$ of $\mathfrak{n}$ such that

$$
\mathfrak{n}_{i}=\mathfrak{a}_{i} \oplus \cdots \oplus \mathfrak{a}_{m}, \quad 1 \leq i \leq m .
$$

We set

$$
\begin{aligned}
n_{0} & =0, \quad n_{i}=\operatorname{dim}\left(\mathfrak{a}_{1} \oplus \cdots \oplus \mathfrak{a}_{i}\right), \quad 1 \leq i \leq m, \\
\sigma(j) & =i, \quad \text { for } n_{i-1}<j \leq n_{i}, \\
q & =n_{m}=\operatorname{dim}(\mathfrak{n}) .
\end{aligned}
$$

Notice that the homogeneous dimension $D$ of $N$ is given by

$$
D=\sigma(1)+\cdots+\sigma(q) .
$$

We consider a basis $\left\{X_{1}, \ldots, X_{q}\right\}$ of $\mathfrak{n}$ such that $\left\{X_{n_{i-1}+1}, \ldots, X_{n_{i}}\right\}$ is a basis of $\mathfrak{a}_{i}, 1 \leq i \leq m$.

On the linear space $\mathfrak{n}$, we define the Lie bracket $[\cdot, \cdot]_{0}$ by setting

$$
\left[X_{i}, X_{j}\right]_{0}=\operatorname{pr}_{\mathfrak{a}_{\sigma(i)+\sigma(j)}}\left[X_{i}, X_{j}\right] .
$$

We denote by $\mathfrak{n}_{0}$ the Lie algebra $\mathfrak{n}_{0}=\left(\mathfrak{n},[\cdot, \cdot]_{0}\right)$. Note that $\mathfrak{n}_{0}$ is nilpotent. 
5.2. Dilations and the exponential coordinates. Using the exponential coordinates of the second kind (or Malcev coordinates)

$$
\phi: \mathbb{R}^{q} \rightarrow N, \quad \phi: x=\left(x_{q}, \ldots, x_{1}\right) \rightarrow \exp x_{q} X_{q} \cdots \exp x_{1} X_{1}
$$

we identify $N$, as a differential manifold, with $\mathbb{R}^{q}$.

Let $\tau_{\varepsilon}, \varepsilon>0$, be the family of dilations of $N$ defined by

$$
\tau_{\varepsilon}:\left(x_{q}, \ldots, x_{1}\right) \rightarrow\left(\varepsilon^{\sigma(q)} x_{q}, \ldots, \varepsilon^{\sigma(1)} x_{1}\right) .
$$

Also, let $*_{\varepsilon}, \varepsilon>0$, be the family of group products defined by

$$
x *_{\varepsilon} y=\tau_{\varepsilon}\left[\left(\tau_{\varepsilon^{-1}} x\right)\left(\tau_{\varepsilon^{-1}} y\right)\right]
$$

and let

$$
x *_{0} y=\lim _{\varepsilon \rightarrow 0} x *_{\varepsilon} y .
$$

Then $N_{0}=\left(N, *_{0}\right)$ is a stratified nilpotent Lie group whose Lie algebra is isomorphic to $\mathfrak{n}_{0}$. We identify $\mathfrak{n}_{0}$ with the $*_{0}$-left invariant vector fields.

If $X \in \mathfrak{n}$ is a left invariant vector field on $N$, then we denote by $X_{0}$ the $*_{0}$-left invariant vector field satisfying $X_{0}(e)=X(e)$.

In particular we denote by $X_{0 i}$ the $*_{0}$-left invariant vector fields satisfying $X_{0 i}(0)=X_{i}(0), 1 \leq i \leq q$.

Note that

$$
X_{0 i}=\lim _{\varepsilon \rightarrow 0} \frac{1}{\varepsilon^{\sigma(i)}} d \tau_{\varepsilon}\left(X_{i}\right), \quad 1 \leq i \leq q,
$$

and that

$$
X_{0 i}=\frac{1}{\varepsilon^{\sigma(i)}} d \tau_{\varepsilon}\left(X_{0 i}\right), \quad 1 \leq i \leq q .
$$

We now give an expression of the left invariant vectors fields of $N$ as vector fields on $\mathbb{R}^{q}$.

If $X=a_{1} X_{1}+\cdots+a_{q} X_{q}$, then we set $\operatorname{pr}_{i}(X)=a_{i}, i=1, \ldots, q$.

We also denote by $\overline{\operatorname{ad}} X_{i}$ the linear transformations of $\mathfrak{n}$ defined by

$$
\overline{\operatorname{ad}}\left(X_{i}\right) X_{j}= \begin{cases}0, & \text { for } i \geq j, \\ \operatorname{ad}\left(X_{i}\right) X_{j}, & \text { for } i<j .\end{cases}
$$

LeMmA 5.1 (cf. [4]). Let $X$ be a left invariant vector field on $N$. Then $X(x)=a_{q}(x) \frac{\partial}{\partial x_{q}}+\cdots+a_{1}(x) \frac{\partial}{\partial x_{1}}$ with

$$
\begin{aligned}
a_{i}(x)=\operatorname{pr}_{i}\left[e^{x_{i-1} \overline{\mathrm{ad}} X_{i-1}} \cdots e^{x_{1} \overline{\mathrm{ad}} X_{1}}(X)\right] \\
=\operatorname{pr}_{i}\left[\sum_{\lambda_{1} \sigma(1)+\cdots+\lambda_{i-1} \sigma(i-1) \leq \sigma(i)-1} \frac{1}{\lambda_{1} !} \cdots \frac{1}{\lambda_{i-1} !} x_{1}^{\lambda_{1}} \cdots x_{i-1}^{\lambda_{i-1}}\right. \\
\times\left(\overline{\operatorname{ad}} X_{i-1}\right)^{\left.\lambda_{i-1} \cdots\left(\overline{\mathrm{ad}} X_{1}\right)^{\lambda_{1}}(X)\right] .}
\end{aligned}
$$


Note that if $X_{0}$ is the associated $*_{0}$-left invariant vector field satisfying $X_{0}(e)=$ $X(e)$, then $X_{0}(x)=a_{0 q}(x) \frac{\partial}{\partial x_{q}}+\cdots+a_{01}(x) \frac{\partial}{\partial x_{1}}$ with

$$
\begin{array}{r}
a_{0 i}(x)=\operatorname{pr}_{i}\left[\sum_{\lambda_{1} \sigma(1)+\cdots+\lambda_{i-1} \sigma(i-1)=\sigma(i)-1} \frac{1}{\lambda_{1} !} \cdots \frac{1}{\lambda_{i-1} !} x_{1}^{\lambda_{1}} \cdots x_{i-1}^{\lambda_{i-1}}\right. \\
\left.\times\left(\overline{\operatorname{ad}} X_{i-1}\right)^{\lambda_{i-1}} \cdots\left(\overline{\operatorname{ad}} X_{1}\right)^{\lambda_{1}}(X)\right] .
\end{array}
$$

Let us set, for $f \in C^{\infty}$ and $\ell \in \mathbb{N}$,

$$
\begin{aligned}
& \nabla^{\ell} f(x)=\sum_{\substack{a \leq \ell \\
\sigma\left(i_{1}\right)+\cdots+\sigma\left(i_{a}\right) \geq \ell}}\left|\frac{\partial}{\partial x_{i_{1}}} \cdots \frac{\partial}{\partial x_{i_{a}}} f(x)\right|, \\
& \nabla_{X}^{\ell} f(x)=\sum_{\substack{a \leq \ell \\
\sigma\left(i_{1}\right)+\cdots+\sigma\left(i_{a}\right) \geq \ell}}\left|X_{i_{1}} \cdots X_{i_{a}} f(x)\right| .
\end{aligned}
$$

Then it follows from (5.3) that there are $c>0$ and $k \in \mathbb{N}$ such that, for all $x \in N$ and $f \in C^{\infty}$,

$$
\frac{1}{c(1+|x|)^{k}} \nabla^{\ell} f(x) \leq \nabla_{X}^{\ell} f(x) \leq c(1+|x|)^{k} \nabla^{\ell} f(x) .
$$

\subsection{Taylor expansions.}

LEMMA 5.2. Let $f \in C^{\infty}$. Then

$$
\begin{array}{cc}
\frac{\partial}{\partial x_{i}} f(0)=X_{i} f(0), & 1 \leq i \leq q, \\
\frac{\partial}{\partial x_{i}} \frac{\partial}{\partial x_{j}} f(0)=X_{i} X_{j} f(0), & 1 \leq j \leq i \leq n_{1}
\end{array}
$$

and

$$
\begin{aligned}
\frac{\partial}{\partial x_{i}} \frac{\partial}{\partial x_{j}} f(0)= & X_{i} X_{j} f(0) \\
& -\sum_{n_{1}<\lambda \leq q}\left(\operatorname{pr}_{\lambda}\left[X_{i}, X_{j}\right]\right) X_{\lambda} f(0), \quad 1 \leq i<j \leq n_{1} .
\end{aligned}
$$

PROOF. Equation (5.6) follows immediately from (5.3). Equation (5.7) follows also from (5.3), since

$$
X_{i} X_{j} f(0)=\frac{\partial}{\partial y_{i}} X_{j} f(0)=\frac{\partial}{\partial x_{i}} \frac{\partial}{\partial x_{j}} f(0), \quad 1 \leq j \leq i \leq n_{1} .
$$


Finally, to prove (5.8) we observe that

$$
\frac{\partial}{\partial x_{i}} \frac{\partial}{\partial x_{j}} f(0)=\frac{\partial}{\partial x_{j}} \frac{\partial}{\partial x_{i}} f(0)
$$

and hence, by (5.6) and (5.7),

$$
\begin{aligned}
\frac{\partial}{\partial x_{i}} \frac{\partial}{\partial x_{j}} f(0) & =X_{j} X_{i} f(0) \\
& =\left(X_{i} X_{j}-\left[X_{i}, X_{j}\right]\right) f(0) \\
& =X_{i} X_{j} f(0)-\sum_{n_{1}<\lambda \leq q}\left(\operatorname{pr}_{\lambda}\left[X_{i}, X_{j}\right]\right) X_{\lambda} f(0) .
\end{aligned}
$$

Let us now assume that $V$ is a compact neighborhood of the identity element $e$ of $N$ which, viewed as subset of $\mathbb{R}^{q}$, is also convex. Also, let us denote by $P_{i}$ the monomial $p_{i}(x)=x_{i}, x=\left(x_{1}, \ldots, x_{q}\right) \in \mathbb{R}^{q}, 1 \leq i \leq q$.

LEMMA 5.3. Let $V$ be as above. Then there is a constant $c>0$ such that, for all $f \in C^{\infty}(N)$ and all $x \in N, y \in V$,

$$
f(x y)=f(x)+F_{x}(y) \quad \text { with }\left|F_{x}(y)\right| \leq c\left\|\nabla_{X} f\right\|_{L^{\infty}(x V)},
$$

$$
f(x y)=f(x)+\sum_{1 \leq i \leq n_{1}} P_{i}(y) X_{i} f(x)+F_{x}(y)
$$

with $\left|F_{x}(y)\right| \leq c\left\|\nabla_{X}^{2} f\right\|_{L^{\infty}(x V)}$ and

$$
\begin{aligned}
f(x y)= & f(x)+\sum_{1 \leq i \leq n_{1}} P_{i}(y) X_{i} f(x)+\frac{1}{2} \sum_{1 \leq i, j \leq n_{1}} P_{i}(y) P_{j}(y) X_{i} X_{j} f(x) \\
& +\sum_{\substack{n_{1}<i \leq n_{2}\\
}}\left(P_{i}(y)-\frac{1}{2} \sum_{1 \leq \lambda<\mu \leq n_{1}} P_{\lambda}(y) P_{\mu}(y) \operatorname{pr}_{i}\left[X_{\lambda}, X_{\mu}\right]\right) X_{i} f(x) \\
& +F_{x}(y)
\end{aligned}
$$

with $\left|F_{x}(y)\right| \leq c\left\|\nabla_{X}^{3} f\right\|_{L^{\infty}(x V)}$.

PROOF. We only give the proof of (5.11). The proofs of (5.9) and (5.10) are similar. Let $f^{\prime}(y)=f(x y)$. If $y \in V$, then by the Taylor formula (in $\mathbb{R}^{q}$ )

$$
\begin{aligned}
f^{\prime}(y)= & f^{\prime}(0)+\sum_{1 \leq i \leq q} P_{i}(y) \frac{\partial}{\partial y_{i}} f^{\prime}(0) \\
& +\frac{1}{2} \sum_{1 \leq i, j \leq q} P_{i}(y) P_{j}(y) \frac{\partial}{\partial y_{i}} \frac{\partial}{\partial y_{j}} f^{\prime}(0)+F^{\prime}(y)
\end{aligned}
$$




$$
\begin{aligned}
= & f^{\prime}(0)+\sum_{1 \leq i \leq n_{1}} P_{i}(y) \frac{\partial}{\partial y_{i}} f^{\prime}(0)+\frac{1}{2} \sum_{1 \leq i, j \leq n_{1}} P_{i}(y) P_{j}(y) \frac{\partial}{\partial y_{i}} \frac{\partial}{\partial y_{j}} f^{\prime}(0) \\
& +\sum_{n_{1}<i \leq n_{2}} P_{i}(y) \frac{\partial}{\partial y_{i}} f^{\prime}(0)+F^{\prime \prime}(y),
\end{aligned}
$$

where

$$
\left|F^{\prime}(y)\right| \leq c\left\|\nabla^{3} f^{\prime}\right\|_{L^{\infty}(V)}
$$

and hence also

$$
\left|F^{\prime \prime}(y)\right| \leq c\left\|\nabla^{3} f^{\prime}\right\|_{L^{\infty}(V)} .
$$

So, by Lemma 5.2,

$$
\begin{aligned}
f^{\prime}(y)= & f^{\prime}(0)+\sum_{1 \leq i \leq n_{1}} P_{i}(y) X_{i} f^{\prime}(0)+\frac{1}{2} \sum_{1 \leq i, j \leq n_{1}} P_{i}(y) P_{j}(y) X_{i} X_{j} f^{\prime}(0) \\
& +\sum_{n_{1}<i \leq q}\left(P_{i}(y)-\frac{1}{2} \sum_{1 \leq \lambda<\mu \leq n_{1}} P_{\lambda}(y) P_{\mu}(y) \operatorname{pr}_{i}\left[X_{\lambda}, X_{\mu}\right]\right) X_{i} f^{\prime}(0) \\
& +F^{\prime \prime}(y)
\end{aligned}
$$

If we set

$$
F(y)=F^{\prime \prime}(y)+\sum_{n_{2}<i \leq q}\left(P_{i}(y)-\frac{1}{2} \sum_{1 \leq \lambda<\mu \leq n_{1}} P_{\lambda}(y) P_{\mu}(y) \operatorname{pr}_{i}\left[X_{\lambda}, X_{\mu}\right]\right) X_{i} f^{\prime}(0)
$$

then, by (5.5),

$$
|F(y)| \leq c\left\|\nabla_{X}^{3} f^{\prime}\right\|_{L^{\infty}(V)}
$$

Also,

$$
\begin{aligned}
f^{\prime}(y)= & f^{\prime}(0)+\sum_{1 \leq i \leq n_{1}} P_{i}(y) X_{i} f^{\prime}(0)+\frac{1}{2} \sum_{1 \leq i, j \leq n_{1}} P_{i}(y) P_{j}(y) X_{i} X_{j} f(0) \\
& +\sum_{n_{1}<i \leq n_{2}}\left(P_{i}(y)-\frac{1}{2} \sum_{1 \leq \lambda<\mu \leq n_{1}} P_{\lambda}(y) P_{\mu}(y) \operatorname{pr}_{i}\left[X_{\lambda}, X_{\mu}\right]\right) X_{i} f^{\prime}(0) \\
& +F(y) .
\end{aligned}
$$

Given the left invariance of the vector fields $X_{i}$, this implies (5.11).

COROLlaRY 5.4. There is a constant $c>0$ such that, for all $f \in C^{\infty}(N)$ and all $x \in N, y \in V$, the following hold: 
(i) if $1 \leq v \leq n_{1}$, then

$$
X_{v} f(x y)=X_{v} f(x)+\sum_{1 \leq i \leq n_{1}} P_{i}(y) X_{i} X_{v} f(x)+F_{x}(y)
$$

with $\left|F_{x}(y)\right| \leq c\left\|\nabla_{X}^{3} f\right\|_{L^{\infty}(x V)}$;

(ii) if $n_{1}<v \leq n_{2}$,

$$
X_{v} f(x y)=X_{v} f(x)+F_{x}(y) \quad \text { with }\left|F_{x}(y)\right| \leq c\left\|\nabla_{X}^{3} f\right\|_{L^{\infty}(x V)} .
$$

6. Centered sub-Laplacians on $N$. Let $L=-\left(E_{1}^{2}+\cdots+E_{p}^{2}\right)+E_{0}$ be a left invariant sub-Laplacian on $N$ and let us assume that it is centered, that is, that $E_{0} \in[\mathfrak{n}, \mathfrak{n}]$.

Let $\left\{X_{1}, \ldots, X_{q}\right\}$ be the basis of $\mathfrak{n}$ introduced in Section 5. Since the vector fields $E_{i}$ are linear combinations of the vector fields $X_{i}$, the sub-Laplacian $L$ can also be written as

$$
L=-\sum_{1 \leq i, j \leq q} a_{i j} X_{i} X_{j}-\sum_{n_{1}<i \leq q} a_{i} X_{i} .
$$

Note that $a_{i j}=a_{j i}, 1 \leq i, j \leq q$. Also the assumption that the vector fields $E_{1}, \ldots, E_{p}$ satisfy Hörmander's condition implies that the $\left(n_{1} \times n_{1}\right)$ matrix $B=\left(b_{i j}\right)$ with entries $b_{i j}=a_{i j}, 1 \leq i, j \leq n_{1}$, is positive definite.

We associate with $L$ the limit (at $\infty$ ) sub-Laplacian

$$
L_{0}=\lim _{\varepsilon \rightarrow 0} \frac{1}{\varepsilon^{2}} d \tau_{\varepsilon}(L)=-\sum_{1 \leq i, j \leq n_{1}} a_{i j} X_{0 i} X_{0 j}-\sum_{n_{1}<i \leq n_{2}} a_{i} X_{0 i} .
$$

Note that $L_{0}$ is dilation invariant; that is, it satisfies

$$
L_{0}=\frac{1}{\varepsilon^{2}} d \tau_{\varepsilon}\left(L_{0}\right), \quad \varepsilon>0 .
$$

6.1. Polynomials. Since we have identified $N$, as a differential manifold, with $\mathbb{R}^{q}$ using the exponential coordinates of the second kind, the monomials on $\mathbb{R} \times N$ will just be monomials on $\mathbb{R} \times \mathbb{R}^{q}$.

Let $P(t, x)=t^{i_{0}} x_{1}^{i_{1}} \cdots x_{q}^{i_{q}}$ be such a monomial. Then the homogeneous degree $\operatorname{deg}_{H} P$ of $P$ is also given by

$$
\operatorname{deg}_{H} P=2 i_{0}+i_{1} \sigma(1)+\cdots+i_{q} \sigma(q) .
$$

By (5.3),

$$
\operatorname{deg}_{H}\left(\frac{\partial}{\partial t}+L\right) P(x) \leq \operatorname{deg}_{H} P(t, x)-2
$$

Also, by (5.4),

$$
\left(\frac{\partial}{\partial t}+L\right) P(t, x)=\left(\frac{\partial}{\partial t}+L_{0}\right) P(t, x)+Q(t, x),
$$


where $Q(t, x)$ is a polynomial satisfying

$$
\operatorname{deg}_{H} Q \leq \operatorname{deg}_{H} P-3 .
$$

Using induction on the dimension $q$ of the Lie algebra $\mathfrak{n}$ of $N$ and the homogeneous degree $\operatorname{deg}_{H} P$ of $P$ we can prove that there is a polynomial $Q(t, x)$ satisfying

$$
\begin{gathered}
\left(\frac{\partial}{\partial t}+L_{0}\right) Q(t, x)=P(t, x), \\
\operatorname{deg}_{H} Q(t, x)=\operatorname{deg}_{H} P(t, x)+2 .
\end{gathered}
$$

Combining (6.3), (6.4) and (6.5) we have the following:

LEMMA 6.1. With every monomial $P(t, x)$ as above we can associate a polynomial

$$
Q_{P}(t, x)=P(t, x)+W(t, x)
$$

satisfying

$$
\begin{aligned}
\operatorname{deg}_{H} W & \leq \operatorname{deg}_{H} P-1, \\
\left(\frac{\partial}{\partial t}+L_{0}\right) P(t, x) & =\left(\frac{\partial}{\partial t}+L\right) Q_{P}(t, x) .
\end{aligned}
$$

Note that the polynomial $Q_{P}(t, x)$ in the above lemma, is not necessarily unique.

From now on, for all $d \in \mathbb{N}$, we denote by

$$
P_{0}(t, x), P_{2}(t, x), \ldots, P_{v_{d}}(t, x)
$$

the monomials with homogeneous degree less than or equal to $d$. Given a centered left invariant sub-Laplacian $L$ on $N$ (in this article, this is either $L_{\mu}$ or $L_{H \mu}$ ), we associate with those monomials, polynomials

$$
Q_{P_{0}}(t, x), Q_{P_{2}}(t, x), \ldots, Q_{P_{v_{d}}}(t, x)
$$

satisfying (6.6).

Note that, for $0 \leq i \leq v_{2}$, we can take $Q_{P_{i}}=P_{i}$. Note also that $v_{0}=0$, $v_{1}=n_{1}$ and $v_{1}<n_{2}<v_{2}$. So, we assume that $P_{0}(t, x)=Q_{P_{0}}(t, x)=1$, that $P_{i}(t, x)=Q_{P_{i}}(t, x)=x_{i}$, for $1 \leq i \leq n_{2}$, and that $P_{i}=Q_{P_{i}}$, for $v_{1}<i \leq v_{2}$.

6.2. A Taylor formula for the heat functions. The following Taylor formula for the heat functions is proved in [7]. 
THEOREM 6.2. For all $n \in \mathbb{N}$ there is a $c_{n}>0$ such that for all $r, R \in \mathbb{N}$, $R \geq r \geq 1$, and all functions $u$ satisfying

$$
\left(\frac{\partial}{\partial t}+L\right) u=0 \quad \text { in }\left(-R^{2}, R^{2}\right) \times V^{R}
$$

we have

$$
\begin{aligned}
& \sup \left\{\left|u-\sum_{0 \leq i \leq v_{n}} A_{i} R^{-\operatorname{deg}_{H} P_{i}} Q_{P_{i}}\right| ;\left(r^{2}, r^{2}\right) \times V^{r}\right\} \\
& \quad<c_{n}\left(\frac{R}{r}\right)^{-(n+1)}\|u\|_{\infty},
\end{aligned}
$$

where the constants $A_{i}$ satisfy

$$
\left|A_{i}\right| \leq c_{n}\|u\|_{\infty}
$$

for all $0 \leq i \leq v_{n}$ and

$$
\left(\frac{\partial}{\partial t}+L\right)\left(\sum_{\nu_{d-1}<i \leq \nu_{d}} A_{i} Q_{P_{i}}\right)=0
$$

for all $1<k \leq n$.

\subsection{A Harnack inequality.}

THEOREM 6.3 [7]. Let $V$ be a compact connected neighborhood of the identity element $e$ of $N$, let $\alpha, a, \beta, b \in \mathbb{N}, 1 \leq \alpha<\alpha+a<\beta$, and let $k, \ell \in \mathbb{N}$. Then there is a $c \in \mathbb{N}$ such that for all $r \in \mathbb{N}, r \geq 1$, and all $u \geq 0$ satisfying

$$
\left(\frac{\partial}{\partial t}+L\right) u=0 \quad \text { in }\left(0,\left(\beta^{2}+b^{2}\right) r^{2}\right) \times V^{c r},
$$

we have

$$
\begin{aligned}
& \sup \left\{\left|\frac{\partial^{k}}{\partial t^{k}} X_{i_{1}} \cdots X_{i_{\ell}} u\right| ;\left(\alpha r^{2},\left(\alpha+a^{2}\right) r^{2}\right) \times V^{a r}\right\} \\
& \quad \leq c r^{-2 k-\sigma\left(i_{1}\right)-\cdots-\sigma\left(i_{\ell}\right)} \inf \left\{u ;\left(\beta r^{2},\left(\beta+b^{2}\right) r^{2}\right) \times V^{b r}\right\} .
\end{aligned}
$$

6.4. Estimates for the heat kernel. The heat kernel $p_{t}(x, y)$ of $L$ satisfies the following Gaussian estimate (cf. [7]).

THEOREM 6.4. There is a constant $c>0$ such that, for all $x, y \in N$ and $t \geq 1$,

$$
\frac{1}{c} t^{-D / 2} \exp \left(-c \frac{\left|x^{-1} y\right|_{N}^{2}}{t}\right) \leq p_{t}(x, y) \leq c t^{-D / 2} \exp \left(-\frac{\left|x^{-1} y\right|_{N}^{2}}{c t}\right) .
$$


Combining (6.9) with (6.8) we have the following:

COROLLARY 6.5. For all $k, \ell \in \mathbb{N}$ there is a constant $c>0$ such that

$$
\left|\frac{\delta^{k}}{\partial t^{k}} X_{i_{1}} \cdots X_{i_{n}} p_{t}(x, y)\right| \leq c t^{-\left(D+2 k+\sigma\left(i_{1}\right)+\cdots+\sigma\left(i_{\ell}\right)\right) / 2} \exp \left(-\frac{\left|x^{-1} y\right|_{N}^{2}}{c t}\right),
$$

for all $t \geq 1, x, y \in N$ and for all $1 \leq i_{j} \leq q, 1 \leq j \leq n$.

Let $p_{t}^{0}(x, y)$ be the heat kernel of $L_{0}$. Then we have the following analogue of the classical Berry-Esseen estimate (cf. [7, 20]):

THEOREM 6.6. There is a constant $c>0$ such that, for all $t \geq 1$ and $x \in N$,

$$
\left|p_{t}(x, e)-p_{t}^{0}(x, e)\right| \leq c t^{-(D+1) / 2} .
$$

It follows from (6.2) that

$$
p_{t}^{0}(x, y)=\varepsilon^{D} p_{\varepsilon^{2} t}^{0}\left(\tau_{\varepsilon} x, \tau_{\varepsilon} y\right), \quad \varepsilon>0,
$$

and hence there is a $C_{L_{0}}>0$ such that

$$
p_{t}^{0}(e, e)=C_{L_{0}} t^{-D / 2} .
$$

Combining (6.11) with (6.12) we have the following:

COROLLARY 6.7. There are constants $C_{L}>0$ and $c>0$ such that

$$
\left|p_{t}(x, e)-C_{L} t^{-D / 2}\right| \leq c t^{-(D+1) / 2}, \quad t \geq 1 .
$$

7. A smooth substitute for $|\boldsymbol{x}|_{N}$. The following proposition furnishes a positive smooth function $\rho(x)$ on a simply connected nilpotent Lie group $N$, which will replace $|x|_{N}$ in the proof of the Gaussian estimate (1.14). This function will be a convenient power of the Green function of a symmetric sub-Laplacian $L$ on $N$.

We use the notation of the previous section.

Proposition 7.1. There is a function $\rho(x) \in C^{\infty}(N)$ with the following properties: For all $n \in \mathbb{N}$ there is a constant $c \geq 1$ such that, for all $x \in N$ and all $1 \leq i_{j} \leq q, 1 \leq j \leq n$,

$$
\begin{array}{rlrl}
\rho(x) & \geq 0, \quad x \in N, & \\
\frac{1}{c}|x|_{N} & \leq \rho(x) \leq c|x|_{N} \quad \text { for }|x| \geq 2, \\
\left|X_{i_{1}} \cdots X_{i_{n}} \rho(x)\right| & \leq \frac{c}{|x|_{N}^{\sigma\left(i_{1}\right)+\cdots+\sigma\left(i_{n}\right)-1}} \quad \text { for }|x| \geq 2 .
\end{array}
$$


PROOF. If the homogeneous dimension $D$ of $N$ is $D \leq 2$, then $N$ is isomorphic either to $\mathbb{R}$ or to $\mathbb{R}^{2}$ and then we can take as $\rho$ the Euclidean norm. So let us assume that $D>2$. Let $L$ be a symmetric left invariant sub-Laplacian on $N$ and let $p_{t}(x, y)$ be its heat kernel. The Green function of $L$ is given by

$$
G_{L}(x, y)=\int_{0}^{\infty} p_{t}(x, y) d t
$$

Let $G_{L}(x)=G_{L}(x, e)$. Then it follows from (6.9) that there is a $c>0$ such that

$$
\frac{1}{c} \frac{1}{|x|_{N}^{D-2}} \leq G_{L}(x) \leq c \frac{1}{|x|_{N}^{D-2}}, \quad|x|_{N} \geq 2 .
$$

Since $L G_{L}(x)=0$, in $N \backslash\{e\}$ it follows from (6.10) that for all $n \in \mathbb{N}$ there is a $c>0$ such that

$$
\left|X_{i_{1}} X_{i_{2}} \cdots X_{i_{n}} G_{L}(x)\right| \leq \frac{c}{|x|_{N}^{D-2+\sigma\left(i_{1}\right)+\cdots+\sigma\left(i_{n}\right)}}, \quad|x|_{N} \geq 2,0 \leq \varepsilon \leq 1 .
$$

The function

$$
\rho(x)=\left(G_{L}(x)\right)^{-1 /(D-2)}
$$

satisfies (7.1).

8. Construction of the sub-Laplacian $L_{\mu}$ when $\Gamma$ is nilpotent. In this section we give the definition of the operator $L_{H \mu}$ when $\Gamma$ is nilpotent. If $\Gamma$ is not nilpotent, then the action of $\Gamma / \Gamma_{N}$ on $\Gamma_{N}$ gives rise to phenomena of homogenization and this makes the definition of $L_{H \mu}$ more complicated. To make this distinction, in the nilpotent case, we use the notation $L_{\mu}$ instead of $L_{H \mu}$.

We assume that $\Gamma=\Gamma_{N}$. If $\Gamma$ is nilpotent and the torsion subgroup $\tau(\Gamma)$ is not trivial, then we define $L_{\mu}$ to be the same as the operator $L_{\pi(\mu)}$ associated with the image $\pi(\mu)$ of $\mu$ under the quotient map $\pi: \Gamma \rightarrow \Gamma_{N}=\Gamma / \tau(\Gamma)$.

We use the notation of Sections 5 and 6.

The operator $L_{\mu}$ will be a centered sub-Laplcian which can be written as

$$
L_{\mu}=-\sum_{1 \leq i, j \leq n_{1}} a_{i j} X_{i} X_{j}-\sum_{n_{1}<i \leq n_{2}} a_{i} X_{i} .
$$

The coefficients $a_{i j}$ and $a_{i}$ are defined as follows.

The coefficients $a_{i j}$ are given by

$$
a_{i j}=\frac{1}{2} \sum_{x \in \Gamma} P_{i}(x) P_{j}(x) \mu(x), \quad 1 \leq i, j \leq n_{1} .
$$

Let

$$
b_{i}=\sum_{x \in \Gamma} P_{i}(x) \mu(x), \quad 1 \leq i \leq n_{2} .
$$


Note that since $\mu$ is centered $b_{i}=0,1 \leq i \leq n_{1}$.

The coefficients $a_{i}$ of $L_{\mu}$ are given by

$$
a_{i}=b_{i}-\frac{1}{2} \sum_{1 \leq \lambda<\mu \leq n_{1}} a_{\lambda \mu} \operatorname{pr}_{i}\left[X_{\lambda}, X_{\mu}\right], \quad n_{1}<i \leq n_{2} .
$$

Let $V$ be as in in Section 5.3 and let us assume that $V$ is large enough that $\operatorname{supp} \mu \subseteq V$.

The following lemma explains the relation between $\mu$ and $L_{\mu}$. It is an immediate consequence of Lemma 5.3.

LEMMA 8.1. There is a constant $c>0$ such that, for all functions $f \in$ $C^{\infty}(N)$ and all $x \in N$,

$$
|(I-\mu) f(x)| \leq c\left\|\nabla_{X}^{2} f\right\|_{L^{\infty}(x V)}
$$

and

$$
(I-\mu) f(x)=L_{\mu} f(x)+F(x),
$$

with

$$
|F(x)| \leq c\left\|\nabla_{X}^{3} f\right\|_{L^{\infty}(g V)} .
$$

COROLlary 8.2. There is a constant $c>0$ such that, for all functions $u(t, x) \in C^{\infty}(\mathbb{R} \times N)$ and all $x \in N$,

$$
\begin{aligned}
u(t+1, x)-\mu u(t, x) & =\left(\partial_{1}+(I-\mu)\right) u(t, x) \\
& =\left(\frac{\partial}{\partial t}+L_{\mu}\right) u(t, x)+F(t, x),
\end{aligned}
$$

where $F(t, x)$ satisfies

$$
|F(t, x)| \leq c\left\|\left|\frac{\partial^{2}}{\partial s^{2}} u\right|+\left|\nabla_{X}^{3} u\right|\right\|_{L_{([t, t+1] \times x V)}} .
$$

The following lemma asserts that $L_{\mu}$ is indeed a sub-Laplacian.

LEMMA 8.3. The $n_{1} \times n_{1}$ matrix $\left(a_{i j}\right)$ is positive definite.

PROOF. It is enough to prove that

$$
\sum_{1 \leq i, j \leq n_{1}} a_{i j} \xi_{i} \xi_{j}>0
$$

for all $\xi=\left(\xi_{1}, \ldots, \xi_{n_{1}}\right) \in \mathbb{R}^{n_{1}}, \xi \neq 0$. 
To this end, let us fix $\xi=\left(\xi_{1}, \ldots, \xi_{n_{1}}\right) \neq 0$ and consider the function

$$
\begin{aligned}
u(x) & =\left(\xi_{1} P_{1}(x)+\cdots+\xi_{n_{1}} P_{n_{1}}(x)\right)^{2} \\
& =\sum_{1 \leq i, j \leq n_{1}} \xi_{i} \xi_{j} P_{i}(x) P_{j}(x) .
\end{aligned}
$$

By (8.2),

$$
(I-\mu)\left(P_{i}(x) P_{j}(x)\right)=L_{\mu}\left(P_{i}(x) P_{j}(x)\right)=-\left(a_{i j}+a_{j i}\right)
$$

and hence

$$
(I-\mu) u(x)=2 \sum_{1 \leq i, j \leq n_{1}} a_{i j} \xi_{i} \xi_{j}
$$

If we had

$$
\sum_{1 \leq i, j \leq n_{1}} a_{i j} \xi_{i} \xi_{j}=0
$$

then we would have $(I-\mu) u=0$, that is, $\sum_{y \in \Gamma} u(x y) \mu(y)=u(x), x \in N$. Since $u(x)=0$, this would imply that $u(x)=0$ for all $x \in \Gamma_{N}$, which is false. Hence, (8.4) holds and the lemma follows.

9. Proof of Propositions 1.3 and 1.4 when $\Gamma$ is nilpotent. The goal of this section is to prove Propositions 1.3 and 1.4 when $\Gamma$ is nilpotent. Note that there is no loss of generality if we assume that the torsion subgroup $\tau(\Gamma)$ is trivial and hence that $\Gamma=\Gamma_{N}$.

We use the same notation $p_{t}^{\mu}(x, y)$ to denote both the heat kernel of the subLaplacian $L_{\mu}$ and its restriction to $\Gamma_{N}$.

The proofs are based on the following lemma.

LEMMA 9.1. There is a constant $c>0$ such that, for all $n \in \mathbb{N}, T \geq 1$,

$$
\left\|p_{n+T}^{\mu}-\mu^{n} p_{T}^{\mu}\right\|_{\infty} \leq c T^{-(D+1) / 2} .
$$

Proof. We have

$$
\begin{aligned}
p_{n+T}^{\mu}-\mu^{n} p_{T}^{\mu} & =p_{n+T}^{\mu}-\mu^{n-1} p_{1+T}^{\mu}+\mu^{n-1} p_{1+T}^{\mu}-\mu^{n} p_{T}^{\mu} \\
& =\sum_{0 \leq i \leq n-1} \mu^{i}\left(p_{n-i+T}^{\mu}-\mu p_{n-i-1+T}^{\mu}\right) .
\end{aligned}
$$

On the other hand, it follows from (8.3) that

$$
p_{t+1}^{\mu}-\mu p_{t}^{\mu}=\left(\frac{\partial}{\partial t}+L_{\mu}\right) p_{t}^{\mu}+V_{t}=V_{t}
$$


with $V_{t}$ satisfying

$$
\left|V_{t}(x, y)\right| \leq c_{1}\left\|\left|\frac{\partial^{2}}{\partial s^{2}} p_{s}^{\mu}(\cdot, y)\right|+\left|\nabla_{X}^{3} p_{s}^{\mu}(\cdot, y)\right|\right\|_{L_{([t, t+1] \times x V)}} .
$$

So, by (6.10) there is a $c>0$ such that

$$
\left\|p_{t+1}^{\mu}-\mu p_{t}^{\mu}\right\|_{\infty} \leq c t^{-(D+3) / 2}, \quad t \geq 1
$$

Combining (9.2) and (9.3) we have

$$
\begin{aligned}
\left\|p_{n+T}^{\mu}-\mu^{n} p_{T}^{\mu}\right\|_{\infty} & \leq \sum_{0 \leq i \leq n-1}\left\|\mu^{i}\right\|_{1}\left\|p_{n-i+T}^{\mu}(x, y)-\mu p_{n-i-1+T}^{\mu}\right\|_{\infty} \\
& \leq c \sum_{0 \leq i \leq n-1}(n-i-1+T)^{-(D+3) / 2} \\
& \leq c T^{-(D+1) / 2},
\end{aligned}
$$

which proves the lemma.

\subsection{Proof of Proposition 1.3. We have}

$$
\begin{aligned}
\sum_{y \in U^{r}} \mu^{n}(x, y) & \geq \frac{1}{\left\|p_{T}^{\mu}\right\|_{\infty}} \sum_{y \in U^{r}} \mu^{n}(x, y) p_{T}^{\mu}(y, e) \\
& =\frac{1}{\left\|p_{T}^{\mu}\right\|_{\infty}}\left(\mu^{n} p_{T}^{\mu}(x, e)-\sum_{\left\{y \notin U^{r}\right\}} \mu^{n}(x, y) p_{T}^{\mu}(y, e)\right) \\
& \geq \frac{1}{\left\|p_{T}^{\mu}\right\|_{\infty}}\left(p_{n+T}^{\mu}(x, e)-\left\|\mu^{n} p_{T}^{\mu}-p_{n+T}^{\mu}\right\|_{\infty}\right. \\
& \left.\quad-\sum_{\left\{y \notin U^{r}\right\}} \mu^{n}(x, y) p_{T}^{\mu}(y, e)\right) .
\end{aligned}
$$

Let us fix $a>1$. Then, by (6.9), there is a $c>1$ such that, for all $r, T \geq 1$,

$$
\inf \left\{p_{t+T}^{\mu}(x, e): x \in U^{a r}, a^{-2} r^{2} \leq t \leq a^{2} r^{2}\right\} \geq \frac{1}{c}\left(a^{2} r^{2}+T\right)^{-D / 2}
$$

and

$$
\sup \left\{p_{T}^{\mu}(x, e): x \notin U^{r}\right\} \leq c T^{-D / 2} \exp \left(-\frac{r^{2}}{c T}\right) .
$$

Also, by (9.1) there is a constant $c^{\prime}>0$ such that, for all $t, T \geq 1$,

$$
\left\|\mu^{n} p_{T}^{\mu}-p_{n+T}^{\mu}\right\|_{\infty} \leq c^{\prime} T^{-(D+1) / 2} .
$$


It follows that, for all $(n, x) \in \llbracket a^{-2} r^{2}, a^{2} r^{2} \rrbracket \times U^{a r}$,

$$
\begin{aligned}
& \sum_{y \in U^{r}} \mu^{n}(x, y) \\
& \quad \geq \frac{1}{c} T^{D / 2}\left(\frac{1}{c}\left(a^{2} r^{2}+T\right)^{-D / 2}-c^{\prime} T^{-(D+1) / 2}-c T^{-D / 2} \exp \left(-\frac{r^{2}}{c T}\right)\right) .
\end{aligned}
$$

If $T=\varepsilon r^{2}$, for some $\varepsilon \in(0,1)$, then we have

$$
\begin{aligned}
\sum_{y \in U^{r}} \mu^{n}(x, y) \geq & \frac{1}{c} \varepsilon^{D / 2}\left(\frac{1}{c}\left(a^{2}+\varepsilon\right)^{-D / 2}-c \varepsilon^{-(D+1) / 2} r^{-1}-c \varepsilon^{-D / 2} \exp \left(-\frac{1}{c \varepsilon}\right)\right) \\
\geq \frac{1}{c} \varepsilon^{D / 2}( & \frac{1}{c}\left(a^{2}+1\right)^{-D / 2} \\
& \left.-c \varepsilon^{-(D+1) / 2} r^{-1}-c \varepsilon^{-D / 2} \exp \left(-\frac{1}{c \varepsilon}\right)\right) .
\end{aligned}
$$

The proposition follows by choosing first $\varepsilon$ small enough and next $r$ large enough.

9.2. Proof of Proposition 1.4. Define $V$ as in Section 5.3, let $a_{1}>a_{2} \geq 1$ and set

$$
M=\sup \left\{\int_{V^{a_{2} r}} p_{r^{2}}^{\mu}(y, z) d z: y \notin U^{a_{1} r}\right\} .
$$

We have, for $r \in \mathbb{N}$,

$$
\begin{aligned}
\sum_{y \in U^{a_{1} r}} \mu^{r^{2}}(e, y) & \geq \sum_{y \in U^{a_{1} r}} \int_{V^{a_{2} r}} \mu^{r^{2}}(e, y) p_{r^{2}}^{\mu}(y, z) d z \\
& =\int_{V^{a_{2} r}} \mu^{r^{2}} p_{r^{2}}^{\mu}(e, z) d z-\sum_{\left\{y \notin U^{a_{1} r}\right\}} \int_{V^{a_{2} r}} \mu^{r^{2}}(e, y) p_{r^{2}}^{\mu}(y, z) d z \\
& \geq \int_{V^{a_{2} r}} \mu^{r^{2}} p_{r^{2}}^{\mu}(e, z) d z-M \\
& =\int_{V^{a_{2} r}} p_{2 r^{2}}^{\mu}(e, z) d z+\int_{V^{a_{2} r}}\left(\mu^{r^{2}} p_{r^{2}}^{\mu}-p_{2 r^{2}}^{\mu}(e, z) d z-M\right. \\
& \geq \int_{V^{a_{2} r}} p_{2 r^{2}}^{\mu}(e, z) d z-\left\|\mu^{r^{2}} p_{r^{2}}^{\mu}-p_{2 r^{2}}^{\mu}\right\|_{\infty}\left|V^{a_{2} r}\right|-M .
\end{aligned}
$$

Now, by (9.1) there is a $c_{1}>0$ such that, for all $r \in \mathbb{N}$,

$$
\left\|\mu^{r^{2}} p_{r^{2}}^{\mu}-p_{2 r^{2}}^{\mu}\right\|_{\infty} \leq c_{1} r^{-D-1} .
$$

Also, there is a $c_{2}>0$ such that

$$
\left|V^{r}\right| \leq c_{2} r^{D}, \quad r \in \mathbb{N} .
$$


Combining (9.4), (9.5) and (9.6) we have

$$
\sum_{y \in U^{a_{1} r}} \mu^{r^{2}}(e, y) \geq \int_{V^{a_{2} r}} p_{2 r^{2}}^{\mu}(e, z) d z-c_{1} c_{2} a_{2}^{D} r^{-1}-M .
$$

Now let us fix $\delta>0$ and let us chose $a_{2}>1$ such that, for all $r \geq 1$,

$$
\int_{V^{a_{2} r}} p_{2 r^{2}}^{\mu}(e, z) d z \geq 1-\frac{\delta}{3} .
$$

Let us choose $a_{1}>a_{2}$ so that $M \leq \partial / 3$ and $r_{0} \geq 1$ so that

$$
c_{1} c^{2} a_{2}^{D} r_{0}^{-1} \leq \frac{\delta}{3}
$$

Then it follows from (9.7) that

$$
\sum_{y \in U^{a_{1} r}} \mu^{r^{2}}(e, y) \geq 1-\delta
$$

and the proposition follows.

10. The proof of the Gaussian estimate when $\Gamma$ is nilpotent. In this section we give the proof of the Gaussian estimate (1.14) when the group $\Gamma$ is nilpotent. Note that there is no loss of generality if we assume that the torsion subgroup $\tau(\Gamma)$ is trivial and hence that $\Gamma=\Gamma_{N}$.

10.1. The functions $\rho_{k}, k \geq 1$. Let $\rho(x)$ be as in Section 7 and let the family of dilations $\tau_{\varepsilon}, \varepsilon>0$, be as in Section 5.2.

Let $V$ be a compact neighborhood of the identity element $e$ of $N$, as in Section 5.3, and let $|\cdot|_{N}$ be defined as in (1.7). Then there is a $C>1$ such that

$$
\tau_{1 / C k} V^{k} \subseteq V, \quad k \in \mathbb{N} .
$$

Let $0 \leq \phi \in C^{\infty}(N)$, such that

$$
\phi(x)= \begin{cases}0, & \text { for }|x|_{N} \leq 1, \\ 1, & \text { for }|x|_{N} \geq 4\end{cases}
$$

and set

$$
\phi_{k}(x)=\phi\left(\tau_{1 / C \sqrt{k}} x\right), \quad k \geq 1 .
$$

Then

$$
\phi_{k}(x)=0, \quad|x|_{N} \leq \sqrt{k},
$$

and there is a constant $\zeta>0$ such that

$$
\phi_{k}(x)=1, \quad|x|_{N} \geq \zeta \sqrt{k} .
$$


Also, if $\left\{X_{1}, \ldots, X_{q}\right\}$ is the basis of $\mathfrak{n}$ introduced in Section 5.1, then for every $n \in \mathbb{N}$ there is a constant $c>0$ such that

$$
\left|X_{i_{1}} X_{i_{2}} \cdots X_{i_{n}} \phi_{k}(x)\right| \leq c k^{-\left(\sigma\left(i_{1}\right)+\cdots+\sigma\left(i_{n}\right)\right) / 2}
$$

for all $x \in N$ and $1 \leq i_{j} \leq q, 1 \leq j \leq n$.

We set

$$
\rho_{k}(x)=\phi_{k}(x) \rho(x) \text {. }
$$

In the next lemma we gather the properties of the functions $\rho_{k}(x)$ that we need in the proof of Gaussian estimate (1.14). These properties are immediate consequences of (7.1).

LEMMA 10.1. For all $n \in \mathbb{N}$ there is a constant $c \geq 1$ such that, for all $k \geq 1$ and all $1 \leq i_{j} \leq q, 1 \leq j \leq n$,

$$
\begin{aligned}
\rho_{k}(x) & \geq 0, \quad x \in N, \\
\rho_{k}(x) & =0 \quad \text { for }|x|_{N} \leq \sqrt{k}, \\
\frac{1}{c}|x|_{N} & \leq \rho_{k}(x) \leq c|x|_{N} \quad \text { for }|x| \geq \zeta \sqrt{k}, \\
\left|X_{i_{1}} \cdots X_{i_{n}} \rho_{k}(x)\right| & \leq \frac{c}{|x|_{N}^{\sigma\left(i_{1}\right)+\cdots+\sigma\left(i_{n}\right)-1},}, \quad x \in N .
\end{aligned}
$$

10.2. The functions $H_{k}, k \geq 1$. For fixed constants $A>0$ and $B>0$ we consider the family of functions $H_{k}, k \geq 1$, defined by

$$
H_{k}(t, x)=\exp \left(-\frac{\left(\rho_{k}(x)+B \sqrt{k}\right)^{2}}{A(k+t)}\right), \quad t \geq 0, x \in N .
$$

LEMMA 10.2. There are constants $A>0$ and $B>0$ such that

$$
H_{k}(t+1, x)>\mu H_{k}(t, x)
$$

for all $(t, x) \in[0, k] \times N$ and $k \geq 1$.

ProOF. We observe that

$$
H_{k}(t+1, x)-\mu H_{k}(t, x)=H_{k}(t+1, x)-H_{k}(t, x)+(I-\mu) H_{k}(t, x)
$$

and that

$$
H_{k}(t+1, x)-H_{k}(t, x) \geq \inf _{t \leq s \leq t+1} \frac{\partial}{\partial s} H_{k}(s, x) .
$$

Let $V$ be a compact neighborhood of $e$, as in Section 5.3, and let us assume that supp $\mu \subseteq V$. Then, by Lemma 8.1,

$$
\left|(I-\mu) H_{k}(t, x)\right| \leq c \sup _{y \in x V}\left|\nabla_{X}^{2} H_{k}(t, y)\right| .
$$


We have

$$
\frac{\partial}{\partial t} H_{k}(t, x)=\frac{1}{A} \frac{1}{k+t} \frac{\left(\rho_{k}(x)+B \sqrt{k}\right)^{2}}{k+t} H_{k}(t, x) .
$$

Also, for all $X, Y \in \mathfrak{n}$,

$$
\begin{aligned}
Y H_{k}(t, x)= & -\frac{1}{A} \frac{1}{k+t} 2\left(\rho_{k}(x)+B \sqrt{k}\right) Y \rho_{k}(x) H_{k}(t, x), \\
X Y H_{k}(t, x)= & -\frac{1}{A} \frac{1}{k+t} 2 X \rho_{k}(x) Y \rho_{k}(x) H_{k}(t, x) \\
& -\frac{1}{A} \frac{1}{k+t} 2\left(\rho_{k}(x)+B \sqrt{k}\right) X Y \rho_{k}(x) H_{k}(t, x) \\
& +\frac{1}{A^{2}} \frac{1}{(k+t)^{2}} 4\left(\rho_{k}(x)+B \sqrt{k}\right)^{2} X \rho_{k}(x) Y \rho(x) H_{k}(t, x) .
\end{aligned}
$$

Case I $\left(|x|_{N} \leq \sqrt{k}-1\right.$ and $\left.t \in[0, k]\right)$. By construction, for all $|x|_{N} \leq \sqrt{k}$,

$$
H_{k}(t, x)=\exp \left(-\frac{B^{2} k}{A(k+t)}\right) \text {. }
$$

Hence, for all $|x|_{N} \leq \sqrt{k}-1$,

$$
H_{k}(t+1, x)>H_{k}(t, x)=\left(\mu H_{k+1}(t, \cdot)\right)(x) .
$$

Case II $\left(\sqrt{k}-1 \leq|x|_{N} \leq \zeta \sqrt{k}+1\right.$ and $\left.0 \leq t \leq k\right)$. By (10.7) and (10.9) there is a $c_{1}>0$ such that

$$
\begin{aligned}
H_{k}(t+1, x)-H_{k}(t, x) & \geq \frac{1}{A} \frac{1}{k+t+1} \frac{B^{2} k}{k+t+1} H_{k}(t, x) \\
& \geq \frac{1}{A} \frac{1}{k+t} c_{1} \frac{B^{2}}{2} H_{k}(t, x) .
\end{aligned}
$$

Also, by (10.8) and (10.10) there is a constant $c_{2}>0$ such that

$$
\begin{array}{r}
\left|(I-\mu) H_{k}(t, x)\right| \leq \frac{1}{A} \frac{1}{k+t}\left[\left(c_{2} \sqrt{k}+B \sqrt{k}\right) c_{2} \frac{1}{\sqrt{k}}+c_{2}\right. \\
+\left(c_{2} \sqrt{k}+B \sqrt{k}\right) \frac{1}{\sqrt{k}} \\
\left.+\frac{1}{A} \frac{1}{k}\left(c_{2} \sqrt{k}+B \sqrt{k}\right)^{2}\right] H_{k}(t, x) \\
\leq \frac{1}{A} \frac{1}{k+t}\left[c_{2}\left(c_{2}+B\right)+c_{2}+\left(c_{2}+B\right)\right. \\
\left.+\frac{1}{A}\left(c_{2}+B\right)^{2}\right] H_{k}(t, x) .
\end{array}
$$


Hence

$$
\begin{aligned}
H_{k}(t & +1, x)-\mu H_{k}(t, x) \\
& \geq \frac{1}{A} \frac{1}{k+t}\left[c_{1} \frac{B^{2}}{2}-c_{2}\left(c_{2}+B\right)-c_{2}-\left(c_{2}+B\right)-\frac{1}{A}\left(c_{2}+B\right)^{2}\right] H_{k}(t, x) .
\end{aligned}
$$

So, by choosing $B$ large enough that

$$
c_{1} \frac{B^{2}}{4}>c_{2}+c_{2}\left(c_{2}+B\right)+c_{2}\left(c_{2}+B\right)
$$

and $A$ large enough that

$$
c_{1} \frac{B^{2}}{4}>\frac{1}{A}\left(c_{2}+B\right)^{2}
$$

we have

$$
H_{k}(t+1, x)>\mu H_{k}(t, x) .
$$

Case III $\left(|x|_{N}>\zeta \sqrt{k}+1\right.$ and $\left.0 \leq t \leq k\right)$. By (10.7) and (10.9), there is a $c_{1}>0$ such that

$$
H_{k}(t+1, x)-H_{k}(t, x) \geq \frac{1}{A} \frac{1}{k+t} \frac{\left(c_{1}|x|_{N}+B \sqrt{k}\right)^{2}}{2 k} H_{k}(t, x) .
$$

Also, by (10.8) and (10.10) there is a $c_{2}>0$ such that

$$
\begin{aligned}
& \left|(I-\mu) H_{k}(t, x)\right| \\
& \leq \frac{1}{A} \frac{1}{k+t}\left[c_{2}\left(c_{2}|x|_{N}+B \sqrt{k}\right) \frac{1}{|x|_{N}}+c_{2}+c_{2}\left(c_{2}|x|_{N}+B \sqrt{k}\right) \frac{1}{|x|_{N}}\right. \\
& \left.+c_{2} \frac{1}{A} \frac{1}{k}\left(c_{2}|x|_{N}+B \sqrt{k}\right)^{2}\right] H_{k}(t, x) \\
& =\frac{1}{A} \frac{1}{k+t}\left[c_{2}^{2}+c_{2} B \frac{\sqrt{k}}{|x|_{N}} c_{2}+c_{2}^{2}+c_{2} B \frac{\sqrt{k}}{|x|_{N}}\right. \\
& \left.+c_{2} \frac{1}{A} \frac{\left(c_{2}|x|_{N}+B \sqrt{k}\right)^{2}}{k}\right] H_{k}(t, x) \\
& \leq \frac{1}{A} \frac{1}{k+t}\left[c_{2}^{2}+c_{2}^{2} B+c_{2}^{2}+c_{2} B+c_{2} \frac{1}{A} \frac{\left(c_{2}|x|_{N}+B \sqrt{k}\right)^{2}}{k}\right] H_{k}(t, x) .
\end{aligned}
$$

Hence

$$
\begin{aligned}
& H_{k}(t+1, x)-\mu H_{k}(t, x) \\
& \begin{array}{l}
\geq \frac{1}{A} \frac{1}{k+t}\left[\frac{\left(c_{1}|x|_{N}+B \sqrt{k}\right)^{2}}{2 k}-c_{2}^{2}-c_{2}^{2} B-c_{2}^{2}-c_{2} B\right. \\
\left.-c_{2} \frac{1}{A} \frac{\left(c_{2}|x|_{N}+B \sqrt{k}\right)^{2}}{k}\right] H_{k}(t, x) .
\end{array}
\end{aligned}
$$


So, by choosing $B$ large enough that

$$
\frac{\left(c_{1}|x|_{N}+B \sqrt{k}\right)^{2}}{4 k}>c_{2}+c_{2}^{2}+c_{2}+B c_{2}^{2}+c_{2} B
$$

and $A$ large enough that

$$
\frac{1}{4}\left(c_{1}|x|_{N}+B \sqrt{k}\right)^{2}>c_{2} \frac{1}{A}\left(c_{2}|x|_{N}+B \sqrt{k}\right)^{2}
$$

we have

$$
H_{k}(t+1, x)>\mu H_{k}(t, x) .
$$

10.3. Proof of Theorem 1.8. It is enough to prove that there is a $c>0$ such that

$$
\mu^{n}(x, e) \leq c n^{-D / 2} \exp \left(-\frac{|x|_{N}^{2}}{c n}\right), \quad n \geq 1, x \in N .
$$

Let us fix constants $A>0$ and $B>0$ such that the family of functions

$$
H_{k}(t, x)=\exp \left(-\frac{\left(\rho_{k}(x)+B \sqrt{k}\right)^{2}}{A(k+t)}\right), \quad k \geq 1,
$$

satisfy (10.6).

Let us consider the function

$$
u(n, x)=\sum_{y \in U^{\sqrt{k}}} \mu^{n}(x, y), \quad x \in \Gamma_{N}, n \in \mathbb{N} .
$$

Let us also fix a constant $C>0$ such that

$$
C H_{k}(0, x)>1, \quad|x|_{N} \leq 3 \sqrt{k},
$$

and consider the function

$$
F(n, x)=C H_{k}(n, x)-u(n, x) .
$$

Then $F(n, x)$ satisfies

$$
\begin{aligned}
F(n+1, x) & >\mu F(n, x), \quad x \in \Gamma_{N}, n \in[0, k] \\
F(0, x) & >0, \quad x \in \Gamma_{N},
\end{aligned}
$$

and hence

$$
F(t, x)>0, \quad t \in[0, k], x \in \Gamma_{N} .
$$


It follows that, for all $x \in \Gamma_{N}$ and $k \geq 1$,

$$
\sum_{y \in U^{\sqrt{k}}} \mu^{k}(x, y) \leq C \exp \left(-\frac{\left(\rho_{k}(x)+B \sqrt{k}\right)^{2}}{2 A k}\right) .
$$

On the other hand, it follows from (1.9) that there are $\beta \in \mathbb{N}$ and $\lambda>0$ such that, for all $x \in \Gamma_{N}$ and $n \in \mathbb{N}$,

$$
\mu^{n}(x, e) \leq \lambda \inf \left\{\mu^{\beta n}(x, y), y \in U^{\sqrt{n}}\right\} .
$$

Since $\left|U^{n}\right| \leq c n^{D}, n \in \mathbb{N}$, we have that

$$
\begin{aligned}
\mu^{n}(x, e) & \leq \lambda \frac{1}{\left|U^{\sqrt{n}}\right|} \sum_{y \in U^{\sqrt{n}}} \mu^{\beta n}(x, y) \\
& \leq \lambda c n^{-D / 2} C \exp \left(-\frac{\left(\rho_{\beta n}(x)+B \sqrt{\beta n}\right)^{2}}{2 A \beta n}\right)
\end{aligned}
$$

for all $x \in \Gamma_{N}$ and $n \geq 1$. This proves (10.11) and the theorem follows.

11. The proof of the Berry-Esseen estimate when $\Gamma$ is nilpotent. In this section we assume that $\Gamma=\Gamma_{N}$. If $\Gamma$ is nilpotent and the torsion subgroup $\tau(\Gamma)$ is not trivial, then we can just extend the different kernels from $\Gamma_{N}=\Gamma / \tau(\Gamma)$ to $\Gamma$, as explained in Section 1.4, and then the proofs remain exactly the same.

Let $L_{\mu}$ be the centered left invariant sub-Laplacian associated with $\mu$ and let $p_{t}^{\mu}(x, y)$ be its heat kernel $L_{\mu}$.

By (4.2) there are $\gamma \in(0,1]$ and $c>0$ such that

$$
\left\|\nabla_{U} \mu^{n}\right\|_{\infty} \leq c n^{-(D+\gamma) / 2}, \quad n \in \mathbb{N} .
$$

In this section we prove the following Berry-Esseen estimate (cf. [20, 34]):

THEOREM 11.1. There is a $c>1$ such that, for all $x, y \in \Gamma_{N}$ and $n \in \mathbb{N}$,

$$
\left|\mu^{n}(x, y)-p_{n}^{\mu}(x, y)\right| \leq c n^{-(D+\gamma) / 2} \text {. }
$$

Once we have proved Theorem 1.14, then (11.1) and hence (11.2) will hold with $\gamma=1$.

For the case when $\mu$ is symmetric, the above result was proved for $\gamma=1$ in [2]. We give below an adaptation of that proof.

Let $\Omega$ be a fundametal domain for $\Gamma_{N}$ (see Section 1.3) and let

$$
S_{t}(x, y)=\int_{\Omega} p_{t}^{\mu}(x h, y) d h, \quad x, y \in \Gamma_{N} .
$$

The proof of (11.2) is based on the following two lemmas, which are inspired by [14] (see also [7, 41]). 
LEMMA 11.2. There are constants $a, b \geq 1$ such that, for all $T \geq 1$ and $n \in \mathbb{N}$,

$$
\left\|\mu^{n}-p_{n}^{\mu}\right\|_{\infty} \leq a\left\|\left(\mu^{n}-p_{n}^{\mu}\right) S_{T}\right\|_{\infty}+b \sqrt{T} n^{-(D+\gamma) / 2} .
$$

LEMMA 11.3. There is a constant $c \geq 1$ such that if, for some $n \in \mathbb{N}$,

$$
\left\|\mu^{k}-p_{k}^{\mu}\right\|_{\infty} \leq A k^{-(D+\gamma) / 2} \quad \text { for all } 1 \leq k \leq n-1,
$$

then

$$
\left\|\left(\mu^{n}-p_{n}^{\mu}\right) S_{T}\right\|_{\infty} \leq c\left(1+\frac{A}{\sqrt{T}}\right) n^{-(D+\gamma) / 2} .
$$

PROOF OF THEOREM 11.1. If

$$
\left\|\mu^{k}-p_{k}^{\mu}\right\|_{\infty} \leq A k^{-(D+\gamma) / 2}, \quad 1 \leq k \leq n-1,
$$

then by (11.3) and (11.5),

$$
\begin{aligned}
\left\|\mu^{n}-p_{n}^{\mu}\right\|_{\infty} & \leq \alpha c\left(1+\frac{A}{\sqrt{T}}\right) n^{-(D+\gamma) / 2}+b \sqrt{T} n^{-(D+\gamma) / 2} \\
& \leq\left(a c+a c \frac{A}{\sqrt{T}}+b \sqrt{T}\right) n^{-(D+\gamma) / 2}
\end{aligned}
$$

So (11.2) can be proved by induction provided that for all $A$ large enough there is a $T \geq 1$ such that

$$
a c+a c \frac{A}{\sqrt{T}}+b \sqrt{T} \leq A .
$$

To this end, let us consider the function

$$
\varphi(x)=a c+a c A \frac{1}{x}+b x
$$

(note that $a, b, c \geq 1$ ) and take

$$
A \geq 9 a^{2} b^{2} c^{2} \quad \text { and } \quad T=\frac{a c A}{b} .
$$

Then we have

$$
\varphi(\sqrt{T}) \leq A
$$

This proves (11.6) and the theorem follows. 
11.1. Proof of Lemma 11.2. Let us set

$$
H_{n}(x, y)=\mu^{n}(x, y)-p_{n}^{\mu}(x, y)
$$

and assume that

$$
-\left\|H_{n}\right\|_{\infty}=\min \left\{H_{n}(x, y), x, y \in \Gamma_{N}\right\}
$$

(the case $\left\|H_{n}\right\|_{\infty}=\max \left\{H_{n}(x, y), x, y \in \Gamma_{N}\right\}$ can be treated in the same way). There are $x_{0}, y_{0} \in \Gamma_{N}$ such that

$$
H_{n}\left(x_{0}, y_{0}\right)=-\left\|H_{n}\right\|_{\infty} .
$$

Then

$$
\begin{aligned}
-\left\|H_{n} S_{T}\right\|_{\infty} \leq & \sum_{z \in \Gamma_{N}} H_{n}\left(x_{0}, z\right) S_{T}\left(z, y_{0}\right) \\
= & H_{n}\left(x_{0}, y_{0}\right) \sum_{\left|y_{0}^{-1} z\right|_{\Gamma_{N}} \leq c \sqrt{T}} S_{T}\left(z, y_{0}\right) \\
& +\sum_{\left|y_{0}^{-1} z\right|_{\Gamma_{N}} \leq c \sqrt{T}}\left[H_{n}\left(x_{0}, z\right)-H_{n}\left(x_{0}, y_{0}\right)\right] S_{T}\left(z, y_{0}\right) \\
& +\sum_{\left|y_{0}^{-1} z\right|_{\Gamma_{N}} \geq c \sqrt{T}} H_{n}\left(x_{0}, z\right) S_{T}\left(z, y_{0}\right) \\
\leq & -\left\|H_{n}\right\|_{\infty} \sum_{\left|y_{0}^{-1} z\right|_{\Gamma_{N}} \leq c \sqrt{T}} S_{T}\left(z, y_{0}\right) \\
& +c \sqrt{T}\left\|\nabla_{U} H_{n}\left(x_{0}, \cdot\right)\right\|_{\infty} \sum \\
& +\left\|H_{n}\right\|_{\infty} \sum_{\left|y_{0}^{-1} z\right|_{\Gamma_{N}} \geq c \sqrt{T}} S_{T}\left(z,\left.y_{0}^{-1} z\right|_{\Gamma_{N}} \leq c \sqrt{T}\right) .
\end{aligned}
$$

Hence, if

$$
\lambda=\sum_{\left|y_{0}^{-1} z\right|_{\Gamma_{N}} \leq c \sqrt{T}} S_{T}\left(z, y_{0}\right),
$$

then

$$
-\left\|H_{n} S_{T}\right\|_{\infty} \leq-\left\|H_{n}\right\|_{\infty} \lambda+c \sqrt{T} \lambda n^{-(D+\gamma) / 2}+\left\|H_{n}\right\|_{\infty}(1-\lambda),
$$

or

$$
(2 \lambda-1)\left\|H_{n}\right\|_{\infty} \leq\left\|H_{n} S_{T}\right\|_{\infty}+c \lambda \sqrt{T} \lambda n^{-(D+\gamma) / 2} .
$$

By choosing $c$ large enough, so that $\lambda>1 / 2$, we get

$$
\left\|H_{n}\right\|_{\infty} \leq \frac{1}{2 \lambda-1}\left\|H_{n} S_{T}\right\|_{\infty}+\frac{c \lambda}{2 \lambda-1} \sqrt{T} n^{-(D+\gamma) / 2},
$$

which proves the lemma. 
11.2. Proof of Lemma 11.3. It follows from (6.10) that there is a $c>0$ such that, for all $x, y \in \Gamma_{N}$ and $t \geq 1$,

$$
\left|p_{t+T}^{\mu}(x, y)-p_{t}^{\mu} S_{T}(x, y)\right| \leq c t^{-(D+1) / 2} .
$$

So, it is enough to prove that

$$
\left\|\mu^{n} S_{T}-p_{n+T}^{\mu}\right\|_{\infty} \leq c\left(1+\frac{A}{\sqrt{T}}\right) n^{-(D+\gamma) / 2} .
$$

We have

$$
\begin{aligned}
p_{n+T}^{\mu}-\mu^{n} S_{T}= & p_{n+T}^{\mu}-\mu^{n-1} p_{1+T}^{\mu}+\mu^{n-1} p_{1+T}^{\mu}-\mu^{n} S_{T} \\
= & \sum_{0 \leq i \leq n-2} \mu^{i}\left(p_{n-i+T}^{\mu}-\mu p_{n-i-1+T}^{\mu}\right)+\mu^{n-1}\left(p_{1+T}^{\mu}-\mu S_{T}\right) \\
= & \sum_{0 \leq i \leq n / 2} \mu^{i}\left(p_{n-i+T}^{\mu}-\mu p_{n-i-1+T}^{\mu}\right) \\
& +\sum_{n / 2<i \leq n-2}\left(\mu^{i}-p_{i}^{\mu}\right)\left(p_{n-i+T}^{\mu}-\mu p_{n-i-1+T}^{\mu}\right) \\
& +\left(\mu^{n-1}-p_{n-1}^{\mu}\right)\left(p_{1+T}^{\mu}-\mu S_{T}\right) \\
& +\sum_{n / 2<i \leq n-2} p_{i}^{\mu}\left(p_{n-i+T}^{\mu}-\mu p_{n-i-1+T}^{\mu}\right) \\
& +p_{n-1}^{\mu}\left(p_{1+T}^{\mu}-\mu S_{T}\right) .
\end{aligned}
$$

Now, by (6.10) and (8.3),

$$
\begin{aligned}
& \sum_{0 \leq i \leq n / 2}\left\|\mu^{i}\right\|_{1}\left\|p_{n-i+T}^{\mu}-\mu p_{n-i-1+T}^{\mu}\right\|_{\infty} \\
& \quad \leq c \sum_{0 \leq i \leq n / 2}(n-i-1+T)^{-(D+3) / 2} \\
& \quad \leq c\left(\frac{n}{2}+T\right)^{-(D+1) / 2} \\
& \quad \leq c n^{-(D+1) / 2} .
\end{aligned}
$$

By the induction hypothesis (11.4),

$$
\begin{aligned}
& \sum_{n / 2<i \leq n-2}\left\|\mu^{i}-p_{i}^{\mu}\right\|_{\infty}\left\|p_{n-i+T}^{\mu}-\mu p_{n-i-1+T}^{\mu}\right\|_{1} \\
& \quad \leq \sum_{n / 2<i \leq n-2} A i^{-(D+\gamma / 2}(n-i-1+T)^{-3 / 2} \\
& \quad \leq c A \frac{1}{\sqrt{T}} n^{-(D+\gamma) / 2} .
\end{aligned}
$$


To estimate the term $p_{1+T}^{\mu}-\mu S_{T}$, let us consider the function $\varphi=\sum_{g \in \Gamma_{N}} \mu(g) \times$ $\mathbb{1}_{g \Omega}$. Then

$$
\mu S_{T}(x, y)=\int \varphi(h) p_{T}^{\mu}(x h, y) d h, \quad x, y \in \Gamma_{N} .
$$

Since $\|\varphi\|_{1}=1$, it follows from (6.10) that

$$
\left\|\mu S_{T}-p_{T}^{\mu}\right\|_{1} \leq \frac{c}{\sqrt{T}}, \quad T \geq 1 .
$$

Since we also have

$$
\left\|p_{T+1}^{\mu}-p_{T}^{\mu}\right\|_{1} \leq \frac{c}{T}, \quad T \geq 1
$$

we conclude that

$$
\begin{aligned}
\left\|\left(\mu^{n-1}-p_{n-1}^{\mu}\right)\left(p_{1+T}^{\mu}-\mu S_{T}\right)\right\|_{\infty} & \leq\left\|\mu^{n-1}-p_{n-1}^{\mu}\right\|_{\infty}\left\|p_{1+T}^{\mu}-\mu S_{T}\right\|_{1} \\
& \leq c A \frac{1}{\sqrt{T}} n^{-(D+\gamma) / 2} .
\end{aligned}
$$

To estimate the remaining term in (11.8) we observe that

$$
\begin{aligned}
& \sum_{n / 2<i \leq n-2} p_{i}^{\mu}\left(p_{n-i+T}^{\mu}-\mu p_{n-i-1+T}^{\mu}\right)+p_{n-1}^{\mu}\left(p_{1+T}^{\mu}-\mu S_{T}\right) \\
& =p_{[n / 2]+1}^{\mu} p_{n-[n / 2]-1+T}^{\mu}-p_{n-1}^{\mu} \mu S_{T} \\
& \quad+\sum_{n / 2<i \leq n-2}\left(p_{i}^{\mu} \mu-p_{i+1}^{\mu}\right) p_{n-i-1+T}^{\mu}
\end{aligned}
$$

By (6.10) and (8.3),

$$
\begin{aligned}
\sum_{n / 2<i \leq n-2}\left\|p_{i}^{\mu} \mu-p_{i+1}^{\mu}\right\|_{\infty}\left\|p_{n-i-1+T}^{\mu}\right\|_{1} & \leq \sum_{n / 2<i \leq n-2} c i^{-(D+3) / 2} \\
& \leq c n^{-(D+1) / 2}
\end{aligned}
$$

Also, by (6.10), for all $x, y \in \Gamma_{N}$,

$$
\left|p_{n+T}^{\mu}(x, y)-\sum_{z \in \Gamma_{N}} p_{[n / 2]+1}^{\mu}(x, z) p_{n-[n / 2]-1+T}^{\mu}(z, y)\right| \leq c n^{-(D+1) / 2}
$$

and

$$
\left|p_{n+T}^{\mu}(x, y)-\sum_{z \in \Gamma_{N}} p_{n-1}^{\mu}(x, z)\left(\mu S_{T}\right)(z, y)\right| \leq c n^{-(D+1) / 2} .
$$

Hence

$$
\left\|p_{[n / 2]+1}^{\mu} p_{n-[n / 2]-1+T}^{\mu}-p_{n-1}^{\mu} \mu S_{T}\right\|_{\infty} \leq c n^{-(D+1) / 2} .
$$

The lemma follows by summing (11.9)-(11.14). 
12. Proof of the Taylor formula. In this section we give the proof of the Taylor formula (1.18) under the assumption that the Berry-Esseen estimate (11.2) holds.

We assume that $\Gamma_{N}=\Gamma$. The proof in the general case under a similar assumption (cf. Section 17) is exactly the same.

If $\Gamma$ is nilpotent and the torsion subgroup $\tau(\Gamma)$ is not trivial, then we can just extend the different kernels and functions from $\Gamma_{N}=\Gamma / \tau(\Gamma)$ to $\Gamma$, as explained in Section 1.4, and then the proofs remain exactly the same.

12.1. Polynomials on $\Gamma_{N}$. We use the notation of Sections 5 and 6.

Let us fix a monomial $P(t, x)=t^{i_{0}} x_{1}^{i_{1}} \cdots x_{q}^{i_{q}}$. Then, by Corollary 8.3,

$$
\left(\partial_{1}+(I-\mu)\right) P(t, x)=\left(\frac{\partial}{\partial t}+L_{\mu}\right) P(t, x)+Q(t, x),
$$

where $Q(t, x)$ is a polynomial satisfying

$$
\operatorname{deg}_{H} Q \leq \operatorname{deg}_{H} P-3 .
$$

On the other hand, it follows from (6.5) and (6.6) that, given any monomial $P(t, x)$ as above, there is a polynomial $W(t, x)$ such that

$$
\left(\frac{\partial}{\partial t}+L_{\mu}\right) W(t, x)=P(t, x)
$$

and

$$
\operatorname{deg}_{H} W(t, x)=\operatorname{deg}_{H} P(t, x)+2 .
$$

The following proposition is a consequence of the above observations.

Proposition 12.1. Given any monomial $P(t, x)$ on $N$, there is a polynomial $P^{\mu}(t, x)$ satisfying

$$
\begin{aligned}
\left(\frac{\partial}{\partial t}+L_{\mu}\right) P & =\left(\partial_{1}+(I-\mu)\right) P^{\mu}, \\
P^{\mu} & =P+W \\
\operatorname{deg}_{H} W & \leq \operatorname{deg}_{H} P-1 .
\end{aligned}
$$

Let the polynomials $P_{i}, i=0,1,2, \ldots$, be as in Section 6.1 and let us associate with those monomials and the sub-Laplacian $L_{\mu}$, polynomials $Q_{P_{i}}$, $i=0,1,2, \ldots$, satisfying (6.6). We associate with the polynomials $Q_{P_{i}}, i=$ $0,1,2, \ldots$, and fix, polynomials $Q_{P_{i}}^{\mu}, i=0,1,2, \ldots$, satisfying (12.3) above.

Note that for $0 \leq i \leq v_{2}$ we can take $Q_{P_{i}}^{\mu}=P_{i}$. Note also that $v_{0}=0, v_{1}=n_{1}$ and $n_{1} \leq n_{2}<v_{2}$. So we assume that $P_{0}(t, x)=Q_{P_{0}}^{\mu}(t, x)=1$, that $P_{i}(t, x)=$ $Q_{P_{i}}^{\mu}(t, x)=x_{i}$, for $1 \leq i \leq v_{2}$, and that $P_{i}=Q_{P_{i}}^{\mu}$, for $v_{1}<i \leq v_{2}$. 
12.2. A uniform approximation of a space-time $\mu$-harmonic function by an $L_{\mu}$-heat function. In this section we use the Berry-Esseen estimate (11.2) to prove the following lemma:

LEMMA 12.2. Let $V$ be as in Section 5.3 and let us assume that $\operatorname{supp} \mu \subseteq V$. Then there is a constant $c>0$ and $\beta \in(0,1)$ such that for all $\Theta_{1} \geq 4 \Theta_{2}, \Theta_{2} \geq 2, r \geq 1$ and all functions $u$ satisfying

$$
\left(\partial_{1}+(I-\mu)\right) u=0 \quad \text { in } \llbracket-\Theta_{1}^{2} r^{2}, \Theta_{1}^{2} r^{2} \rrbracket \times U^{\Theta_{1} r}
$$

we can associate a function $u^{\mu}$ satisfying

$$
\left(\frac{\partial}{\partial t}+L_{\mu}\right) u^{\mu}=0 \quad \text { in }\left(-\Theta_{2}^{2} r^{2}, \Theta_{2}^{2} r^{2}\right) \times V^{\Theta_{1} r}
$$

as well as $\left\|u^{\mu}\right\|_{\infty} \leq\|u\|_{\infty}$ and

$$
\sup \left\{\left|u-u^{\mu}\right| ; \llbracket-r^{2}, r^{2} \rrbracket \times U^{r}\right\} \leq c \Theta_{2}^{-\beta} r^{-\beta}+c e^{-\Theta_{1}^{2} / c \Theta_{2}^{2}} .
$$

As in Section 3.2, we use $Z_{n}$ to denote the right random walk with transition kernel $\mu(x, y)$ and by $\tau_{r}^{x}$ the stopping time

$$
\tau_{r}^{x}=\inf \left\{n: Z_{n} \notin x U^{r}\right\} .
$$

Using the Gaussian estimate (1.14), we can obtain the following improvement of Lemma 3.4.

LEMMA 12.3. There is a constant $c>0$ such that, for all $r, n \in \mathbb{N}$,

$$
P_{x}\left[\tau_{r}^{x} \leq n\right] \leq c \exp \left(-\frac{r^{2}}{c n}\right)
$$

PROOF. The proof follows the same lines as the proof of Lemma 3.4.

Let $a \geq 1$ such that $x U^{r} \cap y U^{r}=\varnothing$ when $y \notin x U^{a r}$. We have

$$
\begin{aligned}
\sum_{y \notin x U^{r}} \mu^{n}(x, y) & =P_{x}\left[Z_{n} \notin x U^{r}\right] \\
& \geq E^{P_{x}}\left[\mu^{n-\tau_{a r}^{x}}\left(Z_{\tau_{a r}^{x}}, \Gamma_{N} \backslash x U^{r}\right) ; \tau_{a r}^{x} \leq n\right] \\
& \geq E^{P_{x}}\left[\mu^{n-\tau_{a r}^{x}}\left(Z_{\tau_{a r}^{x}}, Z_{\tau_{a r}^{x}} U^{r}\right) ; \tau_{a r}^{x} \leq n\right] \\
& \geq E^{P_{x}}\left[\mu^{n-\tau_{a r}^{x}}\left(e, U^{r}\right) ; \tau_{a r}^{x} \leq n\right] .
\end{aligned}
$$

Now, we observe that (12.5) is interesting only for $r^{2} \geq n$ and that in that case, by (1.14), there is a $\delta>0$ such that

$$
\mu^{n-\tau_{a r}^{x}}\left(e, U^{r}\right) \geq \delta .
$$


So

$$
\sum_{y \notin x U^{r}} \mu^{n}(x, y) \geq \delta P_{x}\left[\tau_{a r}^{x} \leq r^{2}\right] .
$$

Since, by (1.14), there is a constant $c>0$ such that, for all $r, n \in \mathbb{N}$,

$$
\sum_{y \notin x U^{r}} \mu^{n}(x, y) \leq c \exp \left(-\frac{r^{2}}{c n}\right)
$$

we conclude that

$$
P_{x}\left[\tau_{a r}^{x} \leq n\right] \leq \frac{1}{\delta} c \exp \left(-\frac{r^{2}}{c n}\right),
$$

which proves the lemma.

LEMMA 12.4. Let $r, n \in \mathbb{N}$ and let $u$ be a function satisfying

$$
\left(\partial_{1}+(I-\mu)\right) u=0 \quad \text { in } \llbracket-2 n, 2 n \rrbracket \times x U^{2 r} .
$$

Then

$$
\left|u(n, x)-\sum_{y \in x U^{r}} \mu^{n}(x, y) u(0, y)\right| \leq 2\|u\|_{\infty} P_{x}\left[\tau_{r}^{x} \leq n\right] .
$$

Proof. We have

$$
\begin{aligned}
u(n, x)= & E^{P_{x}}\left[u\left(0, Z_{n}\right) ; \tau_{r}^{x}>n\right]+E^{P_{x}}\left[u\left(\tau_{r}^{x}, Z_{\tau_{r}^{x}}\right) ; \tau_{r}^{x} \leq n\right] \\
= & \sum_{y \in x U^{r}}\left(\mu^{n}(x, y)-E^{P_{x}}\left[\mu^{n-\tau_{r}^{x}}\left(Z_{\tau_{r}^{x}}, y\right) ; \tau_{r}^{x} \leq n\right]\right) u(0, y) \\
& +E^{P_{x}}\left[u\left(\tau_{r}^{x}, Z_{\tau_{r}^{x}}\right) ; \tau_{r}^{x} \leq n\right] .
\end{aligned}
$$

Hence

$$
\begin{aligned}
& \left|u(n, x)-\sum_{y \in x U^{r}} \mu^{n}(x, y) u(0, y)\right| \\
& \quad \leq\|u\|_{\infty}\left(E^{P_{x}}\left[\mu^{n-\tau_{r}^{x}}\left(Z_{\tau_{r}^{x}}, x U^{r}\right) ; \tau_{r}^{x} \leq n\right]+P_{x}\left[\tau_{r}^{x} \leq n\right]\right) \\
& \quad \leq 2\|u\|_{\infty} P_{x}\left[\tau_{r}^{x} \leq n\right]
\end{aligned}
$$

and the lemma follows.

Proof OF Lemma 12.2. Let $u$ satisfy

$$
\left(\partial_{1}+(I-\mu)\right) u=0 \quad \text { in } \llbracket-\Theta_{1}^{2} r^{2}, \Theta_{1}^{2} r^{2} \rrbracket \times x U^{\Theta_{1} r}
$$


and let us define, for $n>-\Theta_{2}^{2} r^{2}$ and $x \in \Gamma_{N}$,

$$
u_{1}(n, x)=\sum_{y \in U^{\Theta} r_{1} / 2} \mu^{n+\Theta_{2}^{2} r^{2}}(x, y) u\left(-\Theta_{2}^{2} r^{2}, y\right)
$$

and for $t>-\Theta_{2}^{2} r^{2}, x \in N$,

$$
u^{\mu}(t, x)=\sum_{y \in U^{\Theta} r^{r / 2}} p_{t+\Theta_{2}^{2} r^{2}}^{\varphi}(x, y) u\left(-\Theta_{2}^{2} r^{2}, y\right) .
$$

Now by (12.5) and (12.6),

$$
\begin{aligned}
\sup \left\{\left|u-u_{1}\right| ; \llbracket-\Theta_{2}^{2} r^{2}, \Theta_{2}^{2} r^{2} \rrbracket \times U^{\Theta_{2} r}\right\} & \leq 2\|u\|_{\infty} P_{x}\left[\tau_{\Theta_{1} r / 2}^{x} \leq \Theta_{2}^{2} r^{2}\right] \\
& \leq c\|u\|_{\infty} e^{-\Theta_{1}^{2} / c \Theta_{2}^{2}} .
\end{aligned}
$$

Also, by interpolating the Berry-Esseen estimate (11.2) and the Gaussian estimates (1.14) and (6.9), we have that there is a $\beta \in(0,1 / 2)$ such that

$$
\left\|\mu^{n}-p_{n}^{\mu}\right\|_{1} \leq c n^{-\beta}, \quad n \in \mathbb{N} .
$$

It follows that

$$
\sup \left\{\left|u_{1}-u^{\mu}\right| ;\left[-r^{2}, r^{2} \rrbracket \times U^{r}\right\} \leq c\|u\|_{\infty} \Theta_{2}^{-\beta} r^{-\beta} .\right.
$$

The lemma follows by summing (12.7) and (12.8).

12.3. The iteration argument. The following lemmas are inspired by Avellaneda and Lin $[9,10]$.

LEMMA 12.5. For all $n \in \mathbb{N}$ and $\eta \in(0,1)$ there are $r_{0}>1, \Theta>1$ and $c_{n}>0$ such that, for all $r \geq r_{0}$ and all functions $u$ satisfying

$$
\left(\partial_{1}+(I-\mu)\right) u=0 \quad \text { in } \llbracket-\Theta^{2} r^{2}, \Theta^{2} r^{2} \rrbracket \times U^{\Theta r},
$$

we have

$$
\sup \left\{\left|u-\sum_{0 \leq i \leq \nu_{n}} A_{i}(\Theta r)^{-\operatorname{deg}_{H} P_{i}} Q_{P_{i}}^{\mu}\right| ; \llbracket r^{2}, r^{2} \rrbracket \times U^{r}\right\}<\Theta^{-(n+\eta)}\|u\|_{\infty}
$$

where the constants $A_{i}$ satisfy

$$
\left|A_{i}\right| \leq c_{n}(\log \Theta)^{\operatorname{deg}_{H} P_{i}}\|u\|_{\infty},
$$

for all $0 \leq i \leq v_{n}$, and

$$
\left(\partial_{1}+(I-\mu)\right)\left(\sum_{v_{d-1} \leq i \leq v_{d}} A_{i} Q_{P_{i}}^{\mu}\right)=0
$$

for all $1 \leq d \leq n$. 
Proof. Let us fix $n \in \mathbb{N}, \mu \in(0,1), \Theta>16$ and a function $u$ satisfying (12.9).

Then, by Lemma 12.2 and by taking $\Theta_{1}=\Theta$ and $\Theta_{2}=\Theta / \log \Theta$, there is a function $u^{\mu}$ satisfying

$$
\left(\frac{\partial}{\partial t}+L_{\mu}\right) u^{\mu}=0 \quad \text { in }\left[-\left(\frac{\Theta}{\log \Theta}\right)^{2} r^{2},\left(\frac{\Theta}{\log \Theta}\right)^{2} r^{2}\right] \times V^{\Theta r / \log \Theta},
$$

as well as

$$
\left\|u^{\mu}\right\|_{\infty} \leq\|u\|_{\infty}
$$

and

$$
\begin{aligned}
& \sup \left\{\left|u-u^{\mu}\right| ; \llbracket\left[-r^{2}, r^{2} \rrbracket \times U^{r}\right\}\right. \\
& \quad \leq c\|u\|_{\infty}\left(\Theta^{-\beta}(\log \Theta)^{\beta} r^{-\beta}+e^{-(\log \Theta)^{2} / c}\right) .
\end{aligned}
$$

Also, by Theorem 6.2,

$$
\begin{aligned}
\sup \left\{\left|u^{\mu}-\sum_{0 \leq i \leq \nu_{n}} B_{i}\left(\frac{\Theta}{\log \Theta} r\right)^{-\operatorname{deg}_{H} P_{i}} Q_{P_{i}}\right| ;\left[-r^{2}, r^{2}\right] \times V^{r}\right\} \\
<c_{n}\left(\frac{\Theta}{\log \Theta}\right)^{-(n+1)}\left\|u^{\mu}\right\|_{\infty},
\end{aligned}
$$

where the constants $B_{i}$ satisfy

$$
\left|B_{i}\right| \leq c_{n}\left\|u^{\mu}\right\|_{\infty}
$$

for all $0 \leq i \leq v_{n}$, and

$$
\left(\frac{\partial}{\partial t}+L_{\mu}\right)\left(\sum_{v_{d-1} \leq i \leq v_{d}} B_{i} Q_{P_{i}}(t, x)\right)=0,
$$

for all $1 \leq d \leq n$.

Now let us observe that there is a constant $c>0$ such that

$$
\sup \left\{\left|Q_{P_{i}}-Q_{P_{i}}^{\mu}\right| ;\left[-r^{2}, r^{2}\right] \times V^{r}\right\} \leq c r^{\operatorname{deg}_{H} P_{i}-1}
$$

and hence

$$
\begin{aligned}
& \left(\frac{\Theta}{\log \Theta} r\right)^{-\operatorname{deg}_{H} P_{i}} \sup \left\{\left|Q_{P_{i}}-Q_{P_{i}}^{\mu}\right| ;\left[-r^{2}, r^{2}\right] \times V^{r}\right\} \\
& \quad \leq c\left(\frac{\Theta}{\log \Theta}\right)^{-\operatorname{deg}_{H} P_{i}} r^{-1} .
\end{aligned}
$$

Let us take

$$
A_{i}=B_{i}(\log \Theta)^{\operatorname{deg}_{H} P_{i}}, \quad 0 \leq i \leq v_{n} .
$$


Then, combining (12.11), (12.12) and (12.13), we have that

$$
\begin{aligned}
\sup \{\mid u & -\sum_{0 \leq i \leq \nu_{n}} A_{i}(\Theta r)^{-\operatorname{deg}_{H} P_{i}} Q_{P_{i}}^{\mu} \mid ; \llbracket\left[-r^{2}, r^{2} \rrbracket \times U^{r}\right\} \\
& \leq \sup \left\{\left|u^{\mu}-\sum_{0 \leq i \leq v_{n}} B_{i}(\log \Theta)^{\operatorname{deg}_{H} P_{i}}(\Theta r)^{-\operatorname{deg}_{H} P_{i}} Q_{P_{i}}\right| ; \llbracket\left[-r^{2}, r^{2} \rrbracket \times U^{r}\right\}\right. \\
& +\sup \left\{\left|u-u^{\mu}\right| ; \llbracket-r^{2}, r^{2} \rrbracket \times U^{r}\right\} \\
& +\sum_{0 \leq i \leq v_{n}}\left|B_{i}\right|(\log \Theta)^{\operatorname{deg}_{H} P_{i}}(\Theta r)^{-\operatorname{deg}_{H} P_{i}} \sup \left\{\left|Q_{P_{i}}-Q_{P_{i}}^{\mu}\right| ;\left[r^{2}, r^{2}\right] \times U^{r}\right\} \\
\leq & c_{n} \Theta{ }^{-(n+1)}(\log \Theta)^{n+1}\left\|u^{\mu}\right\|_{\infty}+c\|u\|_{\infty}\left((\log \Theta)^{\beta} \Theta^{-\beta} r^{-\beta}+e^{-(\log \Theta)^{2} / c}\right) \\
& +c(\log \Theta)^{n} \Theta^{-1} r^{-1} \sum_{0 \leq i \leq v_{n}}\left|B_{i}\right| \\
\leq & c_{n} \Theta{ }^{-(n+1)}(\log \Theta)^{n+1}\|u\|_{\infty}+c\|u\|_{\infty}\left((\log \Theta)^{\beta} \Theta^{-\beta} r^{-\beta}+e^{-(\log \Theta)^{2} / c}\right) \\
& +c(\log \Theta)^{n} \Theta^{-1} r^{-1} c_{n} v_{n}\|u\|_{\infty} .
\end{aligned}
$$

The lemma follows by taking $\Theta$ and $r_{0}$ large enough.

LEMMA 12.6. Let $\mu, \Theta$ and $r_{0}$ be as in the previous lemma. Then there is a constant $c_{n}>0$ such that, for all $m \in \mathbb{N}$, all $r \geq \Theta^{m-1} r_{0}$ and all functions $u$ satisfying

$$
\left(\partial_{1}+(I-\mu)\right) u=0 \quad \text { in } \llbracket-\Theta^{2 m} r^{2}, \Theta^{2 m} r^{2} \rrbracket \times U^{\Theta^{m} r},
$$

we have

$$
\begin{aligned}
\sup \left\{\left|u-\sum_{0 \leq i \leq \nu_{n}} A_{i}^{m}\left(\Theta^{m} r\right)^{-\operatorname{deg}_{H} P_{i}} Q_{P_{i}}^{\mu}\right| ; \llbracket-r^{2}, r^{2} \rrbracket \times U^{r}\right\} \\
<\Theta^{-m(n+\eta)}\|u\|_{\infty},
\end{aligned}
$$

where the constants $A_{i}^{m}$ satisfy

$$
\left|A_{i}^{m}\right| \leq c_{n}(\log \Theta)^{\operatorname{deg}_{H} P_{i}}\|u\|_{\infty},
$$

for all $0 \leq i \leq v_{n}$, and

$$
\left(\partial_{1}+(I-\mu)\right)\left(\sum_{v_{d-1}<i \leq v_{d}} A_{i}^{m} Q_{P_{i}}^{\mu}\right)=0,
$$

for all $1 \leq d \leq n$ 
ProOF. We prove the lemma by induction on $m$. For $m=1$ we are in the case of the previous lemma. So let us assume that (12.15) is true for some $m \in \mathbb{N}$. To prove that it is also true for $m+1$, let us assume, for simplicity, that $\|u\|_{\infty} \leq 1$. By the induction hypothesis

$\sup \left\{\left|u-\sum_{0 \leq i \leq \nu_{n}} A_{i}^{m}\left(\Theta^{m+1} r\right)^{-\operatorname{deg}_{H} P_{i}} Q_{P_{i}}^{\mu}\right| ; \llbracket\left[-\Theta^{2} r^{2}, \Theta^{2} r^{2} \rrbracket \times U^{\Theta r}\right\} \leq \Theta^{-m(n+\eta)}\right.$

We consider the function

$$
w=\Theta^{m(n+\eta)}=\left(u-\sum_{0 \leq i \leq \nu_{n}} A_{i}^{m}\left(\Theta^{m+1} r\right)^{-\operatorname{deg}_{H} P_{i}} Q_{P_{i}}^{\mu}\right) .
$$

Then

$$
\left(\partial_{1}+(I-\mu)\right) w=0 \quad \text { in } \llbracket-\Theta^{2} r^{2}, \Theta^{2} r^{2} \rrbracket \times U^{\Theta r}
$$

and

$$
\sup \left\{|w| ; \llbracket-\Theta^{2} r^{2}, \Theta^{2} r^{2} \rrbracket \times U^{\Theta r}\right\} \leq 1 .
$$

So, by Lemma 12.5 , we have that

$$
\sup \left\{\left|w-\sum_{0 \leq i \leq \nu_{n}} B_{i}(\Theta r)^{-\operatorname{deg}_{H} P_{i}} Q_{P_{i}}^{\mu}\right| ; \llbracket\left[-r^{2}, r^{2} \rrbracket \times U^{r}\right\}<\Theta^{-(n+\eta)},\right.
$$

where the constants $B_{i}$ satisfy

$$
\left|B_{i}\right| \leq c_{n}(\log \Theta)^{-\operatorname{deg}_{H} P_{i}}
$$

for all $0 \leq i \leq v_{n}$, and

$$
\left(\partial_{1}+(I-\mu)\right)\left(\sum_{v_{d-1}<i \leq v_{d}} B_{i} Q_{P_{i}}^{\mu}\right)=0
$$

for all $1 \leq d \leq n$.

So, if we set

$$
A_{i}^{m+1}=A_{i}^{m}+\Theta^{-m\left(n+\mu-\operatorname{deg}_{H} P_{i}\right)} B_{i}
$$

then we have

$$
\sup \left\{\left|u-\sum_{0 \leq i \leq v_{n}} A_{i}^{m+1}\left(\Theta^{m+1} r\right)^{-\operatorname{deg}_{H} P_{i}} Q_{P_{i}}^{\mu}\right| ; \llbracket-r^{2}, r^{2} \rrbracket \times U^{r}\right\}<\Theta^{-(m+1)(n+\eta)},
$$

which proves the inductive step and the lemma follows. 
12.4. The Taylor formula. The following result is the analogue of Theorem 1.11 when $\Gamma=\Gamma_{N}$.

THEOREM 12.7. For all $n \in \mathbb{N}$ there is a constant $c_{n}>0$ such that, for all $R \geq r \geq 1$ and all functions $u$ satisfying

$$
\left(\partial_{1}+(I-\mu)\right) u=0 \quad \text { in } \llbracket-R^{2}, R^{2} \rrbracket \times U^{R},
$$

we have

$$
\begin{aligned}
& \sup \left\{\left|u-\sum_{0 \leq i \leq v_{n}} A_{i} R^{-\operatorname{deg}_{H} P_{i}} Q_{P_{i}}^{\mu}\right| ; \llbracket-r^{2}, r^{2} \rrbracket \times U^{r}\right\} \\
& \quad \leq c_{n}\left(\frac{R}{r}\right)^{-(n+1)}\|u\|_{\infty},
\end{aligned}
$$

where the constants $A_{i}$ satisfy

$$
\left|A_{i}\right| \leq c_{n}\|u\|_{\infty}
$$

for all $0 \leq i \leq v_{n}$, and

$$
\left(\partial_{1}+(I-\mu)\right)\left(\sum_{v_{d-1}<i \leq v_{d}} A_{i} Q_{P_{i}}^{\mu}\right)=0,
$$

for all $1 \leq d \leq n$.

Proof. If $R \geq r \geq r_{0}$, then $\Theta^{m-1} r \leq R<\Theta^{m} r$ for some $m \in \mathbb{N}$ and hence (12.16) follows from Lemma 12.6.

If $R \geq r_{0}>r \geq 1$, then $R / r \leq r_{0} R / r_{0}$ and hence (12.16) follows in the same way from Lemma 12.6.

If $r_{0} \geq R \geq r \geq 1$, then (12.16) is trivial.

13. Harmonic functions of polynomial growth. In this section we give the proof of Theorem 1.12. We assume that $\Gamma_{N}=\Gamma$. The proof in the general case is exactly the same. If $\Gamma$ is nilpotent and we have set $\Gamma_{N}=\Gamma / \tau(\Gamma)$, then we can just extend the different polynomials from $\Gamma_{N}$ to $\Gamma$ (see Section 1.4) and then the proof below also works as is.

Proof OF THEOREM 1.12. Let $u$ be a $\mu$-harmonic function on $G$ which grows polynomially; that is, there are $c>0$ and $n \in \mathbb{N}$ such that

$$
\sup \left\{|u| ; U^{r}\right\} \leq c r^{n}, \quad r \geq 1 .
$$

Let the polynomials $Q_{P_{i}}^{\mu}(t, x)$ be as in section 12.1 and let us denote by $Q_{P_{i}}^{\mu}(x)$ their restrictions to $N$, that is, $Q_{P_{i}}^{\mu}(x)=Q_{P_{i}}^{\mu}(0, x), x \in N$. 
By (12.16), there is a $c>0$ such that, for all $r \geq k$

$$
\begin{aligned}
\sup \left\{\left|u-\sum_{0 \leq i \leq \nu_{n}} A_{i}^{r} r^{-\operatorname{deg}_{H} P_{i}} Q_{P_{i}}^{\mu}\right| ; U^{k}\right\} & <c\left(\frac{r}{k}\right)^{-(n+1)}\|u\|_{L^{\infty}\left(U^{r}\right)} \\
& \leq c k^{n+1} r^{-1},
\end{aligned}
$$

where the constants $A_{i}^{r}$ are such that

$$
(I-\mu)\left(\sum_{0 \leq i \leq \nu_{n}} A_{i}^{r} r^{-\operatorname{deg}_{H} P_{i}} Q_{P_{i}}^{\varphi}\right)=0 .
$$

For each $k \in \mathbb{N}$, let us choose $r_{k} \in \mathbb{N}$ such that

$$
c k^{n+1} r_{k}^{-1} \leq \frac{1}{k}
$$

We set $C_{k, i}=0$, for $Q_{P_{i}}^{\mu}=0$ and

$$
C_{k, i}=A_{i}^{r_{k}} r_{k}^{-\operatorname{deg}_{H} P_{i}}
$$

otherwise. Then (13.1) and (13.2) imply that there is a $c>0$ such that, for all $k \in \mathbb{N}$,

$$
\sup \left\{\left|u-\sum_{0 \leq i \leq \nu_{n}} C_{k, i} Q_{P_{i}}^{\mu}\right| ; U^{k}\right\}<\frac{1}{k}
$$

with

$$
(I-\mu)\left(\sum_{0 \leq i \leq \nu_{n}} C_{k, i} Q_{P_{i}}^{\mu}\right)=0 .
$$

Now, there are a subsequence $C_{k_{j}, i}$ and constants $C_{i}$ such that

$$
C_{k_{j}, i} \rightarrow C_{i} \quad \text { as } j \rightarrow \infty
$$

for all $0 \leq i \leq v_{n}$.

To see this, let us observe that if this were not the case, then we would have that

$$
M_{k}=\max \left\{\left|C_{k, i}\right|, 0 \leq i \leq v_{n}\right\} \rightarrow \infty \quad \text { as } k \rightarrow \infty .
$$

Since $\left|C^{k, i}\right| / M_{k} \leq 1$, there are a subsequence $C^{k_{\ell}, i}$ and constants $B_{i}$ such that

$$
\frac{C^{k_{\ell}, i}}{M_{k_{\ell}}} \rightarrow B_{i} \quad \text { as } k \rightarrow \infty
$$

for all $0 \leq i \leq v_{n}$. Note that the subsequence $C^{k_{\ell}, i}$ can be chosen is such a way that some of the constants $B_{i}$ are equal to 1 (and hence not all of them vanish). Let

$$
R(x)=\sum_{0 \leq i \leq \nu_{n}} B_{i} Q_{P_{i}}^{\varphi}(x)
$$


By (13.3)

$$
\frac{1}{M_{k_{\ell}}}\left(u(x)-\sum_{0 \leq i \leq \nu_{n}} C_{k_{\ell}, i} Q_{P_{i}}^{\mu}(x)\right) \rightarrow 0 \quad \text { as } k \rightarrow \infty
$$

for all $x \in \Gamma_{N}$. So, $R(x)=0$ for all $x \in \Gamma_{N}$.

Since

$$
\sup \left\{|R(x)| ; U^{r}\right\} \sim \sup \left\{\left|\sum_{v_{n-1}<i \leq v_{n}} B_{i} P_{i}(x)\right| ; U^{r}\right\} \quad \text { as } r \rightarrow \infty,
$$

we have that

$$
\sum_{v_{n-1}<i \leq \nu_{n}} B_{i} P_{i}(x)=0, \quad x \in \Gamma_{N}
$$

and hence $B_{i}=0, v_{n-1}<i \leq v_{n}$.

Arguing in the same way, we can prove successively that, for all $k=n-$ $1, \ldots, 1, B_{i}=0, v_{k-1}<i \leq v_{k}$, and that $B_{0}=0$. This is absurd because, by construction, not all of the coefficients $B_{i}$ vanish. We conclude therefore that (13.5) holds.

By letting $j \rightarrow \infty$, it follows from (13.3) and (13.4) that

$$
u(x)=\sum_{0 \leq i \leq \nu_{n}} C_{i} Q_{P_{i}}^{\mu}(x)
$$

for all $x \in \Gamma_{N}$ and the theorem follows.

14. The homogenized sub-Laplacian $L_{H \mu}$. The goal of this section is to define the homogenized operator $L_{H \mu}$ associated with the centered probability measure $\mu$ on $\Gamma$, when $\Gamma \neq \Gamma_{N} . L_{H \mu}$ is a centered left invariant sub-Laplacian on $N$ which, with the notation of Section 6, we write as

$$
L_{H \mu}=-\sum_{1 \leq i, j \leq n_{1}} q_{i j} X_{i} X_{j}-\sum_{n_{1}<i \leq n_{2}} q_{i} X_{i}
$$

The coefficients $q_{i j}$ and $q_{i}$ is are constant [and the $n_{1} \times n_{1}$ matrix $\left(q_{i j}\right)$ is symmetric and positive definite].

The way $L_{H \mu}$ and $\mu$ are related is illustrated by (14.9) below.

14.1. The coefficients $a_{i j}$ and $a_{i}$. Let $f \in C^{\infty}(N)$ and let us extend $f$ to $\Gamma$ by setting $f\left(x g_{i}\right)=f(x), x \in \Gamma_{N}, 0 \leq i \leq k$.

Let the monomials $P_{i}$ be as in Section 6.1. We extend these monomials to $\Gamma$ by setting $P_{i}\left(t, x g_{i}\right)=P_{i}(t, x), x \in N, 0 \leq i \leq k$. We set $P_{i}(x)=P_{i}(0, x)$. 
Let

$$
\begin{aligned}
b_{i}\left(g_{\ell}\right) & =\sum_{h \in \Gamma} P_{i}\left(g_{\ell} h\right) \mu(h), \quad 1 \leq i \leq n_{2}, \\
a_{i j}\left(g_{\ell}\right) & =\sum_{h \in \Gamma} P_{i}\left(g_{\ell} h\right) P_{j}\left(g_{\ell} h\right) \mu(h), \quad 1 \leq i, j \leq n_{1}, \\
a_{i}\left(g_{\ell}\right) & =b_{i}\left(g_{\ell}\right), \quad 1 \leq i \leq n_{1}, \\
a_{i}\left(g_{\ell}\right) & =b_{i}\left(g_{\ell}\right)-\frac{1}{2} \sum_{1 \leq \lambda<\mu \leq n_{1}} a_{\lambda \mu}\left(g_{\ell}\right) \operatorname{pr}_{i}\left[X_{\lambda}, X_{\mu}\right]_{N}, \quad n_{1}<i \leq n_{2} .
\end{aligned}
$$

Note that, by setting $a_{i}\left(x g_{\ell}\right)=a_{i}\left(g_{\ell}\right)$ and $a_{i j}\left(x g_{\ell}\right)=a_{i j}\left(g_{\ell}\right), x \in \Gamma_{N}$, these coefficients become functions of type P (cf. Section 1.4).

LEMMA 14.1. We have

$$
\sum_{0 \leq \ell \leq k} a_{i}\left(g_{\ell}\right)=0, \quad 1 \leq i \leq n_{1} .
$$

PROOF. If $g=x g_{\ell}$ with $x \in \Gamma_{N}$ and $0 \leq \ell \leq k$, then, using the notation of Section 1.3, we set $g_{N}=x$ and $\bar{g}=g_{\ell}$. Let

$$
\zeta_{i}(g)=\sum_{\bar{w}} P_{i}(\bar{w} g), \quad 1 \leq i \leq n_{1} .
$$

Clearly, to prove (14.2) it is enough to prove that

$$
\sum_{g \in \Gamma} \zeta_{i}(g) \mu(g)=0, \quad 1 \leq i \leq n_{1} .
$$

We have

$$
\begin{aligned}
\zeta_{i}(g h) & =\sum_{\bar{w}} P_{i}(\bar{w} g h)=\sum_{\bar{w}} P_{i}\left((\bar{w} g)_{N} \overline{\bar{w} g} h\right) \\
& =\sum_{\bar{w}} P_{i}\left((\bar{w} g)_{N}\right)+\sum_{\bar{w}} P_{i}(\overline{\bar{w} g} h)=\sum_{\bar{w}} P_{i}(\bar{w} g)+\sum_{\bar{w}} P_{i}(\bar{w} h) \\
& =\zeta_{i}(g)+\zeta_{i}(h) .
\end{aligned}
$$

This shows that the functions $\zeta_{i}, 1 \leq i \leq n_{1}$, are additive, and hence (14.3) follows from the definition of a centered probability measure.

Let $V$ be as in Section 6.5 and let us also assume that $\left(g_{\ell} h\right)_{N} \in V$ for all $h \in \operatorname{supp} \mu$ and $0 \leq i \leq k$.

The following lemma is a consequence of Lemma 5.3 and (14.1) and (14.2) above.

LeMma 14.2. Let $f$ be as above. Also, let $\psi$ be a function of type P. Then there is a $c>0$ independent of $f$ such that, for all $x \in \Gamma_{N}$ and $0 \leq \ell \leq k$, the following hold: 
(i)

$$
\begin{aligned}
(I-\mu) f\left(x g_{\ell}\right)= & -\sum_{1 \leq i \leq n_{1}} a_{i}\left(g_{\ell}\right) X_{i} f(x)-\frac{1}{2} \sum_{1 \leq i, j \leq n_{1}} a_{i j}\left(g_{\ell}\right) X_{i} X_{j} f(x) \\
& -\sum_{n_{1}<i \leq n_{2}} a_{i}\left(g_{\ell}\right) X_{i} f(x)+F\left(x g_{\ell}\right)
\end{aligned}
$$

with

$$
\left|F\left(x g_{\ell}\right)\right| \leq c\left\|\nabla_{X}^{3} f\right\|_{L^{\infty}(x V)}
$$

(ii) for all $1 \leq v \leq n_{1}$,

$$
\begin{aligned}
& (I-\mu)\left(\varphi X_{v} f\right)\left(x g_{\ell}\right) \\
& \quad=((I-\mu) \psi)\left(g_{\ell}\right) X_{v} f(x)-\sum_{1 \leq i \leq n_{1}} \mu\left(\psi P_{i}\right)\left(g_{\ell}\right) X_{i} X_{n} f(x)+F\left(x g_{\ell}\right)
\end{aligned}
$$

with

$$
\left|F\left(x g_{\ell}\right)\right| \leq c\left\|\nabla_{X}^{3} f\right\|_{L^{\infty}(x V)}
$$

14.2. The correctors and the homogenized operator $L_{H \varphi}$. The definition of the correctors $\psi^{j}, j=1, \ldots, n_{1}$, is motivated by (14.4).

DEFINITION. We define the (first order) correctors $\psi^{j}, 1 \leq j \leq n_{1}$ (cf. [13, 26]), as functions of type $P$ satisfying

$$
(I-\mu) \psi^{j}=a_{j} \quad \text { and } \quad\left\langle\psi^{j}\right\rangle=0 .
$$

Note that the correctors $\psi^{j}$ are well defined and they are given by $\psi^{j}=$ $\sum_{n \geq 0} \mu^{n} a_{j}, 1 \leq j \leq n_{1}$ (cf. Section 1.4).

Let

$$
b_{i j}\left(g_{\ell}\right)=\sum_{h \in \Gamma} \psi^{j}\left(g_{\ell} h\right) P_{i}\left(g_{\ell} h\right) \mu(h), \quad 1 \leq i, j \leq n_{1} .
$$

If $f$ is as in Lemma 14.2, then combining (14.4), (14.5) and (14.6) we have that, for all $x \in \Gamma_{N}$ and $0 \leq \ell \leq k$,

$$
\begin{aligned}
(I-\mu) & \left(f+\sum_{1 \leq j \leq n_{1}} \psi^{j} X_{j} f\right)\left(x g_{\ell}\right) \\
= & -\sum_{1 \leq i, j \leq n_{1}}\left(\frac{1}{2} a_{i j}\left(g_{\ell}\right)+b_{i j}\left(g_{\ell}\right)\right) X_{i} X_{j} f(x) \\
& -\sum_{n_{1}<i \leq n_{2}} a_{i}\left(g_{\ell}\right) X_{i} f(x)+F\left(x g_{\ell}\right)
\end{aligned}
$$


with

$$
\left|F\left(x g_{\ell}\right)\right| \leq c\left\|\nabla_{X}^{3} f\right\|_{L^{\infty}(x V)} .
$$

The following definitions are motivated by the expression (14.7) above.

DEFINITION. The homogenized sub-Laplacian $L_{H \mu}$ associated with $\mu$ is defined with be the operator

$$
L_{H \mu}=-\sum_{1 \leq i, j \leq n_{1}} q_{i j} X_{i} X_{j}-\sum_{n_{1}<i \leq n_{2}} q_{i} X_{i}
$$

with coefficients defined by

$$
\begin{aligned}
q_{i j} & =\left\langle\frac{1}{2} a_{i j}+b_{i j}\right\rangle, \quad 1 \leq i, j \leq n_{1}, \\
q_{i} & =\left\langle b_{i}\right\rangle, \quad n_{1}<i \leq n_{2} .
\end{aligned}
$$

Definition. We define the (second order) correctors $\psi^{i j}, 1 \leq i, j \leq n_{1}$ (cf. $[13,26])$, as functions of type $P$ satisfying

$$
(I-\mu) \psi^{i j}=\frac{1}{2} a_{i j}+b_{i j}-q_{i j}, \quad\left\langle\psi^{i j}\right\rangle=0 .
$$

We also define the (second order) correctors $\psi^{j}, n_{1}<j \leq n_{2}$, as continuous functions on $M$ satisfying

$$
(I-\mu) \psi^{j}=a_{j}-q_{j}, \quad\left\langle\psi^{j}\right\rangle=0 .
$$

The following lemma is a direct consequence of (14.7) and the above definitions.

LEMMA 14.3. There is a $c>0$ such that, for all functions $f$ as in Lemma 14.2 and all $x \in \Gamma_{N}$ and $0 \leq \ell \leq n_{1}$,

$$
\left|(I-\mu)\left(f\left(x g_{\ell}\right)+\sum_{1 \leq j \leq n_{2}} \psi^{j}\left(g_{\ell}\right) X_{j} f(x)\right)\right| \leq c\left\|\nabla_{X}^{2} f\right\|_{L^{\infty}(x V)},
$$

$$
(I-\mu)\left(f+\sum_{1 \leq j \leq n_{2}} \psi^{j} X_{j} f+\sum_{1 \leq i, j \leq n_{1}} \psi^{i j} X_{j} f\right)=L_{H \varphi} f+F
$$

with the function $F$ satisfying

$$
\left|F\left(x g_{\ell}\right)\right| \leq c\left\|\nabla_{X}^{3} f(x)\right\|_{L^{\infty}(x V)} .
$$


Corollary 14.4. Let $u \in C^{\infty}(\mathbb{R} \times N)$ and let us extend $u$ to $\mathbb{R} \times \Gamma$ by setting $u\left(t, x g_{\ell}\right)=u(t, x), x \in \Gamma_{N}, 0 \leq \ell \leq n_{1}$. Also, let

$$
\begin{aligned}
U\left(t, x g_{\ell}\right)= & u\left(t, x g_{\ell}\right)+\sum_{1 \leq j \leq n_{2}} \psi^{j}\left(g_{\ell}\right) X_{j} u\left(t, x g_{\ell}\right) \\
& +\sum_{1 \leq i, j \leq n_{1}} \psi^{i j}\left(g_{\ell}\right) X_{i} X_{j} u\left(t, x g_{\ell}\right) .
\end{aligned}
$$

Then there is a constant $c>0$ independent of $u$ such that, for all $t \in \mathbb{R}, x \in N$ and $0 \leq \ell \leq k$,

$$
U\left(t+1, x g_{\ell}\right)-\mu U\left(t, x g_{\ell}\right)=\left(\frac{\partial}{\partial t}+L_{H \mu}\right) U(t, x)+V\left(t, x g_{\ell}\right)
$$

with

$$
\left|V\left(t, x g_{\ell}\right)\right| \leq c_{1}\left\|\left|\frac{\partial^{2}}{\partial s^{2}} u(s, x)\right|+\left|\nabla_{X} \frac{\partial}{\partial s} u(s, x)\right|+\left|\nabla_{X}^{3} u(t, x)\right|\right\|_{L^{\infty}([t, t+1] \times x V)} .
$$

14.3. $L_{H \mu}$ is a centered sub-Laplacian on $N$. The following lemma asserts that $L_{H \mu}$ is indeed a sub-Laplacian on $N$.

LEMmA 14.5. For all $\xi=\left(\xi_{1}, \ldots, \xi_{n_{1}}\right) \in \mathbb{R}^{n_{1}}, \xi \neq 0$,

$$
\sum_{1 \leq i, j \leq n_{1}} q_{i j} \xi_{i} \xi_{j}>0
$$

Proof. Let us fix $\xi=\left(\xi_{1}, \ldots, \xi_{n_{1}}\right) \neq 0$ and consider the function

$$
u=\sum_{1 \leq i \leq n_{1}} \xi_{i}\left(P_{i}+\psi^{i}\right) \text {. }
$$

Since by construction $(I-\mu)\left(P_{i}+\psi^{i}\right)=0,1 \leq i \leq n_{1}$, we have that $(I-\mu)$ $\times u=0$; that is, $u$ is $\mu$-harmonic. Since the function $f(t)=t^{2}$ is convex, we have that $(I-\mu) u^{2} \leq 0$.

We have

$$
\begin{aligned}
u^{2} & =\sum_{1 \leq i, j \leq n_{1}} \xi_{i} \xi_{j}\left(P_{i}+\psi^{i}\right)\left(P_{j}+\psi^{j}\right) \\
& =\sum_{1 \leq i, j \leq n_{1}} \xi_{i} \xi_{j}\left(P_{i} P_{j}+P_{i} \psi^{j}+P_{j} \psi^{i}+\psi^{i} \psi^{j}\right) .
\end{aligned}
$$

By Lemma 14.2, for $1 \leq i, j \leq n_{1}$,

$$
\begin{aligned}
& (I-\mu)\left(P_{i} P_{j}\right)=-P_{i} a_{j}-P_{j} a_{i}-a_{i j}, \\
& (I-\mu)\left(P_{i} \psi^{j}\right)=P_{i} a_{j}-b_{i j}, \\
& (I-\mu)\left(P_{j} \psi^{i}\right)=P_{j} a_{i}-b_{j i} .
\end{aligned}
$$


Hence

$$
(I-\mu)\left[\left(P_{i}+\psi^{i}\right)\left(P_{j}+\psi^{j}\right)\right]=-a_{i j}-b_{i j}-b_{j i}+\left[(I-\mu)\left(\psi^{i} \psi^{j}\right)\right] .
$$

It follows that $(I-\mu) u^{2}$ is a function of type P. Since $\left\langle(I-\mu)\left(\psi^{i} \psi^{j}\right)\right\rangle=0$, we have

$$
\left\langle(I-\mu)\left(\left(P_{i}+\psi^{i}\right)\left(P_{j}+\psi^{j}\right)\right)\right\rangle=-2 q_{i j}
$$

Hence

$$
\left\langle(I-\mu) u^{2}\right\rangle=-2 \sum_{1 \leq i, j \leq n_{1}} q_{i j} \xi^{i} \xi^{j} .
$$

Now, if we had $\sum_{1 \leq i, j \leq n_{1}} q_{i j} \xi_{i} \xi_{j}=0$, then we would have $(I-\mu) u^{2}=0$. Since the function $f(t)=t^{2}$ is strictly convex, this would imply that $u=$ const, which is absurd.

15. Proof of Propositions 1.3 and 1.4 in the general case. The proof of Propositions 1.3 and 1.4 in the general case is similar to the proof in the case $\Gamma=\Gamma_{N}$ (see Section 9). The only difference is that instead of Lemma 9.1 we must use the following generalization:

LEMMA 15.1. There is a constant $c>0$ such that, for all $n \in \mathbb{N}, T \geq 1$,

$$
\left\|p_{n+T}^{H \mu}-\mu^{n} p_{T}^{H \varphi}\right\|_{\infty} \leq c T^{-(D+1) / 2} .
$$

PROOF. Let

$$
U_{t}=p_{t}^{H \mu}-\sum_{1 \leq j \leq n_{2}} \psi^{j} X_{j} p_{t}^{H \mu}-\sum_{1 \leq i, j \leq n_{1}} \psi^{i j} X_{i} X_{j} p_{t}^{H \mu} .
$$

By (6.10), there is a $c>0$ such that, for all $T \geq 1$ and $t \geq 0$,

$$
\left\|X_{j} p_{t+T}^{H \mu}\right\|_{\infty} \leq c(t+T)^{-(D+1) / 2}, \quad\left\|X_{i} X_{j} p_{t+T}^{H \mu}\right\|_{\infty} \leq c(t+T)^{-(D+2) / 2} .
$$

So to prove (15.1) it is enough to prove that there is a $c>0$ such that, for all $T \geq 1$ and $t \geq 0$,

$$
\left\|U_{n+T}-\mu^{n} U_{T}\right\|_{\infty} \leq c T^{-(D+1) / 2} .
$$

By (14.10) and (6.10) there is a $c>0$ such that, for all $t \geq 1$,

$$
\left\|U_{t+1}-\mu U_{t}\right\|_{\infty} \leq c t^{-(D+3) / 2} \text {. }
$$

We have

$$
\begin{aligned}
U_{n+T}-\mu^{n} U_{T} & =U_{n+T}-\mu^{n-1} U_{1+T}+\mu^{n-1} U_{1+T}-\mu^{n} U_{T} \\
& =\sum_{0 \leq i \leq n-1} \mu^{i}\left(U_{n-i+T}-\mu U_{n-i-1+T}\right) .
\end{aligned}
$$


So, by (15.3),

$$
\begin{aligned}
\left\|U_{n+T}-\mu^{n} U_{T}\right\|_{\infty} & \leq \sum_{0 \leq i \leq n-1}\left\|\mu^{i}\right\|_{1}\left\|U_{n-i+T}-\mu U_{n-i-1+T}\right\|_{\infty} \\
& \leq c \sum_{0 \leq i \leq n-1}(n-i-1+T)^{-(D+3) / 2} \\
& \leq c T^{-(D+1) / 2}
\end{aligned}
$$

and the lemma follows.

16. Proof of the Gaussian estimate in the general case. The of proof of (1.14) follows the same lines as in the case $\Gamma_{N}=\Gamma$ (see Section 10). The only change is that we must replace the functions $H_{k}$ by their modifications $U_{k}, k \geq 1$.

16.1. The functions $U_{k}$. Let the family of functions $\rho_{k}$ be as in Section 10.1, let us fix $A>0$ and $B>0$ and let

$$
H_{k}(t, x)=\exp \left(-\frac{\left(\rho_{k}(x)+B \sqrt{k}\right)^{2}}{A(k+t)}\right), \quad t \geq 0, x \in N .
$$

We extend the functions $\rho_{k}$ and $H_{k}$ to $\Gamma$ by setting $\rho_{k}\left(x g_{\ell}\right)=\rho_{k}(x)$ and $H_{k}\left(t, x g_{\ell}\right)=H_{k}(t, x), x \in \Gamma_{N}, 0 \leq \ell \leq k$.

We do the same for the derivatives $X Y \cdots Z H_{k}, X, Y, \ldots, Z \in \mathfrak{n}$.

We consider the functions

$$
U_{k}=H_{k}(t, x)+\sum_{1 \leq j \leq n_{1}} \psi^{j} X_{j} H_{k}
$$

LEMMA 16.1. There are constants $A>0$ and $B>0$ such that, for all $k \geq 1$, $t \in[0, k]$ and all $|x|_{\Gamma} \leq a k$,

$$
0<\frac{1}{2} H_{k}(t, x)<U_{k}(t, x)<2 H_{k}(t, x)
$$

and

$$
U_{k}(t+1, x)>\mu U_{k}(t, x) .
$$

ProOF. For all $X \in \mathfrak{n}$, we have

$$
X H_{k}(t, x)=-\frac{1}{A} \frac{1}{k+t} 2\left(\rho_{k}(x)+B \sqrt{k}\right) X \rho_{k}(x) H_{k}(t, x) .
$$

It follows that there is a $c>0$ such that, for all $|x|_{\Gamma} \leq a k$,

$$
\left|X H_{k}(t, x)\right| \leq c \frac{1}{A}\left(a+\frac{B}{\sqrt{k}}\right) H_{k}(t, x),
$$

which proves (16.1). 
The proof of (16.2) is similar to the proof of (10.6) in the case $\Gamma=\Gamma_{N}$. We observe again that

$$
U_{k}(t+1, x)-\mu U_{k}(t, x)=U_{k}(t+1, x)-U_{k}(t, x)+(I-\mu) U_{k}(t, x) .
$$

Since the correctors $\psi^{j}$ are functions defined on $\Gamma / \Gamma_{N}$, which is finite, there is a $c>0$ such that

$$
\begin{aligned}
U_{k}(t+1, x)-U_{k}(t, x) \geq & \inf \left\{\frac{\partial}{\partial s} H_{k}(s, x) ; s \in[t, t+1]\right\} \\
& -c \sup \left\{\left|\nabla_{X} \frac{\partial}{\partial s} H_{k}(s, y)\right| ; s \in[t, t+1], y \in x V\right\} .
\end{aligned}
$$

Also, by (14.8),

$$
\left|(I-\mu) U_{k}(t, x)\right| \leq c \sup \left\{\nabla_{X}^{2} H_{k}(t, y) ; y \in x V\right\} .
$$

It follows that

$$
\begin{aligned}
U_{k}(t+ & 1, x)-\mu U_{k}(t, x) \\
\geq & \inf \left\{\frac{\partial}{\partial s} H_{k}(s, x) ; s \in[t, t+1]\right\} \\
& -c \sup \left\{\left|\nabla_{X} \frac{\partial}{\partial s} H_{k}(s, y)\right| ; s \in[t, t+1], y \in x V\right\} \\
& -c \sup \left\{\nabla_{X}^{2} H_{k}(t, y) ; y \in x V\right\} .
\end{aligned}
$$

As in the proof of (10.6) we observe that

$$
\frac{\partial}{\partial t} H_{k}(t, x)=\frac{1}{A} \frac{1}{k+t} \frac{\left(\rho_{k}(x)+B \sqrt{k}\right)^{2}}{k+t} H_{k}(t, x)
$$

and that, for all $X, Y \in \mathfrak{n}$,

$$
\begin{aligned}
\frac{\partial}{\partial t} X H_{k}(t, x)=\frac{1}{A} \frac{1}{k+t}[ & {\left[2 \frac{\rho_{k}(x)+B \sqrt{k}}{k+t} X \rho_{k}(x)\right.} \\
& -\frac{\left(\rho_{k}(x)+B \sqrt{k}\right)^{2}}{k+t} \frac{1}{A} \frac{1}{k+t} 2\left(\rho_{k}(x)\right. \\
& \left.+B \sqrt{k}) X \rho_{k}(x)\right] H_{k}(t, x) \\
=\frac{1}{A} \frac{1}{k+t}[ & {\left[2 \frac{\rho_{k}(x)+B \sqrt{k}}{k+t} X \rho_{k}(x)\right.} \\
& \left.-2 \frac{1}{A} \frac{1}{(k+t)^{2}}\left(\rho_{k}(x)+B \sqrt{k}\right)^{3} X \rho_{k}(x)\right] H_{k}(t, x)
\end{aligned}
$$


and

$$
\begin{aligned}
& X Y H_{k}(t, x) \\
&=-\frac{1}{A} \frac{1}{k+t}[ 2 X \rho_{k}(x) Y \rho_{k}(x)-2\left(\rho_{k}(x)+B \sqrt{k}\right) X Y \rho_{k}(x) \\
&\left.+\frac{1}{A} \frac{1}{(k+t)} 4\left(\rho_{k}(x)+B \sqrt{k}\right)^{2} X \rho_{k}(x) Y \rho_{k}(x)\right] H_{k}(t, x) .
\end{aligned}
$$

Case I $\left(|x|_{\Gamma} \leq \sqrt{k}-1\right.$ and $\left.t \in[0, k]\right)$. By construction, for all $|x|_{\Gamma} \leq \sqrt{k}$,

$$
U_{k}(t, x)=H_{k}(t, x)=\exp \left(-\frac{B^{2} k}{A(k+t)}\right) .
$$

Hence, for all $|x|_{\Gamma} \leq \sqrt{k}-1$,

$$
U_{k}(t+1, x)>U_{k}(t, x)=\left(\mu U_{k+1}(t, \cdot)\right)(x) .
$$

Case II $\left(\sqrt{k}-1 \leq|x|_{\Gamma} \leq \zeta \sqrt{k}+1\right.$ and $\left.0 \leq t \leq k\right)$. Then by (16.5)-(16.7) there is a $c>0$ such that

$$
\begin{aligned}
& U_{k}(t+1, x)-\mu U_{k}(t, x) \\
& \begin{aligned}
& \geq \frac{1}{A} \frac{1}{k+t+1}[ \frac{B^{2} k}{k+k+1}-c \frac{c \sqrt{k}+B \sqrt{k}}{k} \\
&-c \frac{1}{A} \frac{1}{k^{2}}(c \sqrt{k}+B \sqrt{k})^{3}-c-c(c \sqrt{k}+B \sqrt{k}) \frac{1}{\sqrt{k}} \\
&\left.-c \frac{1}{A} \frac{1}{k}(c \sqrt{k}+B \sqrt{k})^{2}-c(c \sqrt{k}+B \sqrt{k}) \frac{1}{\sqrt{k}}\right] H_{k}(t, x) \\
& \geq \frac{1}{A} \frac{1}{k+t+1}\left[\frac{B^{2}}{3}-c \frac{c+B}{\sqrt{k}}-c \frac{1}{A} \frac{1}{\sqrt{k}}(c+B)^{3}\right. \\
&\left.\quad-c-c(c+B)-c \frac{1}{A}(c+B)^{2}-c(c+B)\right] H_{k}(t, x) .
\end{aligned}
\end{aligned}
$$

So, by choosing $B$ large enough that

$$
\frac{B^{2}}{6}>+c(c+B)+c+c(c+B)+c(c+B)
$$

and then $A$ large enough that

$$
\frac{B^{2}}{6}>c \frac{1}{A}(c+B)^{3}+c \frac{1}{A}(c+B)^{2},
$$

we have

$$
U_{k}(t+1, x)>\mu U_{k}(t, x)
$$


Case III $\left(\zeta \sqrt{k}+1<|x|_{\Gamma}<a k\right.$ and $\left.0 \leq t \leq k\right)$. Then by (16.5)-(16.7) there is a $c>0$ such that

$$
\begin{aligned}
& U_{k}(t+1, x)-\mu U_{k}(t, x) \\
& \geq \frac{1}{A} \frac{1}{k+t+1}\left[\frac{\left(|x|_{\Gamma}+B \sqrt{k}\right)^{2}}{k+t+1}-c \frac{c|x|_{\Gamma}+B \sqrt{k}}{k+t}\right. \\
& -c \frac{1}{A} \frac{1}{k^{2}}\left(|x|_{\Gamma}+B \sqrt{k}\right)^{3}-c-c\left(|x|_{\Gamma}+B \sqrt{k}\right) \frac{1}{|x|_{\Gamma}} \\
& \left.-c \frac{1}{A} \frac{1}{k}\left(|x|_{\Gamma}+B \sqrt{k}\right)^{2}-c\left(|x|_{\Gamma}+B \sqrt{k}\right) \frac{1}{|x|_{\Gamma}}\right] H_{k}(t, x) \\
& \geq \frac{1}{A} \frac{1}{k+t+1}\left[\left(|x|_{\Gamma}+B \sqrt{k}\right) \frac{|x|_{\Gamma}+B \sqrt{k}}{3 k}-c \frac{|x|_{\Gamma}+B \sqrt{k}}{k}\right. \\
& -c \frac{1}{A}\left(\frac{|x|_{\Gamma}+B \sqrt{k}}{k}\right)^{2}\left(|x|_{\Gamma}+B \sqrt{k}\right)-c-c\left(1+\frac{B \sqrt{k}}{|x|_{\Gamma}}\right) \\
& \left.-c \frac{1}{A} \frac{|x|_{\Gamma}+B \sqrt{k}}{k}\left(|x|_{\Gamma}+B \sqrt{k}\right)-c\left(1+\frac{B \sqrt{k}}{|x|_{\Gamma}}\right)\right] H_{k}(t, x) .
\end{aligned}
$$

So, by choosing $B$ large enough that

$$
\left(|x|_{\Gamma}+B \sqrt{k}\right) \frac{|x|_{\Gamma}+B \sqrt{k}}{6 k}>c \frac{|x|_{\Gamma}+B \sqrt{k}}{k}+c+c\left(1+\frac{B \sqrt{k}}{|x|_{\Gamma}}\right)+c\left(1+\frac{B \sqrt{k}}{|x|_{\Gamma}}\right)
$$

and $A$ large enough that

$$
\begin{aligned}
\left(|x|_{\Gamma}+B \sqrt{k}\right) \frac{|x|_{\Gamma}+B \sqrt{k}}{6 k}> & \frac{1}{A}\left(\frac{|x|_{\Gamma}+B \sqrt{k}}{k}\right)^{2}\left(|x|_{\Gamma}+B \sqrt{k}\right) \\
& +c \frac{1}{A} \frac{|x|_{\Gamma}+B \sqrt{k}}{k}\left(|x|_{\Gamma}+B \sqrt{k}\right)
\end{aligned}
$$

we have

$$
U_{k}(t+1, x)>\mu U_{k}(t, x),
$$

which ends the proof of the lemma.

17. The proof of the Berry-Esseen estimate in the general case. In this section, we give the proof of Theorem 11.1 in the case when $\Gamma \neq \Gamma_{N}$. The general strategy is the same.

Let $L_{H \mu}$ be the homogenized sub-Laplacian associated with $\mu$ and let $p_{t}^{H \mu}(x, y)$ be its heat kernel. We extend $p_{t}^{H \mu}(x, y)$ to $\Gamma$ by setting

$$
p_{t}^{H \varphi}\left(x g_{i}, y g_{j}\right)=\frac{1}{k+1} p_{t}^{H \varphi}(x, y), \quad x, y \in N, 0 \leq i, j \leq k .
$$


Let us also recall that by (4.2) there are $\gamma \in(0,1]$ and $c>0$ such that

$$
\left\|\nabla_{U} \mu^{n}\right\|_{\infty} \leq c n^{-(D+\gamma) / 2}, \quad n \in \mathbb{N} .
$$

The following result is a generalization of Theorem 11.1.

THEOREM 17.1. There is a $c>1$ such that, for all $x, y \in \Gamma$ and $n \in \mathbb{N}$,

$$
\left|\mu^{n}(x, y)-p_{n}^{H \mu}(x, y)\right| \leq c n^{-(D+\gamma) / 2} \text {. }
$$

We point out again that, once we have proved Theorem 1.14, the above inequality will hold with $\gamma=1$.

Let the kernel $S_{t}(x, y)$ be as in Section 11 and let us extend it to $\Gamma$ by setting

$$
S_{t}\left(x g_{i}, y g_{j}\right)=\frac{1}{k+1} S_{t}(x, y), \quad x, y \in N, 0 \leq i, j \leq k .
$$

Theorem 17.1 is proved in exactly the same way as Theorem (11.1), once we have the following analogues of Lemmas 11.2 and 11.3.

LEMMA 17.2. There are constants $a, b \geq 1$ such that, for all $T \geq 1$ and $n \in \mathbb{N}$,

$$
\left\|\mu^{n}-p_{n}^{H \mu}\right\|_{\infty} \leq a\left\|\left(\mu^{n}-p_{n}^{H \mu}\right) S_{T}\right\|_{\infty}+b \sqrt{T} n^{-(D+\gamma) / 2} .
$$

The proof of the above lemma is exactly the same as the proof of Lemma 11.2.

LEMMA 17.3. There is a constant $c \geq 1$ such that if, for some $n \in \mathbb{N}$,

$$
\left\|\mu^{k}-p_{k}^{H \mu}\right\|_{\infty} \leq A k^{-(D+\gamma) / 2}, \quad 1 \leq k \leq n-1,
$$

then

$$
\left\|\left(\mu^{n}-p_{n}^{H \mu}\right) S_{T}\right\|_{\infty} \leq c\left(1+\frac{A}{\sqrt{T}}\right) n^{-(D+\gamma) / 2} .
$$

The proof of the above lemma, although similar in spirit to the proof of Lemma 11.3 , is technically more complicated. For the case when $\mu$ is symmetric, a proof of the above lemma is given in [2]. We give below an adaptation of that proof.

17.1. Proof of Lemma 17.3. Let $\mu^{\vee n}(x, y)=\mu^{n}(y, x), x, y \in \Gamma$, and let $L_{H \mu} \vee$ be the homogenized sub-Laplacian associated with $\mu^{\vee}$. Note that $L_{H \mu} \vee$ is just the formal adjoint of $L_{H \mu}$ and that its heat kernel $p_{t}^{\vee H \mu}(x, y)$ satisfies $p_{t}^{\vee H \mu}(x, y)=p_{t}^{H \mu}(y, x), x, y \in N$.

Finally, let $\psi^{\vee j}, 1 \leq j \leq n_{2}$, and $\psi^{\vee i j}, 1 \leq i, j \leq n_{1}$, be respectively the first and second order correctors associated with $\mu^{\vee}$. 
We set

$$
W_{t}(x, y)=\sum_{1 \leq j \leq n_{2}} \psi^{j}(x) X_{j}^{x} p_{t}^{H \mu}(x, y)+\sum_{1 \leq i, j \leq n_{1}} \psi^{i j}(x) X_{i}^{x} X_{j}^{x} p_{t}^{H \mu}(x, y)
$$

and

$$
W_{t}^{\vee}(x, y)=\sum_{1 \leq j \leq n_{2}} \psi^{\vee j}(y) X_{j}^{y} p_{t}^{H \mu}(x, y)+\sum_{1 \leq j \leq n_{1}} \psi^{\vee i j}(y) X_{i}^{y} X_{j}^{y} p_{t}^{H \mu}(x, y),
$$

where the superscripts $x$ and $y$ denote differentiation with respect to the $x$ and $y$ variables respectively.

Let

$$
\begin{aligned}
U_{t} & =p_{t}^{H \mu}+W_{t}, \\
U_{t}^{\vee} & =p_{t}^{H \mu}+W_{t}^{\vee} .
\end{aligned}
$$

Let us also fix a $T \geq 1$. Then, to prove (17.5), it is enough to prove that

$$
\left\|\mu^{n} S_{T}-U_{n+T}\right\|_{\infty} \leq c\left(1+\frac{A}{\sqrt{T}}\right) n^{-(D+\gamma) / 2} .
$$

We have

$$
\begin{aligned}
U_{n+T}-\mu^{n} S_{T}= & U_{n+T}-\mu^{n-1} U_{1+T}+\mu^{n-1} U_{1+T}-\mu^{n} S_{T} \\
= & \sum_{0 \leq i \leq n-2}\left(\mu^{i} U_{n-i+T}-\mu^{i+1} U_{n-i-1+T}\right)+\mu^{n-1} U_{1+T}-\mu^{n} S_{T} \\
= & \sum_{0 \leq i \leq n-2} \mu^{i}\left(U_{n-i+T}-\mu U_{n-i-1+T}\right)+\mu^{n-1} U_{1+T}-\mu^{n} S_{T} \\
= & \sum_{0 \leq i \leq n / 2} \mu^{i}\left(U_{n-i+T}-\mu U_{n-i-1+T}\right) \\
& +\sum_{n / 2<i \leq n-2} \mu^{i}\left(U_{n-i+T}-\mu U_{n-i-1+T}\right) \\
& +\mu^{n-1}\left(U_{1+T}-\mu S_{T}\right) \\
= & \sum_{0 \leq i \leq n / 2} \mu^{i}\left(U_{n-i+T}-\mu U_{n-i-1+T}\right) \\
& +\sum_{n / 2<i \leq n-2}\left(\mu^{i}-U_{i}^{\vee}\right)\left(U_{n-i+T}-\mu U_{n-i-1+T}\right) \\
& +\sum_{n / 2<i \leq n-2} U_{i}^{\vee}\left(U_{n-i}-\mu U_{n-i-1+T}\right) \\
& +\left(\mu^{n-1}-U_{n-1}^{\vee}\right)\left(U_{1+T}-\mu S_{T}\right) \\
& +U_{n-1}^{\vee}\left(U_{1+T}-\mu S_{T}\right) .
\end{aligned}
$$


In the rest of the proof, we make repeated use of (14.10) and (6.10). We have

$$
\begin{aligned}
& \sum_{0 \leq i \leq n / 2}\left\|\mu^{i}\left(U_{n-i+T}-\mu U_{n-i-1+T}\right)\right\|_{\infty} \\
& \quad \leq \sum_{0 \leq i \leq n / 2}\left\|\mu^{i}\right\|_{1}\left\|\left(U_{n-i}-\mu U_{n-i-1}\right)\right\|_{\infty} \\
& \leq \sum_{0 \leq i \leq n / 2} c(n-i-1+T)^{-(D+3) / 2} \\
& \leq c n^{-(D+1) / 2} .
\end{aligned}
$$

By the inductive hypothesis (17.4),

$$
\begin{aligned}
& \sum_{n / 2<i \leq n-2}\left\|\left(\mu^{i}-U_{i}^{\vee}\right)\left(U_{n-i+T}-\mu U_{n-i-1+T}\right)\right\|_{\infty} \\
& \quad \leq \sum_{n / 2<i \leq n-2}\left\|\left(\mu^{i}-U_{i}^{\vee}\right)\right\|_{\infty}\left\|\left(U_{n-i+T}-\mu U_{n-i-1+T}\right)\right\|_{1} \\
& \quad \leq \sum_{n / 2<i \leq n-2} A i^{-(D+\gamma) / 2} c(n-i-1+T)^{-3 / 2} \\
& \quad \leq c A \frac{1}{\sqrt{T}} n^{-(D+\gamma) / 2} .
\end{aligned}
$$

We have (arguing as in the proof of Lemma 11.3) that

$$
\left\|\mu S_{T}-p_{T}^{H \mu}\right\|_{1} \leq \frac{c}{\sqrt{T}}, \quad T \geq 1 .
$$

Also, by (6.10),

$$
\left\|p_{T+1}^{H \mu}-p_{T}^{H \mu}\right\|_{1} \leq \frac{c}{T}, \quad T \geq 1
$$

Hence

$$
\begin{aligned}
& \left\|\left(\mu^{n-1}-U_{n-1}^{\vee}\right)\left(U_{1+T}-\mu S_{T}\right)\right\|_{\infty} \\
& \quad \leq\left\|\mu^{n-1}-U_{n-1}^{\vee}\right\|_{\infty}\left\|U_{1+T}-\mu S_{T}\right\|_{1} \leq c A \frac{1}{\sqrt{T}} n^{-(D+\gamma) / 2} .
\end{aligned}
$$

To estimate the remaining term in (17.7), we observe that

$$
\begin{aligned}
& \sum_{n / 2<i \leq n-2} U_{i}^{\vee}\left(U_{n-i+T}-\mu U_{n-i-1+T}\right)+U_{n-1}^{\vee}\left(U_{1+T}-\mu S_{T}\right) \\
& =U_{[n / 2]+1}^{\vee} U_{n-[n / 2]-1+T}-U_{n-1}^{\vee} \mu S_{T} \\
& \quad+\sum_{n / 2<i \leq n-2}\left(U_{i+1}^{\vee}-U_{i}^{\vee} \mu\right) U_{n-i-1+T} .
\end{aligned}
$$


Now

$$
\begin{aligned}
& \sum_{n / 2<i \leq n-2}\left\|\left(U_{i+1}^{\vee}-U_{i}^{\vee} \mu\right) U_{n-i-1+T}\right\|_{\infty} \\
& \quad \leq \sum_{n / 2<i \leq n-2}\left\|U_{i+1}^{\vee}-U_{i}^{\vee} \mu\right\|_{\infty}\left\|U_{n-i-1+T}\right\|_{1} \\
& \quad \leq \sum_{n / 2<i \leq n-1} c i^{-(D+3) / 2} \\
& \quad \leq c n^{-(D+1) / 2} .
\end{aligned}
$$

Also

$$
\begin{aligned}
U_{[n / 2]+1}^{\vee} & U_{n-[n / 2]-1+T}-U_{n-1}^{\vee} \mu S_{T} \\
= & \left(p_{[n / 2]+1}^{H \mu}+W_{[n / 2]+1}^{\vee}\right)\left(p_{n-[n / 2]-1+T}^{H \mu}+W_{n-[n / 2]-1+T}\right) \\
& -\left(p_{n-1}^{H \mu}+W_{n-1}^{\vee}\right) \mu S_{T} \\
= & U_{[n / 2]+1}^{\vee} W_{n-[n / 2]-1+T}+W_{[n / 2]+1}^{\vee} U_{n-[n / 2]-1+T} \\
& +W_{n-1}^{\vee} \mu S_{T}+p_{[n / 2]+1}^{H \mu} p_{n-[n / 2]-1+T}^{H \mu}-p_{n-1}^{H \mu} \mu S_{T} .
\end{aligned}
$$

By (6.10), for all $x, y \in \Gamma$,

$$
\left|p_{n+T}^{H \mu}(x, y)-\sum_{z \in \Gamma} p_{[n / 2]+1}^{H \mu}(x, z) p_{n-[n / 2]-1+T}^{H \mu}(z, y)\right| \leq c n^{-(D+1) / 2}
$$

and

$$
\left|p_{n+T}^{H \mu}(x, y)-\sum_{z \in \Gamma} p_{n-1}^{H \mu}(x, z)\left(m S_{T}\right)(z, y)\right| \leq c n^{-(D+1) / 2} .
$$

Hence

$$
\left\|p_{[n / 2]+1}^{H \mu} p_{n-[n / 2]-1+T}^{H \mu}-p_{n-1}^{H \mu} \mu S_{T}\right\|_{\infty} \leq c n^{-(D+1) / 2} .
$$

It follows that

$$
\begin{aligned}
&\left\|U_{[n / 2]+1}^{\vee} U_{n-[n / 2]-1+T}-U_{n-1}^{\vee} \mu S_{T}\right\|_{\infty} \\
& \leq\left\|U_{[n / 2]+1}^{\vee}\right\|_{1}\left\|W_{n-[n / 2]-1+T}\right\|_{\infty} \\
&+\left\|W_{[n / 2]+1}^{\vee}\right\|_{\infty}\left\|U_{n-[n / 2]-1+T}\right\|_{1}+\left\|W_{n-1}^{\vee}\right\|_{\infty}\left\|\mu S_{T}\right\|_{1} \\
&+\left\|p_{[n / 2]+1}^{H \mu} p_{n-[n / 2]-1+T}^{H \mu}-p_{n-1}^{H \mu} \mu S_{T}\right\|_{\infty} \\
& \leq c(n-[n / 2]-1+T)^{-(D+1) / 2}+c([n / 2]+1)^{-(D+1) / 2} \\
&+c(n-1)^{-(D+1) / 2}+c n^{-(D+1) / 2} \\
& \leq c n^{-(D+1) / 2} .
\end{aligned}
$$


Summing (17.8)-(17.12), we obtain (17.6) and the lemma follows.

18. The corrected monomials $Q_{P_{i}}^{\psi}$. The goal of this section is to construct the corrected monomials $Q_{P_{i}}^{\psi}$ appearing in Theorem 1.11.

Let the monomials $P_{i}, i=0,1,2, \ldots$, be as in Section 6.1 and let us associate with these monomials and the sub-Laplacian $L_{H \mu}$ polynomials $Q_{P_{i}}$ satisfying (6.6).

Note that, by (6.5) and (6.6), we can associate with every polynomial $P(t, x)$ on $\mathbb{R} \times N$ another polynomial $Q(t, x)$ satisfying

$$
\begin{gathered}
\left(\frac{\partial}{\partial t}+L_{H \mu}\right) Q(t, x)=P(t, x), \\
\operatorname{deg}_{H} Q(t, x)=\operatorname{deg}_{H} P(t, x)+2 .
\end{gathered}
$$

Then the corrected monomials $Q_{P_{i}}^{\psi}$ will be furnished by the following:

Proposition 18.1. With every monomial $Q_{P_{i}}(t, g)$ with $\operatorname{deg}_{H} Q_{P_{i}}=d$, as above, we can associate a polynomial

$$
Q_{P_{i}}^{\psi}(t, x)=P_{i}(t, x)+\sum_{0 \leq j \leq v_{d-1}} \psi_{j}^{i}(x) P_{j}(t, x)
$$

satisfying

$$
\left(\frac{\partial}{\partial t}+L_{H \mu}\right) Q_{P_{i}}=\left(\partial_{1}+(I-\mu)\right) Q_{P_{i}}^{\psi}
$$

and where the functions $\psi_{j}^{i}$ are of type $P$.

Before we continue with the proof of Proposition 18.1, let us observe that by (14.10) we can take (1) for $1<i \leq v_{1}$ (note that $v_{1}=n_{1}$ ),

$$
Q_{P_{i}}^{\psi}=P_{i}+\psi^{i}
$$

and (2) for $v_{1}<i \leq v_{2}$,

$$
Q_{P_{i}}^{\psi}=P_{i}+\sum_{1 \leq j \leq n_{2}} \psi^{j} X_{j} P_{i}+\sum_{1 \leq \ell, j \leq n_{1}} \psi^{\ell j}(z) X_{\ell} X_{j} P_{i} .
$$

Proof OF Proposition 18.1. By (18.4) and (18.5) we can assume that $k \geq 3$. Then as a first approximation to $Q_{P_{i}}^{\psi}$ we take

$$
Q_{P_{i}}^{\psi, 1}=Q_{P_{i}}+\sum_{1 \leq j \leq n_{2}} \psi^{j} X_{j} Q_{P_{i}}+\sum_{1 \leq \ell, j \leq n_{1}} \psi^{\ell j} X_{\ell} X_{j} Q_{P_{i}}
$$


By (14.10),

$$
\left(\partial_{1}+(I-\mu)\right) Q_{P_{i}}^{\psi, 1}=\left(\frac{\partial}{\partial t}+L_{H \mu}\right) Q_{P_{i}}+\sum_{0 \leq j \leq v_{d-3}} f_{j}^{i, 1} P_{j},
$$

where the functions $f_{j}^{i, 1}$ are of type P.

Making use of (18.1), for every $v_{d-4}<\ell \leq v_{d-3}$, we consider a polynomial $R_{\ell}$ satisfying

$$
\begin{gathered}
\left(\frac{\partial}{\partial t}+L_{H \mu}\right) R_{\ell}=-P_{\ell}, \\
\operatorname{deg}_{H} Q_{\ell}=\operatorname{deg}_{H} P_{\ell}+2=d-1 .
\end{gathered}
$$

Arguing in the same way as for the definition of the correctors, we consider functions $\phi_{\mu}^{i, 1}$ which are of type $\mathrm{P}$ and which satisfy

$$
(I-\mu) \phi_{\ell}^{i, 1}=-f_{\ell}^{i, 1}+\left\langle f_{\ell}^{i, 1}\right\rangle \quad \text { and } \quad\left\langle\phi_{\ell}^{i, 1}\right\rangle=0 .
$$

Let

$$
R_{\ell}^{f}=\left\langle f_{\ell}^{i, 1}\right\rangle\left(R_{\ell}+\sum_{1 \leq j \leq n_{2}} \psi^{j} X_{j} R_{\ell}+\sum_{1 \leq \lambda, j \leq n_{1}} \psi^{i j} X_{\lambda} X_{j} R_{\ell}\right)+\phi_{\ell}^{i, 1} P_{\ell} .
$$

As a second approximation to $Q_{P_{i}}^{\psi}$ we consider the corrected polynomial

$$
Q_{P_{i}}^{\psi, 2}=Q_{P_{i}}^{\psi, 1}+\sum_{v_{d-4}<\ell \leq v_{d-3}} R_{\ell}^{f}
$$

This polynomial satisfies

$$
\left(\partial_{1}+(I-\mu)\right) Q_{P_{i}}^{\psi, 2}=\left(\frac{\partial}{\partial t}+L_{H \mu}\right) Q_{P_{i}}+\sum_{0 \leq j \leq n_{d-4}} f_{j}^{i, 2} P_{j},
$$

where the functions $f_{j}^{i, 2}$ are again of type $\mathrm{P}$.

We repeat the same procedure another $d-2$ times. The polynomial $Q_{P_{i}}^{\psi, k}$ that we obtain in the end will satisfy (18.2) and (18.3).

19. Harnack inequalities for higher order spatial differences. If $\Gamma$ is not nilpotent, then the analogue of (1.21) for higher order spatial differences is in general false. To see this, let us assume for simplicity that there is a finite subgroup $M \leq \Gamma$ such that $\Gamma=\Gamma_{N} M$ and $\Gamma \cap M=\{e\}$ (i.e., $\Gamma$ is isomorphic to the semidirect product $\Gamma_{N} \lambda M$ ) and let us consider the function

$$
u=P_{j}+\psi^{j},
$$

where $k_{1}<j \leq n_{1}$. This function grows linearly; that is, there is a $c>0$ such that

$$
\sup \left\{|u| ; U^{r}\right\} \leq c r, \quad r \geq 1,
$$


and it satisfies $(I-\mu) u=0$. Also, $\partial_{z} \partial_{w} u=\partial_{z} \partial_{w} \psi^{j}$ for all $z, w \in M$. So, if $\partial_{z} \partial_{w} \psi^{j} \neq 0$, then the inequality

$$
\left.\sup \left\{\left|\partial_{z} \partial_{w} u\right| ; M\right\} \leq c r^{-2} \sup \left\{|u| ; U^{r}\right)\right\}, \quad r \geq 1,
$$

is false.

20. Berry-Esseen estimates for the differences. The goal of this section is to prove the Berry-Esseen estimates (1.28) and (1.30).

We use the notation of Section 17. We set

$$
W_{t}(x, y)=\sum_{1 \leq j \leq n_{2}} \psi^{j}(x) X_{j}^{x} p_{t}^{H \mu}(x, y)+\sum_{1 \leq i, j \leq n_{1}} \psi^{i j}(x) X_{i}^{x} X_{j}^{x} p_{t}^{H \mu}(x, y)
$$

and

$$
U_{t}(x, y)=p_{t}^{H \mu}(x, y)+W_{t}(x, y)
$$

Then, by (1.23),

$$
\left\|\mu^{n}-U_{n}\right\|_{\infty} \leq c n^{-(D+1) / 2}
$$

20.1. Proof of Theorem 1.21. It is enough to prove that there is a constant $c>0$ such that, for all $z \in U$,

$$
\left\|\partial_{z} \mu^{n}-\partial_{z} U_{n}\right\|_{\infty} \leq c n^{-(D+2) / 2} .
$$

We have

$$
\begin{aligned}
U_{n}-\mu^{n} & =\sum_{0 \leq i<[n / 2]} \mu^{i} U_{n-i}-\mu^{i+1} U_{n-i-1}+\mu^{[n / 2]} U_{n-[n / 2]}-\mu^{n} \\
& =\sum_{0 \leq i<[n / 2]} \mu^{i}\left(U_{n-i}-\mu U_{n-i-1}\right)+\mu^{[n / 2]}\left(U_{n-[n / 2]}-\mu^{n-[n / 2]}\right)
\end{aligned}
$$

Hence, by (14.10) and (6.10),

$$
\begin{aligned}
\left\|\partial_{z} \mu^{n}-\partial_{z} U_{n}\right\|_{\infty} \leq & \sum_{0 \leq i<[n / 2]}\left\|\partial_{z} \mu^{i}\right\|_{1}\left\|U_{n-i}-\mu U_{n-i-1}\right\|_{\infty} \\
& +\left\|\partial_{z} \mu^{[n / 2]}\right\|_{1}\left\|U_{n-[n / 2]}-\mu^{n-[n / 2]}\right\|_{\infty} \\
\leq & \sum_{0<i<[n / 2]} c i^{-1 / 2}(n-i-1)^{-(D+3) / 2}+c n^{-1 / 2} n^{-(D+1) / 2} \\
\leq & c n^{-(D+2) 2}
\end{aligned}
$$

which proves (20.2) and the theorem follows. 
20.2. Proof of Theorem 1.22. It is enough to prove that there is a constant $c>0$ such that

$$
\left\|\partial_{1} \mu^{n}-\partial_{1} U_{n}\right\|_{\infty} \leq c n^{-(D+3) / 2}, \quad n \in \mathbb{N} .
$$

We have

$$
\begin{aligned}
\partial_{1} U_{n}-\partial_{1} \mu^{n}= & \sum_{0 \leq i<[n / 2]} \mu^{i} \partial_{1} U_{n-i}-\mu^{i+1} \partial_{1} U_{n-i-1} \\
& +\mu^{[n / 2]} \partial_{1} U_{n-[n / 2]}-\partial_{1} \mu^{[n / 2]} \mu^{n-[n / 2]} \\
= & \sum_{0 \leq i<[n / 2]} \mu^{i}\left(\partial_{1} U_{n-i}-\mu \partial_{1} U_{n-i-1}\right) \\
& +\mu^{[n / 2]}\left(\partial_{1}+(I-\mu)\right) U_{n-[n / 2]}-\mu^{[n / 2]}(I-\mu) U_{n-[n / 2]} \\
& -\left(\partial_{1} \mu^{[n / 2]}\right) \mu^{n-[n / 2]} \\
= & \sum_{0 \leq i<[n / 2]} \mu^{i}\left(\partial_{1}+(I-\mu)\right) \partial_{1} U_{n-i-1} \\
& +\mu^{[n / 2]}\left(\partial_{1}+(I-\mu)\right) U_{n-[n / 2]}+\partial_{1} \mu^{[n / 2]}\left(U_{n-[n / 2]}-\mu^{n-[n / 2]}\right) .
\end{aligned}
$$

Hence, by (14.10) and (6.10),

$$
\begin{aligned}
\left\|\partial_{1} \mu^{n}-\partial_{1} U_{n}\right\|_{\infty} \leq & \sum_{0 \leq i<[n / 2]}\left\|\mu^{i}\right\|_{1}\left\|\left(\partial_{1}+(I-\mu)\right) \partial_{1} U_{n-i-1}\right\|_{\infty} \\
& +\left\|\mu^{[n / 2]}\right\|_{1}\left\|\left(\partial_{1}+(I-\mu)\right) U_{n-[n / 2]}\right\|_{\infty} \\
& +\left\|\partial_{1} \mu^{[n / 2]}\right\|_{1}\left\|U_{n-[n / 2]}-\mu^{n-[n / 2]}\right\|_{\infty} \\
\leq & \sum_{0 \leq i<[n / 2]} c(n-i-1)^{-(D+5) 2} \\
& +c n^{-(D+3) / 2}+c n^{-1} n^{-(D+1) / 2} \\
\leq & c n^{-(D+3) / 2},
\end{aligned}
$$

which proves the theorem.

\section{Riesz transforms.}

21.1. Proof of Theorem 1.25. The kernel $K_{k}$ of the operator $R_{k}$ is given by

$$
K_{k}(x, y)=\sum_{n \geq 0} a_{n} \partial_{z_{1}} \cdots \partial_{z_{k}} \mu^{n}(x, y),
$$

where the $a_{n}$ 's are as in the series $(1-t)^{-1 / 2}=\sum_{n \geq 0} a_{n} t^{n}$. 
By (1.14) and (1.21), $K_{k}(x, y)$ satisfies the standard estimates

$$
\left|K_{k}(x, y)\right| \leq \frac{c}{\left|y^{-1} x\right|_{\Gamma_{N}}^{D}}
$$

and

$$
\nabla_{U}^{x} K_{k}(x, y)+\nabla_{U}^{y} K_{k}(x, y) \leq \frac{c}{\left|y^{-1} x\right|_{\Gamma_{N}}^{D+1}},
$$

where the superindices $x$ and $y$ denote differences with respect to the variables $x$ and $y$ respectively.

So by the Calderon-Zygmund theory (cf. [17, 42]), to prove Theorem 1.25, it is enough to prove that the operator $R_{k}$ is bounded on $L^{2}$. This can be done by an almost orthogonality argument (cf. [43], Chapter 7).

Let us denote by $T_{j}, j \in \mathbb{N}$, the operators with kernel $K_{j}$ given by

$$
K_{j}(x, y)=\sum_{2^{j-1} \leq n<2^{j}} a_{n} \partial_{z_{1}} \cdots \partial_{z_{k}} \mu^{n} .
$$

Then $R_{k}=\partial_{z_{1}} \cdots \partial_{z_{k}}+\sum_{j \geq 1} T_{j}$. Also the kernels $K_{j}(x, y)$ satisfy

$$
\sum_{y \in \Gamma_{N}} K_{j}(x, y)=\sum_{x \in \Gamma_{N}} K_{j}(x, y)=0 .
$$

Furthermore, there is a $c>0$ such that, for all $j \in \mathbb{N}$,

$$
\left\|K_{j}\right\|_{1} \leq \sum_{2^{j-1} \leq n<2^{j}}\left|a_{n}\right|\left\|\partial_{z_{1}} \cdots \partial_{z_{k}} \mu^{n}\right\|_{1} \leq c
$$

Hence,

$$
\sup _{j \in \mathbb{N}}\left\|T_{j}\right\|_{L^{2} \rightarrow L^{2}}<\infty .
$$

Finally, by a straightforward calculation we can see that there is a $c>0$ such that, for all $j \in \mathbb{N}$ and $x \in \Gamma_{N}$,

$$
\begin{gathered}
\sum_{x \in \Gamma_{N}}|x|_{\Gamma_{N}}\left|K_{j}(x, y)\right| \leq c 2^{j / 2}, \\
\sum_{y \in \Gamma_{N}}|y| \Gamma_{\Gamma_{N}}\left|K_{j}(x, y)\right| \leq c 2^{j / 2}, \\
\sum_{y \in \Gamma_{N}}\left|K_{j}(x, y)-K_{j}(e, y)\right| \leq c 2^{-j / 2}|x|_{\Gamma_{N}}, \\
\sum_{x \in \Gamma_{N}}\left|K_{j}(x, e)-K_{j}(x, y)\right| \leq c 2^{-j / 2}|y|_{\Gamma_{N}} .
\end{gathered}
$$

It follows from (21.3), (21.4) and (21.5) that there is a $c>0$ such that

$$
\left\|T_{i} T_{j}^{*}\right\|_{L^{2} \rightarrow L^{2}}+\left\|T_{i}^{*} T_{j}\right\|_{L^{2} \rightarrow L^{2}} \leq c 2^{-|i-j| / 2},
$$

and from this we conclude that $R_{k}$ is bounded on $L^{2}$ (for details we refer the reader to [43], pages 623-625).

The same arguments also apply to the operator $R_{k}^{*}$. 
21.2. Proof of Theorem 1.24. If $\Gamma$ is not nilpotent, then the kernel $K_{z}$ of the Riesz transform $R_{z}$ does not necessarily satisfy the estimate (21.2). So to prove theorem 1.24 we use (1.22) and Theorem 1.25.

More precisely, let us consider the kernels

$$
\begin{gathered}
K_{z}^{H \mu}(x, y)=\sum_{n \geq 0} a_{n} \partial_{z} p_{n}^{H \mu}(x, y), \quad x, y \in \Gamma, \\
K_{j}^{H \mu}(x, y)=\sum_{n \geq 0} a_{n} X_{j} p_{n}^{H \mu}(x, y), \quad 1 \leq j \leq n_{1}, x, y \in \Gamma,
\end{gathered}
$$

and let us denote by $R_{z}^{H \varphi}$ and $R_{j}^{H \mu}$ respectively the associated operators.

Arguing as in the proof of Theorem 1.25 in the previous section, we can prove that the operators $R_{j}^{H \mu}$ are bounded on $L^{p}(\Gamma), 1<p<\infty$, and from $L^{1}(\Gamma)$ to weak- $L^{1}(\Gamma)$.

Also, if $x, y, h \in \Gamma_{N}, 0 \leq i, j, \ell \leq k$ and $z=h g_{\ell}$, then

$$
\begin{aligned}
\partial_{z} p_{t}^{H \mu}\left(x g_{i}, y g_{j}\right) & =p_{t}^{H \mu}\left(x g_{i} h g_{\ell}, y g_{j}\right)-p_{t}^{H \mu}\left(x g_{i}, y g_{j}\right) \\
& =p_{t}^{H \mu}\left(x\left(g_{i} h\right)_{N}, y\right)-p_{t}^{H \mu}(x, y) \\
& =\partial_{\left(g_{i} h\right)_{N}} p_{t}^{H \mu}(x, y) .
\end{aligned}
$$

So, if $w \in N$, then

$$
\partial_{w} \partial_{z} p_{t}^{H \mu}\left(x g_{i}, y g_{j}\right)=\partial_{w} \partial_{\left(g_{i} h\right)_{N}} p_{t}^{H \mu}(x, y)
$$

and hence there is a $c>0$ such that, for all $w \in V$ and $t \geq 1$,

$$
\left|\partial_{w} p_{t}^{H \mu}\left(x g_{i}, y g_{j}\right)\right| \leq c t^{-(D+2) / 2} \exp \left(-\frac{\left|x^{-1} y\right|_{\Gamma}^{2}}{c t}\right) .
$$

It follows that

$$
\left|\partial_{w} K_{z}^{H \mu}\left(x g_{i}, y g_{j}\right)\right| \leq \frac{c}{\left|x^{-1} y\right|_{\Gamma}^{D+1}} .
$$

So the operator $R_{z}^{H \mu}$ is also bounded on $L^{p}(\Gamma), 1<p<\infty$, and from $L^{1}(\Gamma)$ to weak- $L^{1}(\Gamma)$.

Let us consider the kernel $K_{z}^{H \mu}(x, y)$ that satisfies the estimate

$$
S_{z}(x, y)=K_{z}(x, y)-K_{z}^{H \mu}(x, y)-\sum_{1 \leq j \leq n_{1}}\left(\partial_{z} \psi^{j}(x)\right) K_{j}^{H \mu}(x, y), \quad x, y \in \Gamma .
$$

Then it follows from (1.31) that for all $\varepsilon \in(0,1)$ there is a $c>0$ such that

$$
|S(x, y)| \leq \frac{c}{\left|x^{-1} y\right|_{\Gamma}^{D+\varepsilon}}, \quad x, y \in G ;
$$


that is, the kernel $S(x, y)$ is integrable and hence the operator

$$
S=R_{z}-R_{z}^{H \varphi}-\sum_{1 \leq j \leq n_{1}}\left(\partial_{z} \psi^{j}\right) R_{j}^{H \mu}
$$

is bounded on $L^{p}(\Gamma), 1 \leq p \leq \infty$.

Hence $R_{z}$ is bounded on $L^{p}, 1<p<\infty$, and from $L^{1}(\Gamma)$ to weak- $L^{1}(\Gamma)$.

The same arguments also apply to the operator $R_{z}^{*}$.

\section{REFERENCES}

[1] Alexopoulos, G. K. (1989). Quelques propriétés des fonctions harmoniques sur les groupes de Lie à croissance polynômiale. C. R. Acad. Sci. Paris Sér. I Math. 308 337-338.

[2] Alexopoulos, G. K. (1990). Convolution powers on discrete groups of polynomial volume growth. In Harmonic Analysis and Number Theory: Papers in Honor of Carl S. Herz 21 (S. W. Drury and M. Ram Murty, eds.) 31-57. Amer. Math. Soc., Providence, RI.

[3] Alexopoulos, G. K. (1992). An application of homogenisation theory to harmonic analysis: Harnack inequalities and Riesz transforms on Lie groups of polynomial growth. Canad. J. Math. 44 1-37.

[4] Alexopoulos, G. K. (1993). An application of Homogenization theory to Harmonic analysis on solvable Lie groups of polynomial growth. Pacific J. Math. 159 19-45.

[5] Alexopoulos, G. K. (1997). Puissances de convolution sur les groupes à croissance polynômiale du volume. C. R. Acad. Sci. Paris Sér. I Math. 324 771-776.

[6] Alexopoulos, G. K. (1998). Sous-laplaciens et densités centrées sur les groupes de Lie à croissance polynômiale du volume. C. R. Acad. Sci. Paris Sér. I Math. 326 539-542.

[7] Alexopoulos, G. K. (2002). Centered Sub-Laplacians with drift on Lie groups of polynomial volume growth. Mem. Amer. Math. Soc. 155 Number 739.

[8] Alexopoulos, G. K. and Lohoué, N. (1993). Sobolev inequalities and harmonic functions of polynomial growth. J. London Math. Soc. 48 452-464.

[9] Avellaneda, M. and Lin, F. H. (1987). Compactness methods in the theory of Homogenization. Comm. Pure Appl. Math. 40 803-847.

[10] Avellaneda, M. and Lin, F. H. (1989). Un théorème de Liouville pour des equations elliptiques avec coefficients périodiques. C. R. Acad. Sci. Paris Sér. I Math. 609 245-250.

[11] BASS, H. (1972). The degree of polynomial growth of finitely generated nilpotent groups. Proc. London Math. Soc. 25 603-614.

[12] Baumslag, G. (1971). Lecture Notes on Nilpotent Groups. Amer. Math. Soc., Providence, RI.

[13] Bensoussan, A., Lions, J. L. and Papanicolaou, G. (1978). Asymptotic Analysis of Periodic Structures. North-Holland, Amsterdam.

[14] Bergström, H. (1969). On the Central Limit Theorem in $\mathbb{R}^{k}$. Z. Wahrsch. Verw. Gebiete 14 $113-126$.

[15] Christ, M. (1995). Temporal regularity of random walks on discrete nilpotent groups. In Proceedings of the Conference in Honor of J.-P. Kahane 141-151. CRC Press, Boca Raton, FL.

[16] Christ, M. and Geller, D. (1984). Singular integral characterizations of Hardy spaces on homogeneous groups. Duke Math. J. 51 547-598.

[17] Coifman, R. and Weiss, G. (1971). Analyse non-commutative sur certains espaces homogènes. Lecture Notes in Math. 242. Springer, New York.

[18] Colding, T. H. and Minicozzi, W. P., II (1997). Harmonic functions with polynomial growth. J. Differential Geom. 46 1-77. 
[19] Crépel, P. and Raugi, A. (1978). Théorème central limite sur les groupes nilpotents. Ann. Inst. H. Poincaré Probab. Statist. 14 145-164.

[20] FELLER, W. (1971). An Introduction to the Theory of Probability and Its Applications 2. Wiley, New York.

[21] Folland, G. B. and Stein, E. (1982). Hardy Spaces on Homogeneous Groups. Princeton Univ. Press.

[22] Gromov, M. (1981). Groups of polynomial growth and expanding maps. Inst. Hautes Études Sci. Publ. Math. 53 53-78.

[23] Hebisch, W. and Saloff-Coste, L. (1993). Gaussian estimates for Markov chains and random walks on groups. Ann. Probab. 21 673-709.

[24] Hörmander, L. H. (1967). Hypoelliptic second order differential operators. Acta. Math. 16 $147-171$.

[25] JaCOBSON, N. (1962). Lie Algebras. Wiley, New York.

[26] Jikov, V. V., Kozlov, S. M. and Oleinik, O. A. (1994). Homogenization of Differential Operators and Integral Functionals. Springer, New York.

[27] Kenig, C. E. (1994). Harmonic Analysis Techniques for Second Order Elliptic Boundary Value Problems. Amer. Math. Soc., Providence, RI.

[28] Kozlov, S. M. (1980). Asymptotics of fundamental solutions for second order differential equations. Mat. Sb. 113 302-323. [English translation (1982) Math. USSR.-Sb. 41 249-267.]

[29] Krylov, N. V. and SAfonov, M. V. (1981). A certain property of solutions of parabolic equations with measurable coefficients. Math. USSR-Izv. 16 151-164.

[30] LAWLER, G. F. (1991). Estimates for differences and Harnack inequality for difference operators coming from random walks with symmetric, spacially inhomogeneous, increments. Proc. London Math. Soc. 63 552-568.

[31] Lohoué, N. and VARopoulos, N. TH. (1985). Remarques sur les transformés de Riesz sur les groupes de Lie nilpotents. C. R. Acad. Sci. Paris Sér. I Math. 30111.

[32] Moustapha, S. and Varopoulos, N. Th. Cambridge Univer. Press. To appear.

[33] NAgEl, A., RiCCI, F and Stein, E. (1990). Harmonic Analysis and fundamental solutions on nilpotent Lie groups. In Analysis and Partial Differential Equations: A Collection of Papers Dedicated to M. Cotlar (C. Sadosky ed.) 249-275. Dekker, New York.

[34] Petrov, V. V. (1975). Sums of Independent Random Variables. Springer, New York.

[35] Raghunathan, M. S. (1972). Discrete Subgroups of Lie Groups. Springer, New York.

[36] Raugi, A. (1978). Théorème de la limite centrale sur les groupes de Lie nilpotents. Z. Wahrsch. Verw. Gebiete 43 149-172.

[37] Raugi, A. (1978). Théorème de la limite centrale pour un produit semidirect d'un groupe de Lie résoluble simplement connexe de type rigide par un groupe compact. Probability Measures on Groups. Lecture Notes in Math. 706 257-324. Springer, New York.

[38] Riesz, F. and VON Sz. NAGY, B. (1968). Leçons d'analyse fonctionelle, 5th ed. GauthierVillars, Paris.

[39] SAFONOV, M. V. (1983). Harnack's inequality for elliptic equations and the Hölder property of their solutions. J. Soviet Math. 21 851-863.

[40] SAloff-Coste, L. (1990). Analyse sur les groupes de Lie à croissance polynômiale. Ark. Mat. 28 315-331.

[41] Sazonov, V. V. (1981). Normal Approximation-Some Recent Advances. Lecture Notes in Math. 879. Springer, New York.

[42] Stein, E. (1970). Singular Integrals and Differentiability Properties of Functions. Princeton Univ. Press.

[43] Stein, E. (1993). Harmonic Analysis: Real-Variable Methods, Orthogonality and Oscillatory Integrals. Princeton Univ. Press. 
[44] STroock, D. W. (1988). Diffusion semigroups corresponding to uniformly elliptic divergence form operators. Séminaire de probabilités XXII. Lecture Notes in Math. 1321 316-347. Springer, New York.

[45] VARADARAJAN, V. S. (1984). Lie Groups, Lie Algebras and Their Representations. Springer, New York.

[46] Varopoulos, N. Th. (1986). Analysis on nilpotent groups. J. Funct. Anal. 66 406-431.

[47] Varopoulos, N. TH. (1987). Fonctions harmoniques positives sur les groupes de Lie. C. $R$. Acad. Sci. Paris Sér. I Math. 304 519-521.

[48] Varopoulos, N. Th. (1988). Analysis on Lie groups. J. Funct. Anal. 76 346-410.

[49] Varopoulos, N. TH. (1994). Wiener-Hopf theory and nonunimodular groups. J. Funct. Anal. 120 467-483.

[50] Varopoulos, N. Th., Saloff-Coste, L. and Coulhon, Th. (1993). Analysis and Geometry on Groups. Cambridge Univ. Press.

[51] Zhikov, V. V. (1989). Spectral approach to asymptotic diffusion problems. Differensial'nye Uravneniya 25 44-50. [English translation (1989) Differential Equations 25 33-39.]

MATHÉMATIQUeS

UNIVERSITÉ DE PARIS-SUD

BÂT. 425

91405 ORSAY CEDEX

FRANCE 